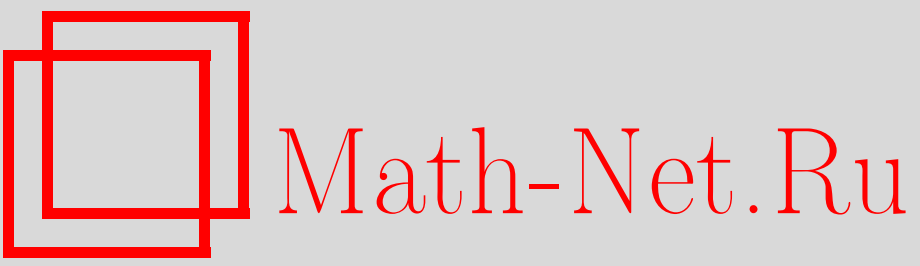

П. Деджованни, Р. Мелин, К. Шобет, Применение методов конформной теории поля в одномерных бесщелевых фермионных системах и их применение к краевым возмущениям квантовых последовательностей Холла с $\nu=1 /(2 p+1)$, ТМФ, 1998, том 117, номер 1, 5-91

DOI: https://doi.org/10.4213/tmf918

Использование Общероссийского математического портала Math-Net.Ru подразумевает, что вы прочитали и согласны с пользовательским соглашением http: //www . mathnet.ru/rus/agreement

Параметры загрузки:

IP : 3.85 .73 .92

26 апреля 2023 г., 13:59:31 


\section{ПРИМЕНЕНИЕ МЕТОДОВ КОНФОРМНОЙ ТЕОРИИ ПОЛЯ В ОДНОМЕРНЫХ БЕСЩЕЛЕВЫХ ФЕРМИОННЫХ СИСТЕМАХ И ИХ ПРИМЕНЕНИЕ К КРАЕВЫМ ВОЗМУЩЕНИЯМ КВАНТОВЫХ ПОСЛЕДОВАТЕЛЬНОСТЕЙ ХОЛЛА С $\nu=1 /(2 p+1)$}

Мы исследуем эффективную конформную теорию поля (КТП), описывающую низкоэнергетические возмущения газа бесспиновых взаимодействующих фермионов на окружности в режиме отсутствия массовой щели (жидкость Люттингера). Мы используем технику функционального интегрирования и свойства модулярных преобразований для вычисления всех корреляционных функций при конечных размерах и конечной температуре. Параметр беспорядка при рассеянии вперед вычислен точно. Мы рассматриваем в рамках нашего подхода к КТП эксперименты Лавлина по переносу заряда в квантовой жидкости Холла на цилиндре. Краевые возмущения, возникающие на фоне заданных возмущений в слое вешества, описываются твистованным аналогом эффективной теории Люттингера. КТП Люттингера, отвечающие заполняющим фракциям с $\nu=1 /(2 p+1)$, оказываются рациональными КТП (РКТП). Генераторы алгебры расширенной симметрии при этом оказываются операторами рождения и уничтожения граничных фермионов и тем самым придают физический смысл описанию граничных возмущений квантовых последовательностей Холла в рамках РКТП.

\section{СОДЕРЖКАНИЕ}

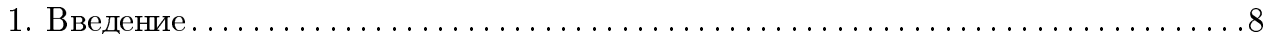

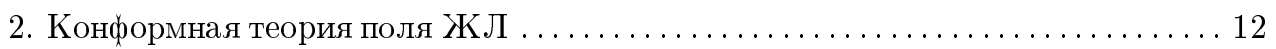

2.1. Модель Люттингера . . . . . . . . . . . . . . . . . . . . . . . . . . . . 12

2.1.1. Свободная теория . . . . . . . . . . . . . . . . . . . . . . 13

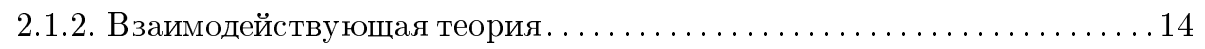

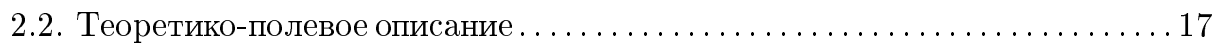

\footnotetext{
${ }^{*}$ Laboratoire de Physique Théorique ENSLAPP, Lyon, France.

E-mail: Pascal.Degiovanni@ens-lyon.fr

${ }^{\dagger}$ Centre de Recherche sur les Très Basses Températures, Grenoble, France. E-mail: melin@crtbt.polycnrs-gre.fr

${ }^{\ddagger}$ Université des Sciences et Techniques du Languedoc, Montpellier, France. E-mail: cris@pollux.ges.fr
} 


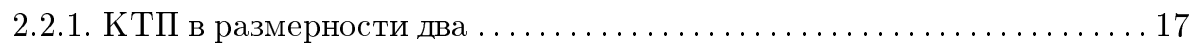

2.2.2. Свободные фермионы на торе . . . . . . . . . . . . . . . . . . 18

2.2.3. Безмассовая модель Тирринга. . . . . . . . . . . . . . . . . . . . . . 19

2.2.4. Явные вычисления в операторном формализме . . . . . . . . . . . . 20

2.3. Бозонное описание эффективной теории Люттингера . . . . . . . . . . 22

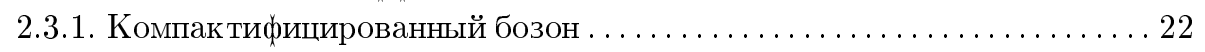

2.3.2. Отождествление ЖЛ с компактифицированными бозонами . . . . . . . 23

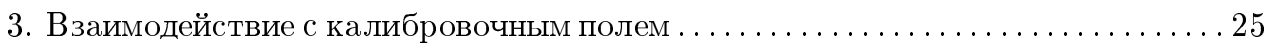

3.1. Компактифищированный бозон, взаимодействующий с постоянным кали-

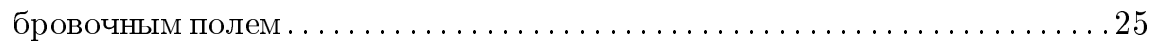

3.2. Калибровочные преобразования . . . . . . . . . . . . . . . . . . 26

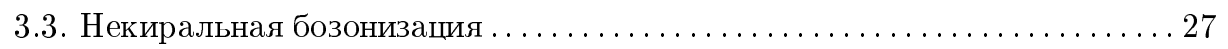

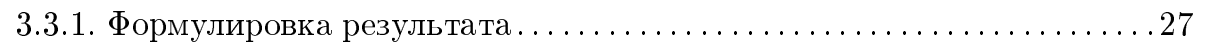

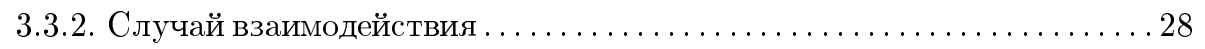

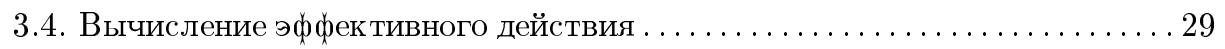

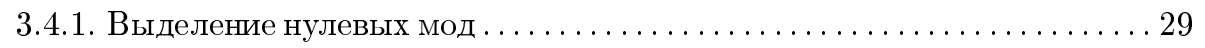

3.4.2. Явное вычисление . . . . . . . . . . . . . . . . . . . . . . 29

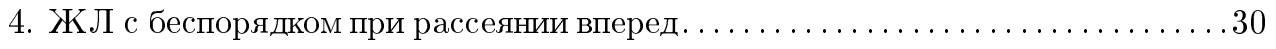

4.1. Общая постановка задачи и обозначения . . . . . . . . . . . . . . 31

4.2. Средние по беспорядку от корреляционных функций . . . . . . . . . . 32

4.3. Средняя свободная энергия, теплоемкость . . . . . . . . . . . . . 33

5. Корреляционные функции заряд-заряд и ток-ток . . . . . . . . . . . . 34

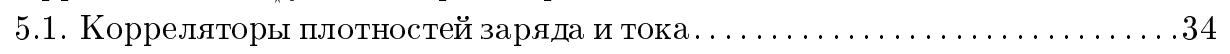

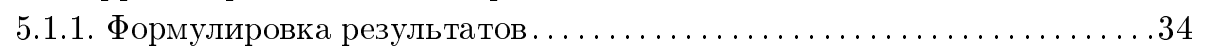

5.1 .2 . Детали вычисления . . . . . . . . . . . . . . . . . . . . . . 35

5.2 Восприимчивость плотности к внешнему потенциалу $\ldots \ldots \ldots \ldots \ldots \ldots 36$

5.2.1. Изменения плотности, вызванные внешним потенциалом . . . . . . . . 36

5.2 .2 . Корреляционные функции . . . . . . . . . . . . . . . . . . . 37

5.3. Постоянные токи в кольце Люттингера . . . . . . . . . . . . . . . 37

6. Корреляционные функции вершинных операторов . . . . . . . . . . . . . 39

6.1. Вершинные операторы . . . . . . . . . . . . . . . . . . . . . . 39

6.2. Корреляционные функции на сфере ...................... 40

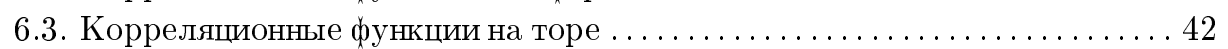

6.4. Свободные фермионы . . . . . . . . . . . . . . . . . . . . . . . . 43

6.5. Учет модельного беспорядка . . . . . . . . . . . . . . . . . . . . . . 45

7. Эксперименты Лавлина и ЖЛ . . . . . . . . . . . . . . . . . . 46

7.1. Плотности физических заряда и тока в ЖЛ ................ 46

7.2. Перетекание заряда между различными киральностями в ЖЛ . . . . . 47

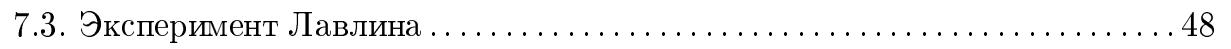

7.4. Другой эксперимент по переносу заряда ..................... 49

8. Рациональные КТП Люттингера............................... 51

8.1. Критерий рациональности для КТП Люттингера.................. 51

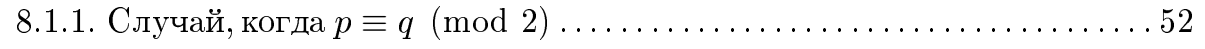

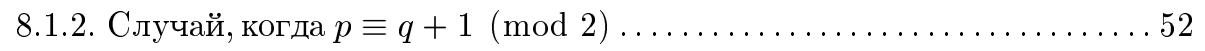


8.2. Расширенные характеры . . . . . . . . . . . . . . . . . . 52

8.3. Статистические суммы рациональных КТП Люттингера . . . . . . . . . . 54

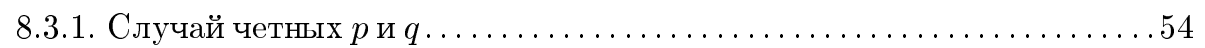

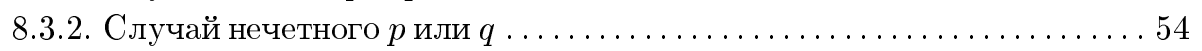

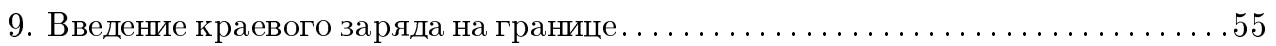

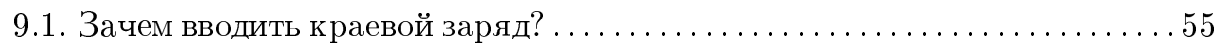

9.2. Статистические суммы с краевым зарядом . . . . . . . . . . . . . . 56

9.2.1. Вычисление статистической суммы . . . . . . . . . . . . . 56

9.2 .2 . Выражение через обобщенные характеры . . . . . . . . . . . . . 57

9.3. Правила слияния в рациональных моделях Люттингера.............. 58

9.4. Дуальность в КТП Люттингера . . . . . . . . . . . . . . . . 60

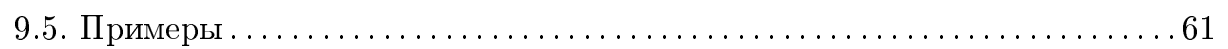

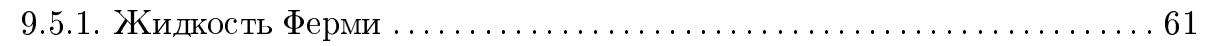

9.5.2. Лавлиновская жидкость Холла в точке общего положения . . . . . . . 62

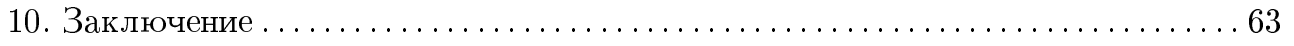

Приложение А. Гамильтонианы в инфракрасном пределе . . . . . . . . . . 64

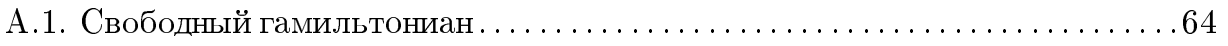

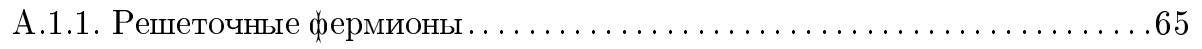

А.1.2. Нерелятивистские фермионы . . . . . . . . . . . . . . . . . 65

А.2. Решеточная модель со взаимодействиями $g_{2}$ и $g_{4} \ldots \ldots \ldots \ldots \ldots \ldots \ldots 6$

Приложение В. Явные вычисления в теории Дирака . . . . . . . . . . . . . 67

В.1. Антипериодические фермионы, взаимодействуюшие с магнитным полем и

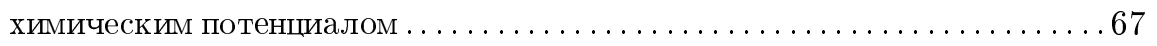

В.1.1. Операторные вычисления с фермионами . . . . . . . . . . . . . . 67

В.1.2. Сравнение фермионных и бозонных результатов в секторе $\mathrm{A}, \mathrm{A} \ldots \ldots 68$

В.1.3. Сектор АР . . . . . . . . . . . . . . . . . . . . . . . . . 68

В.2. Сдвиги и свойства модулярной инвариантности . . . . . . . . . . . . 68

В.2.1. Влияние сдвигов на бозонные статистические суммы . . . . . . . . . . 69

В.2.2. Свойства совместности . ........................ 69

В.3. Секторы РА и РР . . . . . . . . . . . . . . . . . . . . . . . . 71

Приложение С. Условия нормировки для бозонных функциональных интегралов 73

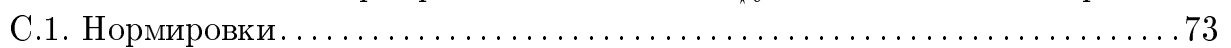

С.2. Вклад фллуктуаций . . . . . . . . . . . . . . . . . . . . . . . . 74

Приложение D. Явные вычисления в бозонной теории . . . . . . . . . . . . . 74

D.1. Инстантоны . . . . . . . . . . . . . . . . . . . . . . . . . . 75

D.2. Вычисление статистической суммы . . . . . . . . . . . . . . . . 75

D.3. Введение калибровочного поля и инстантонный вклад . . . . . . . . . . . .76

Приложение Е. Эллиптические и тета-функции $\ldots \ldots \ldots \ldots \ldots \ldots \ldots \ldots \ldots \ldots \ldots$

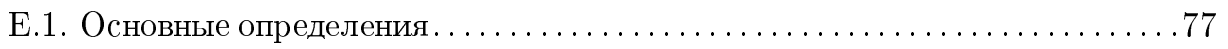

Е.2. Соотношения между эллиптическими и тета-функциями и обратный лап-

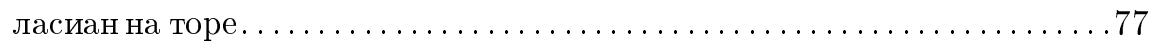

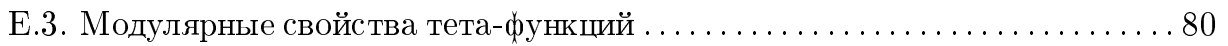

Приложение F. Нерелятивистские фермионы $\ldots \ldots \ldots \ldots \ldots \ldots \ldots \ldots \ldots \ldots \ldots \ldots$

F.1. Отклик на внешний потенциал $\ldots \ldots \ldots \ldots \ldots \ldots \ldots \ldots \ldots \ldots \ldots \ldots \ldots \ldots \ldots \ldots \ldots \ldots$ 
F.2. Корреляции плотность-плотность . . . . . . . . . . . . . . . . 82

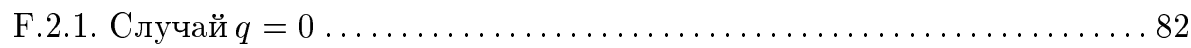

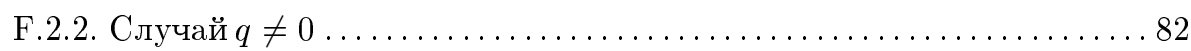

Приложение G. Операторные вычисления и сравнение с результатами КТП . . . 83

Приложение Н. Дуальность для свободного бозона . . . . . . . . . . . . . . 85

Н.1. Введение вспомогательных полей . . . . . . . . . . . . . . . . . 85

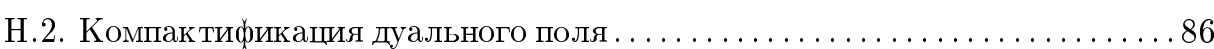

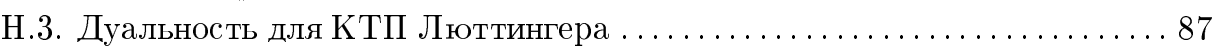

H.4. Тождества Уорда в дуальной теории ..................... 88

\section{1. ВВЕДЕНИЕ}

Проблема многих тел в физике конденсированных сред была предметом интенсивных исследований в течение многих десятилетий. Первые теории такого вида относятся к 1956 г., когда была построена теория жидкости Ландау-Ферми. Эта теория дает достаточно полное феноменологическое описание трехмерных нормальных систем взаимодействуюших фермионов $[1,2]$. В этой теории основную роль играет понятие квазичастичных возмущений, находящихся благодаря принципу адиабатического продолжения [3], во взаимно однозначном соответствии с возмушениями невзаимодействуюшего газа. Теория жидкости Ландау-Ферми описывает многочастичное состояние с помощью нескольких феноменологических параметров (эффективной массы и параметров Ландау), которые являются экспериментально измеряемыми величинами, такими как удельная теплоемкость, сжимаемость, магнитная восприимчивость и скорость звука. Подробное изложение этой теории можно найти в многочисленных книгах (см., например, $[3,4])$. Микроскопическое описание феноменологической теория Ландау было дано с использованием методов квантовой теории поля [4-6]. Адиабатическое включение взаимодействия [3] требует, чтобы обратное время жизни частицы $1 / \tau(\mathbf{k})$ было много меньше, чем энергия квазичастицы $\epsilon(\mathbf{k})$; тогда мы можем легко выбрать скорость включения взаимодействия $R$, для которой

$$
\frac{1}{\tau(\mathbf{k})} \ll R \ll \epsilon(\mathbf{k}) .
$$

В трехмерном случае время жизни квазичастицы может быть вычислено с помощью "золотого правила" [3]: $\tau(\mathbf{k}) \sim \epsilon^{-2}(\mathbf{k})$. Отсюда условие (1.1) вьполняется при произвольно малых энергиях. Это означает, что квазичастица не распадается в течение времени включения взаимодействия и что скорость включения этого взаимодействия должна отвечать соотношениям неопределенности Гейзенберга. Данное условие нарушается в одномерных (1D) системах; это говорит о том, что взаимодействующие одномерные фермионные системы не принадлежат к классу ферми-жидкостей.

Невозможность описания одномерных взаимодействующих фермионов с точки зрения ферми-жидкости было впервые доказано Дзялошинским [7], который показал, что в такой одномерной системе отсутствует поверхность Ферми, поскольку исчезает вычет квазичастицы, а фермионные числа заполнения подчиняются степенному закону 
распределения и не содержат разрьва. Другие специфические свойства взаимодействующих 1D-фермионных систем были получены позднее. Например, в системе с локальными взаимодействиями функция Грина обрашается в нуль [8] в точке, в которой происходит (катастрофическое) нарушение ортогональности [9]. В остальных точках корреляции убывают алгебраически и задаются аномальными критическими экспонентами $[8,10-12]$. В жидкости спина $1 / 2$ также происходит разделение спина и заряда [13]: дополнительный электрон, возникаюший в этой системе, расшепляется в спиновый и зарядовый пакеты, движушиеся с разными скоростями. По поводу соотношений между ферми-жидкостью и не ферми-жидкостью можно обратиться к работе [14], см. также библиографию, приведенную в ней.

Первые шаги на пути построения точно решаемых 1D-фермионных систем были сделаны Томонагой [15], Люттингером [16] и Маттисом и Лейбом [17]. Технически использовалась линеаризация свободноэлектронного дисперсионного соотношения вблизи двух точек Ферми, которая задает отображение в двумерную теорию Дирака со взаимодействием (безмассовая модель Тирринга) и может быть в дальнейшем бозонизована. Халдейн [8] показал, что такие низкоэнергетические свойства присуши широкому классу 1D-систем, например фермионным 1D-жидкостям или некоторым безмассовым фазам цепочек со спином $1 / 2$. На самом деле, можно отождествить низкоэнергетическое поведение некоторых моделей с поведением жидкости Люттингера (ЖЛ). Например, это было сделано в [18] для цепочки Гейзенберга спина $1 / 2$, которая преобразовывалась в фермионную жидкость с помошью преобразования Йордана-Вигнера, а в [19] для 1D-модели Хаббарда. В этом смысле ЖЛ аналогична 3D-теории Ландау, поскольку она задает точно решаемую универсальную эффективную низкоэнергетическую теорию с помошью нескольких параметров.

Другое объединяюшее описание одномерных квантовых систем дается двумерными конформными теориями поля (КТП). Эти теории первоначально были тшательно изучены в рамках подхода к эффективным теориям поля при наличии двумерных критических точкек [20], но они также оказались полезными и при исследовании одномерных квантовых систем. Это не что иное, как эквивалентность между $D$-мерными квантовыми системами при конечной температуре и подходящими классическими системами в размерности $D+1$ [21]. Разумеется, поскольку КТП на самом деле описывают “безмассовые" теории поля, они также годятся для описания одномерных бесщелевых систем. Тем самым исходя из этих общих соображений мы можем ожидать, что ЖЛ также допускает подобное эффективное низкоэнергетическое описание.

Имеются многочисленные обзоры по КТП. Мы рекомендуем заинтересованному читателю оригинальные статьи [20] о КТП на плоскости и [22] о КТП на торе, а также многочисленные обзоры, например лекции [23] и [24], книгу Итцыксона и Друффе [25, гл. 9] или более позднюю книгу Кетова [26]. С точки зрения данной статьи особенно сушественна работа [27], где наиболее подробно исследован случай $c=1 \mathrm{KTП.} \mathrm{Разумеется,}$ эта библиография по КТП далеко не полна, но может послужить хорошей отправной точкой для первого знакомства с этой областью.

В данной статье мы исследуем соотношения между ЖЛ и КТП. Более точно, мы отождествляем бесспиновую ЖЛ конечного размера и при конечной температуре, но без UMKLAPP-членов с теорией поля компактифицированного бозона на двумерном 
торе с нужным топологическим весом. Напомним, что, поскольку рассматриваются $1 \mathrm{D}$-квантовые жидкости, наиболее полная модель включает в себя полную $g$-топологию. В работе [28] показано, что приблизительньй анализ скейлингового режима (первоначально примененньй Андерсоном к решению проблемы Кондо [29]) дает условие сушествования области взаимодействий, в которой в фиксированной точке остаются только взаимодействия $\left(g_{2}, g_{4}\right)$. В данной работе мы ограничиваем рассмотрение именно таким случаем, когда имеются лишь два взаимодействия $g_{2}$ и $g_{4}$. Эти взаимодействия определяют эффективные параметры теории в смысле ренормгруппы, подобно тому как параметры Ландау определяют свойства трехмерной взаимодействующей ферми-системы [30]. Мы увидим, как эффективная КТП для ЖЛ изменяется при варьировании параметров $g_{2,4}$.

Отождествление ЖЛ и теории компактифицированных бозонов было предложено в [31] для квантовых спиновых цепочек, и в конще восьмидесятых годов специалисты в теории струн также исследовали соответствие между теорией Дирака и компактифицированными бозонами, но только в модулярно-инвариантных секторах [32]. Напротив, мы будем рассматривать такой специфический сектор теории фермионных ЖЛ, который с очевидностью не будет модулярно-инвариантен. Тем самым формулы бозонизации должны учитывать граничные условия, а потому нам понадобится топологический член. Для наглядности все нужные формулы будут выводиться достаточно простым образом. Тем не менее они уже возникали в литературе (см. $[33,34])$, но нигде не применялись к описанию одномерных систем конденсированной материи. И все же нам удалось обнаружить несколько не очень известных, к сожалению, статей, имеюших явное отношение к этому тонкому вопросу: в работе [35] Ву и Ю описали КТП Люттингера, исследуя идеальный газ эксклюзонов на основе идей Халдейна [36] относительно описания обобшенной статистики. В работе [37] КТП Люттингера была получена исходя из свойств локальности алгебры операторных произведений и были отмечены присушие ей модулярные свойства и свойства дуальности. В этой работе также обстоятельно обсуждается связь между моделью Тирринга и моделью синус-Гордон. Статья [37] может дополнить нашу работу.

Возврашаясь к формальным основам метода, отметим, что объемлющий обзор бозонизации был дан в статье [38] (см. также работу Жоликера и Ле Гюлу [39]), в которой также использовались функциональные методы в термодинамическом пределе и пределе нулевой температуры, т.е. на плоскости. Упомянем также классические работы Коулмена [40] и Мандельштама [41], в которых был развит операторный подход к бозонизашии массивной модели Тирринга.

В завершение этого короткого обзора подходов к бозонизации ЖЛ отметим работу [42], в которой квантовые XXZ-цепочки спина $1 / 2$, находяшиеся в состоянии, далеком от наполовину заполненного, и живущие на окружности с периодическими или открытыми граничными условиями для спина, были подвергнуты численному анализу. В соответствии с утверждением авторов этой статьи предсказания КТП для спектра согласуются с численными оценками, основанными на методе ренормгруппы, примененном $\mathrm{k}$ матрице плотности.

В данной статье мы явно покажем, что все те физические величины ЖЛ, которые могут быть вычислены в подходе к бозонизации, использованном Халдейном в [8], могут 
также быть явно вычислены методом функционального интегрирования. Единственное дополнительное ограничение состоит в том, что взаимодействие должно быть локальным, чтобы конформная инвариантность имела место. Как показано в работе [43], нелокальные взаимодействия разрушают жидкость Ферми, т.к. теряется когерентность квазичастиц и они не сохраняют конформную инвариантность из-за своей конечной протяженности. Однако на достаточно больших расстояниях все несингулярные взаимодействия могут считаться локальными.

Одним из мотивов развития функциональной бозонизации ЖЛ при конечных размерах исследуемых систем явилась необходимость обрашения с внешним магнитным полем и неупорядоченным рассеянием вперед (случайный потенциал вместе с длинноволновыми фолуктуациями плотности). Проблема неупорядоченной ЖЛ рассматривалась в работе [44] с точки зрения теории перенормировок. По сравнению с предлагаемой работой модель в работе [44] включает в рассмотрение рассеяние назади отвечает локализованной фазе. Однако способом работы [44] невозможно осуществить прямое вычисление корреляционных функций с помощью функционального интеграла. Рассеяние вперед не приводит к локализации задачи, но в этом подходе средние по ансамблю могут быть явно вычислены для всех произведений корреляционных функций.

Формулировка ЖЛ в терминах КТП удивительным образом обеспечивает весьма простое описание эффективной теории краевых возмушений в жидкости с дробным квантовым эффектом Холла (ДКЭХ). Первым, кто указал на то, что краевые возмущения ДКЭХ-жидкости описываются ЖЛ, был Вен $[45,46]$. Это соотношение было глубоко исследовано для краевых состояний ДКЭХ-жидкости, имеющей форму диска (в этом случае на границе возникает киральная ЖЛ). В работе [47] Халдейн и Резай численно исследовали цилиндрические геометрии, которые могут порождать ДКЭХ-жидкость. Поэтому интересно найти точное соответствие между теорией поля краевых возмущений на цилиндре с ЖЛ в соответствии с рассуждениями Гальперина [48], Вена и представителей сообшества специалистов по КТП - Капелли и др. [49]. Здесь мы утверждаем, что краевым состояниям ДКЭХ-жидкости на цилиндре соответствуют некиральные ЖЛ, исследуемые в данной работе (см. статью Лавлина [50]). Более точно, КТП Люттингера, соответствуюшие заполняюшей фракции $\nu=1 /(2 p+1)$, суть рациональные конформные теории поля (РКТП) [51]. Их статистические суммы совпадают со статистическими суммами, полученными Капелли и Зембой [52]. Эти авторы получили свой результат исходя из свойств модулярной инвариантности, мы же найдем ответ из одномерной эффективной теории взаимодействующих фермионов. Разумеется, в ДКЭХ-жидкостях эти взаимодействия индуцируются сильными корреляциями электронов в жидкости Холла. Мы продолжаем этот анализ, вводя краевой заряд на границе для описания башни (потомков) краевых возмушений над заданным возмушением в слое. Статистическая сумма такой “твистованной” КТП Люттингера должна отражать структуру возмушений над заданными возмушениями в слое. Следуя Фишеру и Стоуну [53], также интересно понять, какие краевые возмущения соответствуют введению физического электрона на одном или двух краях цилиндра. Соответствующие конформные поля порождают расширенную алгебру максимальных симметрий для КТП Люттингера, тем самым обеспечивая наиболее простое описание возмушений ДКЭХ-жидкости. 
План предлагаемой работы следующий: в разделе 2 мы приводим основные факты из статьи Халдейна в применении к безмассовой модели Тирринга на торе. В разделе 3 исследуется взаимодействие с калибровочным полем и приводится доказательство нашей формулы бозонизации посредством функционального метода. В разделе 4 явно исследуется рассеяние вперед. В разделах 5 и 6 вычисляются корреляционные функции. Сначала вычисляются плотности заряда и тока, а также зарядовая и токовая восприимчивости по отношению к внешнему электрическому потенциалу и к магнитному потоку. Затем вычисляются корреляционные функции всех вершинных операторов. Среди них имеются фермионные операторы Люттингера.

Завершив изучение обшей КТП Люттингера, мы покажем, как некоторые из этих КТП описывают краевые возмущения ДКЭХ-жидкости на цилиндре. В разделе 7 объясняется, как мысленные эксперименты Лавлина по переносу заряда в холловском образце могут быть поняты с точки зрения КТП Люттингера. Это позволяет отождествить параметр взаимодействия КТП Люттингера с заполняющей фракцией жидкости Холла. В разделе 8 КТП Люттингера, отвечающие разложению в ряд Лавлина, связываются с РКТП. Этот анализ развивается в разделе 9, где вводится дробный заряд на краю, чтобы исследовать краевые возмушения, возникаюшие на фоне возмушений в слое жидкости Холла. Получаемая таким образом теория опять-таки есть РКТП и одновременно твистованная КТП Люттингера. Физический смысл алгебры максимальных симметрий этих РКТП обсуждается с точки зрения подхода Стоуна и Фишера к проблеме краевых фермионов в ДКЭХ.

Наконец, большинство технических деталей и вычислений приведено в приложениях. Приложение F содержит все необходимые определения и свойства эллиптических и модулярных функций, которые нам понадобятся в этой статье. В приложении G обсуждается простой случай нерелятивистских свободных фермионов и показывается согласованность результатов с результатами КТП. В приложении G приводятся также операторные вычисления, подобные рассуждениям Халдейна во взаимодействующей теории.

\section{2. КОНФОРМНАЯ ТЕОРИЯ ПОЛЯ ЖЛ}

В этом разделе мы напоминаем, как физика ЖЛ при больших расстояниях может быть описана эффективной конформной теорией поля, а именно теорией свободного бозонного поля, компактифицированного на окружность.

\section{1. Модель Люттингера}

ЖЛ - это теория одномерных взаимодействующих фермионов на окружности периметра $L$. Подробное описание низкоэнергетической физики ЖЛ было дано Халдейном [8].

В случае нерелятивистских фермионов квадратичное дисперсионное соотношение $\epsilon(k)=k^{2} / 2 m$ одномерного ферми-газа вблизи поверхности Ферми линеаризуется: $\epsilon_{\operatorname{lin}}(k)=v_{\mathrm{F}}\left(\alpha k-k_{\mathrm{F}}\right)$, где $\alpha=+1,-1$ (они соответственно отвечают $R, L$ - правым и левым точкам Ферми), а $k_{\mathrm{F}}$ является волновым вектором Ферми. Скорость Ферми задается соотношением

$$
v_{\mathrm{F}}=\frac{d \epsilon}{d k}\left(k_{\mathrm{F}}\right)
$$


Это приближение справедливо, если только взаимодействия не слишком сильны по сравнению со скоростью Ферми. Мы вернемся к этому вопросу в пункте 3.3.2. Затем линейные дисперсионные соотношения продолжаются на произвольно большие энергии, при этом наинизшее состояние содержит бесконечно большое число фермионов (море Дирака). (Имеются две линейные ветви.) Полученная система должна полностью описывать физику макроскопических расстояний исходной модели. Отметим, что результаты, полученные с помошью метода анзаца Бете, согласуются с указанной эффективной теорией [31].

2.1.1. Свободная теория. В инфракрасном пределе море Ферми заменяется морем Дирака, поэтому мы будем считать, что $k_{\mathrm{F}}=0$ (если бы $k_{\mathrm{F}} \neq 0$, то это лишь немного изменило бы ситуацию). Свободный гамильтониан имеет вид

$$
H^{(0)}=\frac{2 \pi v_{\mathrm{F}}}{L} \sum_{n, \alpha} \alpha n: c_{n, \alpha}^{\dagger} c_{n, \alpha}:
$$

где операторы $c$ - фермионные операторы рождения и уничтожения для импульса $2 \pi \alpha n / L$. Эти операторы подчиняются каноническим антикоммутационным соотношениям

$$
\begin{aligned}
& \left\{c_{n, \alpha}, c_{m, \alpha^{\prime}}\right\}=\left\{c_{n, \alpha}^{\dagger}, c_{m, \alpha^{\prime}}^{\dagger}\right\}=0, \\
& \left\{c_{n, \alpha}^{\dagger}, c_{m, \alpha^{\prime}}\right\}=\delta_{\alpha, \alpha^{\prime}} \delta_{n, m} \mathbf{1} .
\end{aligned}
$$

Фермионное нормальное упорядочение имеет вид $: c_{k}^{\dagger} c_{k}:=c_{k}^{\dagger} c_{k}$, если $k>0$, и $: c_{k}^{\dagger} c_{k}:=$ $-c_{k}^{\dagger} c_{k}$, если $k<0$. Мы задаем фермионные поля следуюшими формулами:

$$
\begin{aligned}
& \psi_{R}^{\dagger}(\sigma)=\frac{1}{\sqrt{L}} \sum_{n} c_{n, R}^{\dagger} e^{-2 \pi i n \sigma / L}, \\
& \psi_{L}^{\dagger}(\sigma)=\frac{1}{\sqrt{L}} \sum_{n} c_{n, L}^{\dagger} e^{2 \pi i n \sigma / L} .
\end{aligned}
$$

Как отмечено в приложении А, эти поля суть слабо изменяюшиеся в пространстве компоненты исходного поля Ферми, быстро же осциллируюшая часть появляется из осцилляций в волновом секторе Ферми колебаний. Операторы плотности заряда и тока имеют вид

$$
\begin{aligned}
& \rho(\sigma)=: \psi_{R}^{\dagger}(\sigma) \psi_{R}(\sigma)+\psi_{L}^{\dagger}(\sigma) \psi_{L}(\sigma): \\
& j(\sigma)=v_{\mathrm{F}}: \psi_{R}^{\dagger}(\sigma) \psi_{R}(\sigma)-\psi_{L}^{\dagger}(\sigma) \psi_{L}(\sigma):
\end{aligned}
$$

Энергия взаимодействия между электронами и внешним электростатическим или векторным потенциалом линейна:

$$
H_{\mathrm{ext}}=\int_{0}^{L} d \sigma(V(\sigma) \rho(\sigma)-A(\sigma) j(\sigma)) .
$$


Алгебра токов является главным методом в операторной формулировке модели. Определим следующие операторы $(n \in \mathbb{Z})$ :

$$
\begin{aligned}
& \mathcal{J}_{n}=\int_{0}^{L} \mathcal{J}(\sigma) e^{-2 \pi i n \sigma / L} d \sigma, \\
& \overline{\mathcal{J}}_{n}=\int_{0}^{L} \overline{\mathcal{J}}(\sigma) e^{2 \pi i n \sigma / L} d \sigma,
\end{aligned}
$$

где $\mathcal{J}(\sigma)=: \psi_{R}^{\dagger}(\sigma) \psi_{R}(\sigma):$ и $\overline{\mathcal{J}}(\sigma)=: \psi_{L}^{\dagger}(\sigma) \psi_{L}(\sigma):$. Можно показать, что моды $(2.9)$ и (2.10) удовлетворяют коммутационным соотношениям

$$
\left[\mathcal{J}_{n}, \mathcal{J}_{m}\right]=n \delta_{n,-m} \mathbf{1}
$$

и этот аномальный коммутатор лежит в центре бозонной алгебры. Его можно получить или с помощью регуляризации раздвижкой точек в вещественном пространстве (см., например, [54]), или непосредственно в фурье-пространстве (см., например, [8]). В обоих случаях аномалия возникает из-за нормального упорядочения операторов по отношению к морю Дирака. Более того, операторы $\mathcal{J}_{n}$ коммутируют с операторами $\overline{\mathcal{J}}_{m}$, которые также удовлетворяют коммутационным соотношениям (2.11). Эти соотношения задают бесконечномерную алгебру Гейзенберга [55], которая также называется аффиинной $U(1)$-алгеброй и обозначается $\widehat{U(1)}$. Таким образом, симметрии модели суть две коммутируюшие копии алгебры Гейзенберга ${ }^{1}$. Вакуумное состояние теории Дирака $|0\rangle$ удовлетворяет условиям старшего веса, которые формулируются как принцип исключения Паули:

$$
\forall n>0, \quad \mathcal{J}_{n}|0\rangle=\overline{\mathcal{J}}_{n}|0\rangle=0 .
$$

Наконец, как показал Халдейн в [8], свободный гамильтониан (2.1) может быть представлен через токи:

$$
H^{(0)}=\frac{\pi v_{\mathrm{F}}}{L} \sum_{n \in \mathbb{Z}}\left(\mathcal{J}_{n} \mathcal{J}_{-n}+\overline{\mathcal{J}}_{n} \overline{\mathcal{J}}_{-n}\right)=\pi v_{\mathrm{F}} \int_{0}^{L}\left(\mathcal{J}^{2}(\sigma)+\overline{\mathcal{J}}^{2}(\sigma)\right) d \sigma
$$

Эта бозонизованная форма свободного гамильтониана и есть главное выражение в технике бозонизации по модам.

2.1.2. Взаимодействующая теория. В дальнейшем мы полагаем, что взаимодействия между фермионами локальны, хотя у Халдейна можно найти решение и для нелокального взаимодействия при наложении некоторых специальных условий. Короткодействуюшие взаимодействия удовлетворяют этим условиям, а локальный случай может рассматриваться как предельный случай указанного анализа. Член с локальным взаимодействием в бесспиновой ЖЛ имеет вид

$$
H_{\mathrm{int}}=\frac{\pi}{L} \sum_{\alpha, \alpha^{\prime}} \sum_{k, k^{\prime}}\left(g_{4} \delta_{\alpha, \alpha^{\prime}}+g_{2} \delta_{\alpha,-\alpha^{\prime}}\right) c_{k+q, \alpha}^{\dagger} c_{k, \alpha} c_{k^{\prime}-q, \alpha^{\prime}}^{\dagger} c_{k^{\prime}, \alpha^{\prime}}
$$

\footnotetext{
1) Подобное расщепление алгебр левых и правых симметрий в КТП встречается часто.
} 
Полный гамильтониан диагонализуется с помощью процедуры бозонизации [8], используюшей понятие токовых мод, введенное в (2.9) и (2.10). Взаимодействия сначала переписьваются через токи:

$$
\begin{aligned}
H_{\text {int }} & =\frac{\pi}{L} \sum_{n \in \mathbb{Z}}\left(2 g_{2} \mathcal{J}_{n} \overline{\mathcal{J}}_{n}+g_{4}\left(\mathcal{J}_{n} \mathcal{J}_{-n}+\overline{\mathcal{J}}_{n} \overline{\mathcal{J}}_{-n}\right)\right)= \\
& =2 \pi g_{2} \int_{0}^{L} \mathcal{J}(\sigma) \overline{\mathcal{J}}(\sigma) d \sigma+\pi g_{4} \int_{0}^{L}\left(\mathcal{J}^{2}(\sigma)+\overline{\mathcal{J}}^{2}(\sigma)\right) d \sigma
\end{aligned}
$$

Теперь преобразование Боголюбова приводит к переопределению генераторов алгебры токов и диагонализует гамильтониан при подходяшем выборе угла. Более точно, определим

$$
\begin{aligned}
& J_{n}=\operatorname{ch}(\varphi) \mathcal{J}_{n}-\operatorname{sh}(\varphi) \overline{\mathcal{J}}_{-n}, \\
& \bar{J}_{n}=\operatorname{ch}(\varphi) \overline{\mathcal{J}}_{n}-\operatorname{sh}(\varphi) \mathcal{J}_{-n},
\end{aligned}
$$

где

$$
\operatorname{th}(2 \varphi)=-\frac{g_{2}}{v_{\mathrm{F}}+g_{4}} .
$$

Во взаимодействующей теории эти генераторы являются генераторами новой симметрии. Они также удовлетворяют коммутационным соотношениям (2.11). Генераторы $\mathcal{J}$ и $\overline{\mathcal{J}}$ понимаются как генераторы “голых" симметрий, а операторы $J$ и $\bar{J}$ - перенормированные операторы, которые отвечают за бесконечномерную аффинную симметрию в теории со взаимодействием. Сделаем фурье-преобразование лорановских мод $\left(J_{n}\right)_{n}$ и $\left(\bar{J}_{n}\right)_{n}$, которые при этом перейдут в $J(\sigma)$ и $\bar{J}(\sigma)$. При этом мы получим

$$
\left(\begin{array}{c}
J(\sigma) \\
\bar{J}(\sigma)
\end{array}\right)=\left(\begin{array}{cc}
\operatorname{ch}(\varphi) & -\operatorname{sh}(\varphi) \\
-\operatorname{sh}(\varphi) & \operatorname{ch}(\varphi)
\end{array}\right) \times\left(\begin{array}{c}
\mathcal{J}(\sigma) \\
\overline{\mathcal{J}}(\sigma)
\end{array}\right)
$$

В этих обозначениях свободньй гамильтониан вместе с членом взаимодействия будет просто гамильтонианом свободной бозонной теории поля:

$$
H_{\mathrm{tot}}=\frac{\pi v_{S}}{L} \sum_{n \in \mathbb{Z}}\left(J_{n} J_{-n}+\bar{J}_{n} \bar{J}_{-n}\right)=\pi v_{S} \int_{0}^{L}\left(J^{2}(\sigma)+\bar{J}^{2}(\sigma)\right) d \sigma .
$$

Оператор плотности не модифицируется при включении взаимодействия, поскольку он является просто оператором числа частиш:

$$
\rho(\sigma)=: \psi_{R}^{\dagger}(\sigma) \psi_{R}(\sigma):+: \psi_{L}^{\dagger}(\sigma) \psi_{L}(\sigma):=\alpha^{-1 / 2}(J(\sigma)+\bar{J}(\sigma)) .
$$

В этой формуле мы ввели параметр взаимодействия $\alpha=e^{-2 \varphi}$. Для построения тока полезно, как показал Халдейн в работе [8], применять закон сохранения тока. Используя 
полный взаимодействующий гамильтониан и коммутационные соотношения для операторов $J(\sigma)$ и $\bar{J}(\sigma)$, мы можем легко найти эволюцию по времени этих операторов в представлении Гейзенберга ${ }^{2)}$ :

$$
\begin{aligned}
& J(\sigma, t)=\frac{1}{L} \sum_{n \in \mathbb{Z}} J_{n} e^{2 \pi i n\left(\sigma-v_{S} t\right) / L}, \\
& \bar{J}(\sigma, t)=\frac{1}{L} \sum_{n \in \mathbb{Z}} \bar{J}_{n} e^{-2 \pi i n\left(\sigma+v_{S} t\right) / L} .
\end{aligned}
$$

Мы учитываем взаимодействия посредством модификации скорости Ферми $v_{\mathrm{F}} \mapsto v_{S}$, причем

$$
\begin{aligned}
v_{S} & =\sqrt{\left(v_{\mathrm{F}}+g_{4}\right)^{2}-g_{2}^{2}}, \\
v_{N} & =v_{\mathrm{F}}+g_{2}+g_{4}, \\
v_{J} & =v_{\mathrm{F}}-g_{2}+g_{4} .
\end{aligned}
$$

Тогда $\alpha=\sqrt{v_{N} / v_{J}}$. Подводя итог, мы получили

$$
\begin{aligned}
& \rho(\sigma)=\alpha^{-1 / 2}(J(\sigma)+\bar{J}(\sigma)), \\
& j(\sigma)=v_{S} \alpha^{-1 / 2}(J(\sigma)-\bar{J}(\sigma)) .
\end{aligned}
$$

Разумеется, при этих вычислениях предполагается, что взаимодействия локальны, т.е. что $\varphi$ не зависит от $n$. Поскольку мы интересуемся низкоэнергетическими свойствами модели, то будем предполагать, что эта гипотеза верна, по крайней мере для мод, дающих вклад в возбуждения, существенные в исследуемом диапазоне температур.

Вакуум теории со взаимодействием $\left|O_{\text {Lutt }}\right\rangle$ также удовлетворяет условиям старшего веса $(2.12)$, но уже для операторов $J_{n}$ и $\bar{J}_{n}$ (подробная информация о теории представлений бесконечномерных алгебр Ли содержится в книге [55]). Как хорошо известно, это состояние ортогонально исходному вакуумному состоянию. При нелокальных взаимодействиях угол $\varphi$ зависит от $n$. Для сохранения регуляризации мы выпишем зависимость $\varphi$ от $n^{3)}$ :

$$
\left|O_{\text {Lutt }}\right\rangle=\mathcal{Z}^{-1 / 2} \exp \left(-\sum_{n=1}^{+\infty} \frac{\operatorname{th}\left(\varphi_{n}\right)}{n} \mathcal{J}_{-n} \overline{\mathcal{J}}_{-n}\right)|0\rangle
$$

\footnotetext{
2) Распространение волнового пакета, созданного при нулевом времени оператором

$$
\psi_{R}^{\dagger}[\varphi(\sigma)](t=0)=\int \varphi(\sigma) \psi_{R}^{\dagger}(\sigma, 0) d \sigma
$$

имеет вид

$$
e^{-i t H / \hbar} \psi_{R}^{\dagger}[\varphi(\sigma)] e^{i t H / \hbar}=\psi_{R}^{\dagger}\left[f\left(\sigma-v_{S} t\right)\right] .
$$

Как и следовало ожидать, волновые пакеты правых частиц распространяются в правом направлении.

${ }^{3)}$ Следует помнить, что при больших $n$ в теории всегда имеется некоторое ультрафиолетовое обрезание.
} 
Нормирующий множитель $\mathcal{Z}$ имеет вид

$$
\mathcal{Z}=\prod_{n=1}^{+\infty} \operatorname{ch}^{2}\left(\varphi_{n}\right)
$$

Множитель $\mathcal{Z}$ расходится в термодинамическом пределе, когда все больше и больше мод рождается ниже порога ультрафиолетового обрезания. Следовательно, вакуум теории со взаимодействием ортогонален вакууму свободной теории. Заметим, однако, что пространство Гильберта все еше является представлением алгебры $\widehat{U(1)} \times \widehat{U(1)}_{L}$, порожденной “перенормированными” токами $J_{n}$ и $\bar{J}_{n}$. При этом включение взаимодействия сохраняет симметрию эффективной теории Люттингера.

Теперь диагонализованньй гамильтониан имеет вид

$$
H_{\text {Lutt }}=E_{0}+\frac{2 \pi v_{S}}{L} \sum_{n>0} n\left(N_{n, R}+N_{n, L}\right)+\frac{\pi v_{S}}{2 L}\left(\alpha N^{2}+\frac{1}{\alpha} J^{2}\right),
$$

где $E_{0}$ обозначает вакуумную энергию. В формуле (2.30) числа $N_{n, R}$ и $N_{n, L}$ - числа заполнения для бозонных мод:

$$
\forall n>0, \quad N_{R, n}=\frac{1}{n} J_{-n} J_{n}, \quad N_{n, L}=\frac{1}{n} \bar{J}_{-n} \bar{J}_{n} .
$$

Операторы $N$ и $J$ - зарядовые и токовые числа моды $q=0: N=J_{0}+\bar{J}_{0}$ и $J=J_{0}-\bar{J}_{0}$.

Заметим, что физика ЖЛ задается двумя параметрами: перенормированной скоростью Ферми $v_{S}$ и безразмерным параметром взаимодействия $\alpha$. В точке, отвечаюшей жидкости Ферми, эти параметры принимают значения $v_{S}=v_{\mathrm{F}}$ и $\alpha=1$.

\section{2. Теоретико-полевое описание}

Здесь мы опишем теоретико-полевой подход к ЖЛ. Как было показано выше, все физические величины этой квантовой системы при конечной температуре могут быть выведены из двумерной евклидовой квантовой теории поля, которая будет дана в следуюших разделах.

2.2.1. КТП в размерности два. Основная идея - это представить одномерную квантовую систему при конечной температуре через двумерную статистическую теорию поля. Эта достаточно старая идея [21] оказалась полезной для описания ЖЛ, поскольку последняя обладает бесшелевыми возмушениями. Таким образом, можно надеяться, что соответствующая двумерная статистическая теория будет масштабно-инвариантной и, более того, конформно-инвариантной ${ }^{4)}$. Перед тем как исследовать эффективную КТП, которая описывает низкоэнергетическую физику ЖЛ, приведем некоторые основные свойства КТП [20, 23, 24, 26].

Как обычно в КТП, мы будем интересоваться статистическими суммами со вставками специального оператора твиста: кроме оператора $\exp (-\beta H)$, мы вводим оператор

\footnotetext{
4) Важно не забывать, что эти доводы вполне эвристичны. Их значимость основана на том, что мы можем получить халдейновский спектр и другие известные низкоэнергетические свойства ЖЛ с использованием КТП.
} 
сдвига в пространственном направлении $\exp (i \theta P)$. Более точно, мы будем вычислять статистические суммы вида $Z=\operatorname{Tr}\left(e^{-\beta H} e^{i \theta P}\right)$. Как показал Карди в [22], это отвечает вычислению функциональных интегралов на торе. В нашем случае соответствуюший модулярный параметр $\tau$ этого тора имеет вид

$$
\tau=\frac{\theta}{L}+i \frac{\beta v_{S}}{L}
$$

Как обычно, в КТП [22] операторы гамильтониана и импульса могут быть представлены через генераторы алгебры Вирасоро $L_{0}$ и $\bar{L}_{0}$ следующим образом:

$$
\begin{aligned}
& H=\frac{2 \pi v_{S}}{L}\left(L_{0}+\bar{L}_{0}-\frac{c}{12}\right) \\
& P=\frac{2 \pi v_{S}}{L}\left(L_{0}-\bar{L}_{0}\right) .
\end{aligned}
$$

Число $c$ (центральный заряд) отвечает энергии низшего состояния. Ответы, как правило, выражаются через комплексные параметры $q=\exp (2 i \pi \tau)$ и $\bar{q}=\exp (-2 i \pi \bar{\tau})$. Например, статистическая сумма

$$
Z=\operatorname{Tr}\left(q^{L_{0}-\frac{1}{24}} \bar{q}^{\bar{L}_{0}-\frac{1}{24}}\right)
$$

может быть представлена через двойное разложение Пуисо по параметрам $q$ и $\bar{q}$. Коэффициенты этого разложения являются числами вырождения состояний с заданной энергией и импульсом.

2.2.2. Свободные фермионы на торе. Гамильтониан (2.1) возникает в теории Дирака свободных фермионов. Введем гамма-матрищы пространства Минковского: $\gamma_{0}=$ $\sigma_{x}$ и $\gamma_{1}=-i \sigma_{y}$. Лагранжиан дираковских фермионов имеет вид

$$
\mathcal{L}=i \bar{\psi} \gamma^{\mu} \partial_{\mu} \psi
$$

где $\bar{\psi}=\psi^{\dagger} \gamma^{0}$. Мы будем интересоваться термодинамикой ЖЛ. Помимо операторного подхода, в котором главную роль играет оператор плотности $\exp (-\beta H)$, будет также активно использоваться функциональный подход и выгисляться функциональные интегралы Березина вида

$$
\int \mathcal{D}[\bar{\psi}, \psi] \exp \left(-\int \bar{\psi} \gamma_{\mathrm{E}}^{\mu} \partial_{\mu} \psi\right)
$$

где через $\left(\gamma_{\mathrm{E}}^{\mu}\right)_{\mu}$ обозначены евклидовы гамма-матрицы $\left(\gamma_{\mathrm{E}}^{0}=\sigma_{x}\right.$ и $\left.\gamma_{\mathrm{E}}^{1}=\sigma_{y}\right)$.

В фермионном интеграле, задаюшем статистическую сумму данной системы, евклидово действие есть интеграл по тору $S_{1} \times S_{1}$. Пространственное направление ассоциируется с первым кругом, а вторая образуюшая отвечает направлению мнимого времени. Очевидно, что граничные условия для фермионных полей на торе играют важную роль, которую мы и обсудим ниже. 
Пространственные граничные условия. В пространственном направление фермионы могут быть периодичны $(n \in \mathbb{Z})$ или антипериодичны $(n \in \mathbb{Z}+1 / 2)$ [56]. В последнем случае возникает четырехкратное вырождение уровней, поскольку если фермионные уровни расположены на поверхности Ферми, то полная энергия не зависит от заполненности этих уровней. В работе [8] волновой вектор Ферми был полуцелым, поэтому впредь мы будем рассматривать фермионы в антипериодическом секторе.

Интересно тем не менее рассмотреть оба случая. Определим в каждом из этих двух "периодических" и "антипериодических" случаев свои гильбертовы пространства, обозначаемые соответственно как $\mathcal{H}_{\mathrm{P}}$ и $\mathcal{H}_{\mathrm{A}}$. Как мы увидим в дальнейшем, при пропускании магнитного потока через кольцо можно получать и другие граничные условия.

Граничные условия при мнимом времени. В формализме функционального интеграла (см. [57, гл. 9]) вычисление следа оператора, действуюшего в отдельной фермионной моде, сводится к вычислению интеграла Березина от ядра этого оператора с антипериодическими граничными условиями. Напомним, что периодические граничные условия получатся, если ввести множитель $(-1)^{F}$, где $F$ - фермионное число:

$$
\begin{aligned}
& \operatorname{Tr}(A)=\int d \bar{\xi} d \xi e^{\xi \bar{\xi}} A(-\bar{\xi}, \xi), \\
& \operatorname{Tr}\left((-1)^{F} A\right)=\int d \bar{\xi} d \xi e^{\xi \bar{\xi}} A(\bar{\xi}, \xi) .
\end{aligned}
$$

Таким образом, в нашем подходе получатся четыре различных типа граничных условий, накладываемых на фермионы на торе: PР, PA, АР и АА, где первый индекс обозначает тип периодичности по пространственной координате, а второй - по направлению мнимого времени. Мы будем называть эти условия “спиновыми секторами”. Окончательно, получим

$$
\begin{aligned}
& Z_{\mathrm{AP}}=\operatorname{Tr}_{A}\left((-1)^{F} e^{-\beta H_{A}}\right)=\int_{\mathrm{AP}} \mathcal{D}[\bar{\psi}, \psi] \exp \left(-\int \bar{\psi} \gamma_{\mathrm{E}}^{\mu} \partial_{\mu} \psi\right), \\
& Z_{\mathrm{PP}}=\operatorname{Tr}_{P}\left((-1)^{F} e^{-\beta H_{P}}\right)=\int_{\mathrm{PP}} \mathcal{D}[\bar{\psi}, \psi] \exp \left(-\int \bar{\psi} \gamma_{\mathrm{E}}^{\mu} \partial_{\mu} \psi\right), \\
& Z_{\mathrm{PA}}=\operatorname{Tr}_{P}\left(e^{-\beta H_{P}}\right)=\int_{\mathrm{PA}} \mathcal{D}[\bar{\psi}, \psi] \exp \left(-\int \bar{\psi} \gamma_{\mathrm{E}}^{\mu} \partial_{\mu} \psi\right), \\
& Z_{\mathrm{AA}}=\operatorname{Tr}_{A}\left(e^{-\beta H_{A}}\right)=\int_{\mathrm{AA}} \mathcal{D}[\bar{\psi}, \psi] \exp \left(-\int \bar{\psi} \gamma_{\mathrm{E}}^{\mu} \partial_{\mu} \psi\right) .
\end{aligned}
$$

Мы исследуем двумерную безмассовую теорию Дирака, являющуюся КТП. Однако в отличие от обычного струнного подхода, мы ограничиваем рассмотрение одним выделенным спиновым сектором и не берем сумму по всем возможностям.

2.2.3. Безмассовая модель Тирринга. Инфракрасный предел описывается теорией со взаимодействием, выраженной суммой гамильтонианов (2.1) и (2.15). В теории Дирака сохраняющийся ток задается формулой $J^{\mu}=\bar{\psi} \gamma^{\mu} \psi$. Член взаимодействия в гамильтониане Халдейна выражается через этот ток и соответствует, таким образом, 
квадратичному взаимодействию токов, имеющему в лагранжевой формулировке форму Тирринга:

$$
S_{\mathrm{int}}[\bar{\psi}, \psi]=-\frac{\kappa}{2} \int\left(\bar{\psi} \gamma^{\mu} \psi\right)^{2}
$$

Таким образом, евклидова квантовая теория поля, рассматриваемая в данной работе, описывает безмассовую модель Тирринга [58] в специальном спиновом секторе, который мы называем КТП Люттингера. Ее лагранжиан имеет вид

$$
\mathcal{L}=i \bar{\psi} \gamma^{\mu} \partial_{\mu} \psi-\frac{\kappa}{2}\left(\bar{\psi} \gamma^{\mu} \psi\right)^{2}
$$

Анализ, приведенный в разделах 5 и 6 , состоит в выполнении явных вычислений корреляционных функции в рамках функциональной процедуры бозонизации. Определяющими параметрами теории являются скорость Ферми и константа связи $\kappa$. Они соотносятся c $v_{S}$ и $\alpha$ в подходе Халдейна. Чтобы установить полное соответствие между подходом Халдейна, вычислениями в подходе бозонизации и моделью Тирринга, нужно уметь явно вычислять корреляционные функции в безмассовой модели Тирринга ${ }^{5}$. (По этому вопросу см. работы [59] и [60]). Эти выгисления основаны на правильном определении составных токовых операторов $\bar{\psi} \gamma^{\mu} \psi$ и $\bar{\psi} \gamma^{\mu} \gamma^{5} \psi$. Однако, поскольку нам известны спектр и асимптотики фермионных корреляторов как в подходе Халдейна, так и в бозонном подходе, все что нам осталось, - это использовать соотношения (2.23)-(2.25) и определение $\alpha$. В будушем было бы интересно прояснить соотношения между этими тремя подходами.

2.2.4. Явные вычисления в операторном формализме. Представляет интерес явное вычисление статистических сумм (2.39) и (2.40) в теории Дирака с использованием операторного формализма. В этом пункте вычисления проводятся при нулевых магнитном поле и химическом потенциале. Более подробное обсуждение данного вопроса содержится в приложении В.

Определение операторов. Чтобы привести описание обеих ветвей решения к единому виду, мы вводим обозначения $b_{n, R}^{\dagger}=c_{n, R}^{\dagger}, b_{n, L}^{\dagger}=c_{-n, L}^{\dagger}$ и $N_{n, \alpha}=b_{n, \alpha}^{\dagger} b_{n, \alpha}$, если $n>0$, $N_{n, \alpha}=b_{n, \alpha} b_{n, \alpha}^{\dagger}$, если $n<0$. Затем, используя дзета-функциональную перенормировку для устранения расходимостей, мы получим

$$
H=\frac{2 \pi v_{\mathrm{F}}}{L} \sum_{n}|n|\left(N_{n, R}+N_{n, L}\right)-2 \zeta_{a}(-1)
$$

где $a=0$ отвечает периодическому сектору и $a=-1 / 2-$ антипериодическому. В этом выражении $\zeta_{a}-$ аналитическое продолжение функции:

$$
\zeta_{a}(s)=\sum_{n=1}^{+\infty} \frac{1}{(n+a)^{s}}
$$

5) Это замечание сделал Стора. 
Правая часть уравнения (2.46) определена при достаточно больших $\Re(s)$. Напомним, что [61]

$$
\zeta_{0}(-1)=-\frac{1}{12} \quad \text { и } \quad \zeta_{1 / 2}(-1)=\frac{1}{24} .
$$

Теперь гамильтониан полностью определен в каждом секторе:

$$
\begin{aligned}
& H_{P}=\frac{2 \pi v_{\mathrm{F}}}{L} \sum_{n \in \mathbb{Z}}|n|\left(N_{n, R}+N_{n, L}\right)+\frac{1}{6}, \\
& H_{A}=\frac{2 \pi v_{\mathrm{F}}}{L} \sum_{n \in \mathbb{Z}+1 / 2}|n|\left(N_{n, R}+N_{n, L}\right)-\frac{1}{12} .
\end{aligned}
$$

Оператор импульса не зависит от регуляризашии и имеет вид

$$
P=\frac{2 \pi v_{\mathrm{F}}}{L} \sum_{n}|n|\left(N_{n, R}-N_{n, L}\right) .
$$

Явные выражения для статистических сумм. В свободном случае все следы явно вычисляются:

$$
\begin{aligned}
& Z_{\mathrm{AA}}=(q \bar{q})^{-1 / 24}\left|\prod_{n=0}^{+\infty}\left(1+q^{n+1 / 2}\right)^{2}\right|^{2}, \\
& Z_{\mathrm{PA}}=4(q \bar{q})^{-1 / 24}\left|q^{1 / 8} \prod_{n=1}^{+\infty}\left(1+q^{n}\right)^{2}\right|^{2}, \\
& Z_{\mathrm{AP}}=(q \bar{q})^{-1 / 24}\left|\prod_{n=0}^{+\infty}\left(1-q^{n+1 / 2}\right)^{2}\right|^{2}, \\
& Z_{\mathrm{PP}}=0 .
\end{aligned}
$$

Статистическая сумма $Z_{\mathrm{PP}}$ равна нулю, поскольку в дважды периодическом секторе число заполнения состояния, лежашего на поверхности Ферми, не влияет ни на энергию, ни на импульс (т.к. $\left.k_{\mathrm{F}}=0\right)$. На языке функционального интегрирования это отвечает сушествованию в спиновом секторе РР нулевых фермионных мод.

Заметим, что эти статистические суммы могли бы также быть получены в каждом спиновом секторе с помошью дзета-регуляризации детерминанта оператора Дирака на Tope [24].

Статистические суммы ЖЛ. Получим теперь из спектра Халдейна статистическую сумму взаимодействуюшей теории Люттингера. С помошью $\zeta$-перенормировочного описания мы получим

$$
E_{0}=-\frac{\pi v_{S}}{6 L}
$$

После введения функции Дедекинда

$$
\eta(q)=q^{\frac{1}{24}} \prod_{n=1}^{+\infty}\left(1-q^{n}\right)
$$


статистическая сумма ЖЛ принимает вид

$$
Z_{\mathrm{AA}}=\frac{1}{|\eta(q)|^{2}}\left(\sum_{\substack{(n, m) \in \mathbb{Z}^{2} \\ m \equiv 0(2)}}+\sum_{\substack{(n, m) \in(\mathbb{Z}+1 / 2) \times \mathbb{Z} \\ m \equiv 1(2)}}\right) q^{\frac{1}{2}\left(n \sqrt{\alpha}+\frac{m}{2 \sqrt{\alpha}}\right)^{2}} \bar{q}^{\frac{1}{2}\left(n \sqrt{\alpha}-\frac{m}{2 \sqrt{\alpha}}\right)^{2}}
$$

где мы просто заменили сумму по $J$ и $N$, отвечающим одной и той же четности, суммой по $(n, m)$ с $N=2 n+m$ и $J=2 n-m$. Проблема теперь - найти удобный способ описания физики ЖЛ. Более точно, мы хотим найти эффективную теорию поля, которая могла бы воспроизвести статистическую сумму (2.57) с помощью функционального интегрирования.

\section{3. Бозонное описание эффективной теории Люттингера}

Опишем теперь, как найти статистическую сумму взаимодействующей теории Люттингера с помощью свободной бозонной теории. Все трудности обусловлены граничными условиями бозонного поля, к которым мы сейчас и обратимся. Для начала напомним, как вычислять статистические суммы в компактифицированной бозонной теории, а затем получим фермионную статистическую сумму АА. С помошью стандартных методов модулярной ковариантности мы получим затем явные выражения в двух оставшихся секторах.

2.3.1. Компактифицированный бозон. Здесь мы приводим явные выражения для статистических сумм в теории свободного бозона, компактифицированного на окружность радиуса $R$ [27], т.е. мы идентифицируем $\varphi$ и $\varphi+2 \pi R$. Поле задано на тоpe $\mathbf{T}_{\Gamma}=\mathbb{C} / \Gamma$, где $\Gamma$ обозначает решетку $\Gamma=\omega_{1} \mathbb{Z}+\omega_{2} \mathbb{Z}$. Мнимая часть модулярного параметра $\tau=\omega_{2} / \omega_{1}$ положительна. Действие имеет вид

$$
S[\varphi]=\frac{g}{2 \pi} \int_{\mathbf{T}_{\Gamma}}|\nabla \varphi|^{2}
$$

Возможные граничные условия $\left[\epsilon, \epsilon^{\prime}\right]$, где $\epsilon$ и $\epsilon^{\prime}$ заданы по модулю 1 , задаются условиями монодромии:

$$
\begin{aligned}
& \varphi\left(z+\omega_{1}\right) \equiv \varphi(z)+2 \pi R \epsilon(\bmod 2 \pi R), \\
& \varphi\left(z+\omega_{2}\right) \equiv \varphi(z)+2 \pi R \epsilon^{\prime}(\bmod 2 \pi R) .
\end{aligned}
$$

Очевидно, что статистическая сумма

$$
Z_{\epsilon, \epsilon^{\prime}}=\int_{\left[\epsilon, \epsilon^{\prime}\right]} \mathcal{D}[\varphi] e^{-S[\varphi]}
$$

задается гауссовым интегралом, который может быть явно вычислен, если мы воспользуемся квадратичным порядком разложения вблизи классических решений (также называемых инстантонами), которые совместны с наложенными граничными условиями. Это вычисление хорошо известно и может быть явно проделано. Его детали содержатся в приложениях $\mathrm{C}$ и D. 
Выражение для бозонных статистических сумм. Как показано в приложении D, статистическая сумма для компактифицированного бозона имеет вид

$$
Z_{\left[\epsilon, \epsilon^{\prime}\right]}\left(g R^{2}\right)=\frac{1}{|\eta(q)|^{2}} \sum_{\substack{m \in \mathbb{Z} \\ n \in \mathbb{Z}}} e^{-2 i \pi m \epsilon^{\prime}} q^{\frac{1}{2} p_{n+\epsilon, m}^{2}} \bar{q}^{\frac{1}{2} \bar{p}_{n+\epsilon, m}^{2}},
$$

где введены импульсы

$$
\begin{aligned}
& p_{n, m}=n \sqrt{\alpha}+\frac{m}{2 \sqrt{\alpha}}, \\
& \bar{p}_{n, m}=n \sqrt{\alpha}-\frac{m}{2 \sqrt{\alpha}} .
\end{aligned}
$$

Выражение (2.61) - отправная точка всех вычислений в данной статье. Как мы увидим в дальнейшем, оно будет использоваться при вычислении корреляционных функции так называемых вершинных операторов, в том числе перенормированных фермионов.

2.3.2. Отождествление ЖЛ с компактифицированными бозонами. В теории струн основным объектом является модулярно-инвариантная статистическая сумма [24]

$$
Z_{\mathrm{Dirac}}=\frac{1}{2}\left(Z_{\mathrm{AA}}+Z_{\mathrm{AP}}+Z_{\mathrm{PA}}+Z_{\mathrm{PP}}\right)
$$

Эта сумма явно вычисляется обычным образом [24], она равна

$$
Z_{\text {Dirac }}=\frac{1}{|\eta(q)|^{2}} \sum_{(m, n) \in \mathbb{Z}^{2}} q^{\frac{1}{8}(n+2 m)^{2}} \bar{q}^{\frac{1}{8}(n-2 m)^{2}} .
$$

Сравнение с (2.61) дает [27]

$$
Z_{\text {Dirac }}=Z_{[0,0]}(1) .
$$

Теория Дирака, таким образом, совпадает с теорией свободного компактифишированного бозона при $g R^{2}=1$. Теперь задача - выразить каждую статистическую сумму $(2.51),(2.52)$ и $(2.53)$ в терминах бозонных функциональных интегралов с $g R^{2}=1$. Эти формулы уже встречались в литературе, по крайней мере в [34] и [33]. Весьма подробное исследование бозонизации для общего заряда и фоновой метрики можно найти в [38]. K сожалению, эти результаты по-видимому не слишком широко известны, и потому представляет интерес вывести их с помошью элементарных методов.

Бозонизованные выражения свободных статистических сумм. Тождество тройного произведения Якоби имеет вид [62]

$$
\sum_{n=-\infty}^{+\infty} y^{n} q^{n^{2} / 2}=\prod_{n=1}^{+\infty}\left(1-q^{n}\right) \prod_{n=0}^{+\infty}\left(1+y q^{n+1 / 2}\right)\left(1+y^{-1} q^{n+1 / 2}\right)
$$


Подставляя $y=1, y=q^{1 / 2}$ и $y=-1$ в совокупности с $(2.51),(2.52)$ и (2.53), мы получим выражения, которые, как будет показано, могут быть выведены из бозонизованной теории:

$$
\begin{aligned}
& Z_{\mathrm{AA}}=\frac{1}{|\eta(q)|^{2}}\left|\sum_{n=-\infty}^{+\infty} q^{\frac{1}{2} n^{2}}\right|^{2}, \\
& Z_{\mathrm{PA}}=\frac{1}{|\eta(q)|^{2}}\left|\sum_{n=-\infty}^{+\infty} q^{\frac{1}{2}\left(n+\frac{1}{2}\right)^{2}}\right|^{2}, \\
& Z_{\mathrm{AP}}=\frac{1}{|\eta(q)|^{2}}\left|\sum_{n=-\infty}^{+\infty}(-1)^{n} q^{\frac{1}{2} n^{2}}\right|^{2} .
\end{aligned}
$$

Выражения в терминах функционального интеграла. Теперь бозонные статистические суммы будут отождествляться с бозонными функциональными интегралами вида (2.61). Для этой цели выпишем явно (2.61) в указанных четырех секторах:

$$
\begin{aligned}
& Z_{[0,0]}(1)=\frac{1}{|\eta(q)|^{2}} \sum_{(n, m) \in \mathbb{Z}^{2}} q^{\frac{1}{2}\left(n+\frac{m}{2}\right)^{2}} \bar{q}^{\frac{1}{2}\left(n-\frac{m}{2}\right)^{2}}, \\
& Z_{\left[0, \frac{1}{2}\right]}(1)=\frac{1}{|\eta(q)|^{2}} \sum_{(n, m) \in \mathbb{Z}^{2}}(-1)^{m} q^{\frac{1}{2}\left(n+\frac{m}{2}\right)^{2}} \bar{q}^{\frac{1}{2}\left(n-\frac{m}{2}\right)^{2}}, \\
& Z_{\left[\frac{1}{2}, 0\right]}(1)=\frac{1}{|\eta(q)|^{2}} \sum_{(n, m) \in \mathbb{Z}^{2}} q^{\frac{1}{2}\left(n+\frac{1}{2}+\frac{m}{2}\right)^{2}} \bar{q}^{\frac{1}{2}\left(n+\frac{1}{2}-\frac{m}{2}\right)^{2}}, \\
& Z_{\left[\frac{1}{2}, \frac{1}{2}\right]}(1)=\frac{1}{|\eta(q)|^{2}} \sum_{(n, m) \in \mathbb{Z}^{2}}(-1)^{m} q^{\frac{1}{2}\left(n+\frac{1}{2}+\frac{m}{2}\right)^{2}} \bar{q}^{\frac{1}{2}\left(n+\frac{1}{2}-\frac{m}{2}\right)^{2}} .
\end{aligned}
$$

После некоторых вычислений, статистическая сумма свободной теории Дирака типа АА примет вид

$$
Z_{\mathrm{AA}}=\frac{1}{2}\left(Z_{[0,0]}(1)+Z_{\left[0, \frac{1}{2}\right]}(1)+Z_{\left[\frac{1}{2}, 0\right]}(1)-Z_{\left[\frac{1}{2}, \frac{1}{2}\right]}(1)\right) .
$$

После модулярного преобразования $\tau \rightarrow \tau+1$ и $\tau \rightarrow-1 / \tau$ из уравнения (2.75) получится

$$
\begin{aligned}
& Z_{\mathrm{AP}}=\frac{1}{2}\left(Z_{[0,0]}(1)+Z_{\left[0, \frac{1}{2}\right]}(1)-Z_{\left[\frac{1}{2}, 0\right]}(1)+Z_{\left[\frac{1}{2}, \frac{1}{2}\right]}(1)\right), \\
& Z_{\mathrm{PA}}=\frac{1}{2}\left(Z_{[0,0]}(1)-Z_{\left[0, \frac{1}{2}\right]}(1)+Z_{\left[\frac{1}{2}, 0\right]}(1)+Z_{\left[\frac{1}{2}, \frac{1}{2}\right]}(1)\right) .
\end{aligned}
$$

Главное наблюдение состоит в том, что каждый сектор фермионной теории может быть получен из сектора АА с помощью модулярного преобразования. С другой стороны, модулярные свойства бозонной статистической суммы $Z_{\left[\epsilon, \epsilon^{\prime}\right]}$ очевидны. 
Случай взаимодействия. При рассмотрении этого случая мы используем форму (2.42) статистической суммы ЖЛ, чтобы получить

$$
Z_{A A}^{(\mathrm{Lutt})}=\frac{1}{2}\left(Z_{[0,0]}(\sqrt{\alpha})+Z_{\left[0, \frac{1}{2}\right]}(\sqrt{\alpha})+Z_{\left[\frac{1}{2}, \frac{1}{2}\right]}(\sqrt{\alpha})-Z_{\left[\frac{1}{2}, 0\right]}(\sqrt{\alpha})\right) .
$$

ЖЛ с зарядовой скоростью $v_{N}$ и скоростью тока $v_{J}$ можно отождествить с компактифицированным бозоном с $g R^{2}=\sqrt{\alpha}$, где $\alpha=\sqrt{v_{N} / v_{J}}$. При этом нужно учитывать специфические граничные условия, наложенные на бозонное поле.

Конформные спины, возникающие в (2.78), имеют вид

$$
\Delta_{n, m}=\frac{1}{2}\left(n \sqrt{\alpha}+\frac{m}{2 \sqrt{\alpha}}\right)^{2}-\frac{1}{2}\left(n \sqrt{\alpha}-\frac{m}{2 \sqrt{\alpha}}\right)^{2}=n m .
$$

Таким образом, в задаче со взаимодействием мы не рассматриваем "экзотических" статистик, все поля имеют целый или полуцелый конформный спин.

Уравнение (2.78) описывает отождествление спектра ЖЛ, исследованного Халдейном, и спектра определенной бозонной КТП. Это соответствие основано на явном сравнении статистических сумм. Взаимодействие системы Люттингера с электромагнитным полем вводится для описания воздействия магнитного поля и электрического потенциала. Фермионные вычисления приводятся в приложении В. Там показано, что сектор АА не приводит к каким-либо сюрпризам. Однако, как показано в приложении В, сектор РР имеет особенности. В следуюшем разделе этот анализ расширяется на исследование бозонной теории поля Люттингера, взаимодействуюшей с калибровочным полем.

\section{3. ВЗАИМОДЕЙСТВИЕ С КАЛИБРОВОЧНЫМ ПОЛЕМ}

В этом разделе описьвается бозонизационная процедура для ЖЛ, взаимодействующей с калибровочным полем. Сначала мы исследуем компактифицированный бозон, взаимодействуюший с калибровочным полем, а потом отождествляем заряд и ток со структурами бозонного поля. В качестве приложения вычисляется статистическая сумма ЖЛ в присутствии магнитного потока через кольцо Люттингера. Впоследствии в разделе 5 будут получены явные выражения для производяших функционалов корреляторов плотностей зарядов и токов.

\section{1. Компактифицированный бозон, взаимодействующий с постоянным калибровочным полем}

В бозонной теории калибровочное поле присоединяется с помошью внешнего произведения, а именно

$$
S_{\wedge}[\varphi, A]=\frac{g}{2 \pi} \int(\nabla \varphi)^{2}-\frac{i}{\pi R} \int A \wedge d \varphi .
$$

Сначала рассмотрим случай постоянного калибровочного потенциала с голономиями

$$
\int_{(a)} A=2 \pi a \quad \text { и } \quad \int_{(b)} A=2 \pi b .
$$


На втором этапе в калибровочный потенциал будут введены переменные члены.

Вычислим статистическую сумму с граничными условиями (2.59). Детали вычисления, приведенные в приложении $\mathrm{D}$, дают следующий результат:

$$
Z_{\left[\epsilon, \epsilon^{\prime}\right]}[A]=\frac{1}{|\eta(q)|^{2}} \sum_{(m, n) \in \mathbb{Z}^{2}} e^{2 i \pi m \epsilon^{\prime}} e^{4 i \pi(\epsilon+n) b} q^{\frac{1}{2} p_{n+\epsilon, m+2 a}^{2}} \bar{q}^{\frac{1}{2} \bar{p}_{n+\epsilon, m+2 a}^{2},}
$$

где $p_{n, m}$ и $\bar{p}_{n, m}$ определены формулами (2.62) и (2.63).

В качестве упражнения рассмотрим невзаимодействующий случай, для которого $\alpha=g R^{2}=1$, и вычислим статистическую сумму

$$
Z[A]=\frac{1}{2} \sum_{\left(\epsilon, \epsilon^{\prime}\right) \in\{0,1 / 2\}^{2}}(-1)^{4 \epsilon \epsilon^{\prime}} Z_{\epsilon, \epsilon^{\prime}}[A] .
$$

В этом выражении знаки соответствуют знакам в тождестве (2.78). Отметим, что

$$
\sum_{\epsilon^{\prime} \in\{0,1 / 2\}}(-1)^{4 \epsilon \epsilon^{\prime}} e^{e i \pi m \epsilon^{\prime}}=\frac{1}{2}\left(1+(-1)^{2 \epsilon+m}\right)
$$

Таким образом, $m \equiv 2 \epsilon(\bmod 2)$. Рассматривая по отдельности случаи $\epsilon=0$ и $\epsilon=1 / 2$ и делая замену индексов, получим

$$
Z[A]=\frac{1}{|\eta(q)|^{2}} \sum_{(m, \bar{m}) \in \mathbb{Z}^{2}} e^{2 i \pi b(m-\bar{m})} q^{\frac{1}{2}(m-a)^{2}} \bar{q}^{\frac{1}{2}(\bar{m}-a)^{2}} .
$$

Наконец, выражение (3.6) может быть переписано через тета-функции Римана с характеристиками (см. приложение Е), что дает известный результат $[27,32,63]$

$$
Z\left[A_{a, b}\right]=\frac{1}{|\eta(q)|^{2}} \vartheta\left[\begin{array}{c}
a \\
-b
\end{array}\right](0, \tau) \overline{\vartheta\left[\begin{array}{c}
a \\
-\bar{b}
\end{array}\right](0, \tau)}
$$

Здесь мы предположили, что $b$ комплексно, а $a$ вешественно, поскольку химический потенциал отвечает чисто мнимому $b$. Это важно для сравнения бозонной и фермионной статистических сумм, выполненного в приложении В. Как мы увидим впоследствии, взаимодействуюший случай является прямым обобшением невзаимодействуюшего случая, если заменить тета-функцию подходящим обобщением.

\section{2. Калибровочные преобразования}

Строго говоря, предыдушее вычисление было выполнено при постоянном калибровочном поле. Зная свойства преобразований $Z_{\left[\epsilon, \epsilon^{\prime}\right]}[A]$ при обычных $(A \mapsto A+d \chi)$ и киральных $\left(A \mapsto A+d^{*} \lambda\right)$ калибровочных преобразованиях, мы получим $Z[A]$ для каждого калибровочного поля $A$, поскольку по теореме разложения Ходжа любая один-форма $A$ может быть разложена единственным образом как

$$
A=h+d \chi+d^{*} \lambda \text {. }
$$


Здесь $h$ - гармоническая один-форма или, что эквивалентно на торе, постоянная связность. Напомним, что калибровочные параметры $\chi$ и $\lambda$ - хорошо определенные функции на торе (с нулевой монодромией).

Рассмотрим сначала обычные калибровочные преобразования. Используя соотношение (D.9) (см. приложение D) и точность формы $d \chi$, мы получим

$$
S_{\wedge}[\varphi, A+d \chi]-S_{\wedge}[\varphi, A]=\frac{i}{\pi R} \int d \chi \wedge d \varphi=0
$$

Статистическая сумма точно калибровочно-инвариантна при обычных калибровочных преобразованиях.

Обратимся теперь к киральным калибровочным преобразованиям:

$$
A \rightarrow A+d^{*} \lambda
$$

где $d^{*} \lambda=\epsilon^{\mu \nu}\left(\partial_{\nu} \lambda\right) d x^{\mu}$. Элементарные вычисления дают ответ

$$
S_{\wedge}\left[\varphi, A+d^{*} \lambda\right]=S_{\wedge}\left[\varphi-\frac{i}{g R} \lambda, A\right]+\frac{1}{2 \pi} \int(d \lambda)^{2}+\frac{1}{\pi \alpha} \int A \wedge d \lambda
$$

Таким образом, статистическая сумма имеет следуюшие законы преобразования при обычных и киральных калибровочных преобразованиях:

$$
\begin{aligned}
Z[A+d \chi] & =Z[A] \\
Z\left[A+d^{*} \lambda\right] & =Z[A] \exp \left(-\frac{1}{2 \pi \alpha} \int(d \lambda)^{2}-\frac{1}{\pi \alpha} \int A \wedge d \lambda\right) .
\end{aligned}
$$

Важно отметить, что трансформационные свойства бозонных функциональных интегралов никак не зависят от граничных условий. Тождества (3.12) и (3.13) являются тождествами Уорда для КТП Люттингера.

\section{3. Некиральная бозонизация}

Здесь мы используем технику функционального интегрирования для установления связи между фермионными функциональными интегралами безмассовой модели Тирринга в спиновом секторе АА и соответствующим бозонным функциональным интегралом. Эта формула задает бозонизацию безмассовой модели Тирринга функциональным образом. Этот подход к бозонизации отличается от обычного, основанного на киральной бозонизации левых и правых мод в операторном формализме (см. [55, гл. 14]). Здесь обе киральности рассматриваются одновременно. По этой причине мы называем эту процедуру некиральной бозонизацией.

3.3.1. Формулировка результата. Введем следуюшие обозначения:

$$
\int_{\mathcal{C}} \mathcal{D}[\varphi]=\frac{1}{2} \sum_{\left(\epsilon, \epsilon^{\prime}\right) \in\{0,1 / 2\}}(-1)^{4 \epsilon \epsilon^{\prime}} \int_{\left[\epsilon, \epsilon^{\prime}\right]} \mathcal{D}_{R}[\varphi]
$$


где граничные условия в интеграле по путям в правой части равенства имеют вид

$$
\begin{aligned}
& \varphi(z+1) \equiv \varphi(z)+2 \pi \epsilon R(\bmod 2 \pi R), \\
& \varphi(z+\tau) \equiv \varphi(z)+2 \pi \epsilon^{\prime} R(\bmod 2 \pi R),
\end{aligned}
$$

a $\mathcal{D}_{R}[\varphi]$ обозначает меру интегрирования для бозонного поля, компактифицированного на окружность радиуса $R$ (см. приложение С). Мы покажем, что справедливо следующее равенство, связывающее статистические суммы:

$$
\int_{\mathrm{AA}} \mathcal{D}[\bar{\psi}, \psi] \exp \left\{-S_{L}[\bar{\psi}, \psi]+i \int A_{\mu} \bar{\psi} \gamma_{E}^{\mu} \psi\right\}=\int_{\mathcal{C}} \mathcal{D}[\varphi] e^{-S_{\wedge}[\varphi, A]}
$$

Здесь $S_{L}$ - действие безмассовой модели Тирринга, заданной лагранжианом (2.44), a $S_{\wedge}$ - действие (3.1) компактного бозонного поля, взаимодействующего с калибровочным полем посредством внешнего произведения. Эта формула описывает некиральную бозонизацию КТП Люттингера в присутствии калибровочного фонового поля. Фермионньй ток также может быть выражен через бозонное поле:

$$
\bar{\psi} \gamma_{\mathrm{E}}^{\mu} \psi=\frac{\epsilon^{\mu \nu} \partial_{\nu} \varphi}{\pi R}
$$

Как принято в КТП, это равенство справедливо для вставок внутри корреляционных функций.

3.3.2. Случай взаимодействия. Докажем теперь (3.16) в наиболее общем случае. Метод доказательства состоит в следуюшем: заметив, что это уравнение выполняется для однородного калибровочного фонового поля, мы сначала докажем его с помошью тождеств Уорда в теории Дирака в случае калибровочного фонового поля обшего вида. Затем случай взаимодействия будет проанализирован с помощью преобразования Хаббарда-Стратоновича. Докажем уравнение (3.16) для произвольного калибровочного поля: мы знаем закон преобразования фермионных статистических сумм как при киральных, так и при обычных калибровочных преобразованиях. Эти суммы инвариантны относительно последних, в то время как при киральных калибровочных преобразованиях возникают аномальные члены (см. уравнения (3.12) и (3.13)). Тем самым фермионные статистические суммы и бозонный функциональный интеграл, возникаюший в (3.16), имеют одни и те же свойства преобразований при обычных и киральных калибровочных преобразованиях и совпадают при постоянных калибровочных полях. Таким образом, если мы теперь применим теорему разложения Ходжа для один-формы на торе, эти функционалы совпадут всюду.

Введем теперь в рассмотрение член взаимодействия (2.43) типа взаимодействия модели Тирринга. Четырехфермионное взаимодействие полезно расцепить с помощью введения вспомогательного поля $b_{\mu}$ :

$$
\exp \left(-\frac{\kappa}{2} \int\left(\bar{\psi} \gamma_{\mathrm{E}}^{\mu} \psi\right)^{2}\right)=\int \mathcal{D}\left[b_{\mu}(x)\right] \exp \left(\frac{1}{2 \kappa} \int b(x)^{2}+i \int b_{\mu} \bar{\psi} \gamma_{\mathrm{E}}^{\mu} \psi\right)
$$


Таким образом, мы вернулись к предыдущему случаю в присутствии флуктуируюшего векторного потеншиала:

$$
\begin{aligned}
Z_{\mathrm{Lutt}}[A] & =\int_{\mathrm{AA}} \mathcal{D}[\bar{\psi}, \psi] \exp \left\{-S_{L}[\bar{\psi}, \psi]-\frac{\kappa}{2} \int\left(\bar{\psi} \gamma_{\mathrm{E}}^{\mu} \psi\right)^{2}+i \int A_{\mu} \bar{\psi} \gamma_{\mathrm{E}}^{\mu} \psi\right\}= \\
& =\int_{\mathrm{AA}} \mathcal{D}[\bar{\psi}, \psi] \int \mathcal{D}\left[b_{\mu}(x)\right] \exp \left\{-S_{L}[\bar{\psi}, \psi]-\frac{1}{2 \kappa} \int b(x)^{2}+i \int\left(b_{\mu}+A_{\mu}\right) \bar{\psi} \gamma_{\mathrm{E}}^{\mu} \psi\right\} .
\end{aligned}
$$

Применим теперь тождество (3.16), котороепока было доказано только в свободной теории и в присутствии флуктуирующего калибровочного потеншиала. Получим

$$
\begin{aligned}
Z_{\text {Lutt }}[A]= & \int_{\mathcal{C}} \mathcal{D}[\varphi] \int \mathcal{D}\left[b_{\mu}(x)\right] \exp \left\{-\frac{g}{2 \pi} \int(\partial \varphi)^{2}+\right. \\
& \left.+\frac{i}{\pi R} \int(A+b) \wedge d \varphi-\frac{1}{2 \kappa} \int(b(x))^{2}\right\} .
\end{aligned}
$$

Проинтегрировав по вспомогательному полю $b_{\mu}$, мы находим

$$
Z_{\mathrm{Lutt}}[A]=\int_{\mathcal{C}} \mathcal{D}[\varphi] \exp \left\{-\frac{g}{2 \pi}\left(1+\frac{\kappa}{\pi g R^{2}}\right) \int(\partial \varphi)^{2}+\frac{i}{\pi R} \int A \wedge d \varphi\right\} .
$$

Эффект взаимодействия состоит в изменении константы связи (или эквивалентно радиуса компактификации):

$$
\frac{g^{\prime}}{g}=1+\frac{\kappa}{\pi \alpha}
$$

Обсуждение нестабильности. Заметим, что теории со взаимодействием определены только при $\kappa>-\pi$. При $\kappa \leqslant-\pi$ бозонное действие перестает быть положительно-определенным. Такое ограничение появляется уже в операторном формализме. Формула (2.18) показывает, что для $\left|g_{2}\right| \geqslant\left|v_{\mathrm{F}}+g_{4}\right|$ преобразование Боголюбова перестает работать. Легко видеть, что спектр бозонного гамильтониана (2.15) при этом не ограничен снизу. Это отвечает нестабильности, которая проявляется на языке функционального интеграла вследствие равенства $\alpha^{2}=\left(v_{\mathrm{F}}+g_{4}-g_{2}\right) /\left(v_{\mathrm{F}}+g_{4}+g_{2}\right)$.

Попробуем понять происхождение этой нестабильности непосредственно в фермионном подходе. Напомним, что в массивной модели Тирринга Корепин [64] показал, что в режиме отталкивания $(-\pi<g<-\pi / 2)$ спектр включает связанные состояния. Возврашаясь к безмассовому случаю, когда $\kappa \rightarrow-\pi$, мы видим, что фермионы стремятся образовать конденсат связанных состояний и модель становится нестабильной при $\kappa=-\pi$. Однако вопрос, как вьплядит связь между этой нестабильностью (в массивном режиме) и нестабильностью бозонной теории, остается открытым.

Итак, возникновение таких нестабильностей естественно с точки зрения физики твердого тела. Действительно, они возникают, когда взаимодействия, измеряемые в подходящих единицах, становятся порядка скорости Ферми $v_{\mathrm{F}}$. В этом случае мы ожидаем, что электронная жидкость будет нестабильной (см., например, книгу Андерсона [3]). 


\section{4. Вычисление эффективного действия}

В этом разделе будет явно проделано интегрирование по полям материи модели Люттингера, и мы тем самым получим эффективное действие для векторного потенциала. С точки зрения теории поля это сводится к решению модели Швингера [65] на торе в выделенном спиновом секторе. Как будет показано в следующем разделе, эффективное действие является производяшим функционалом для корреляционных функций зарядовых и токовых плотностей.

Все вычисления проводятся в бозонной теории. После выделения вкладов нулевых мод векторного потенциала остается проделать прямое вычисление.

3.4.1. Выделение нулевых мод. Пусть $A=A_{\mu} d x^{\mu}$ - векторный потенциал. Теория Ходжа предсказывает, что он может быть разложен единственным образом как $A=h_{A}+d \chi+d^{*} \lambda$, где $h_{A}-$ однородный векторный потенциал. После этого получим

$$
Z[A]=Z\left[h_{A}\right] \exp \left(-\frac{1}{2 \pi \alpha} \int(d \lambda)^{2}\right),
$$

где мы использовали тождество Уорда (3.13).

3.4.2. Явное вычисление. Вычислим теперь $\lambda$ в терминах векторного потенциала $A$. Введем разложение $B=A-h_{A}$ таким образом, чтобы каждая компонента $B$ имела нулевое среднее на торе. Один-форма $d^{*} \lambda-B$ точна, и тем самым $d d^{*} \lambda=d B$. Однако, поскольку $d d^{*} \lambda=-(\Delta \lambda)^{*}$, скалярная кривизна $F_{B}$ формы $B$ имеет вид

$$
F_{B}=\Delta \lambda
$$

Лапласиан обратим на ядре нормированного интеграла по тору:

$$
\int_{N}: f \mapsto \frac{1}{\mathcal{A}} \int f
$$

где $\mathcal{A}$ - полная площадь тора. Если надо найти обратный оператор к лапласиану, следует решить уравнение Грина:

$$
\Delta_{x} G(x, y)=\delta(x-y)-\frac{1}{\mathcal{A}}
$$

Различные решения этого уравнения отличаются на константу, которая не важна вследствие того, что функция Грина всегда действует на функцию с нулевым средним. Обозначим через $\Delta^{-1}$ обратный лапласиан на этом векторном пространстве. Его явное выражение приведено в приложении Е. Обрашая уравнение (3.23) и вводя комплексные координаты, мы получим

$$
Z[A]=Z\left[h_{A}\right] \exp \left(K\left[B_{z}, B_{\bar{z}}\right]\right)
$$

где

$$
\begin{aligned}
K\left[B_{z}, B_{\bar{z}}\right]= & \frac{2}{\pi \alpha} \int d^{2} z d^{2} \xi\left(B_{z} B_{\xi}\left(\partial_{\bar{z}}^{2} \Delta^{-1}\right)(z-\xi)+B_{\bar{z}} B_{\bar{\xi}}\left(\partial_{z}^{2} \Delta^{-1}\right)(z-\xi)\right)- \\
& -\frac{1}{\pi \alpha} \int B_{z} B_{\bar{z}} d^{2} z
\end{aligned}
$$


Как упомянуто в приложении Е, обе вторые производные $\partial_{z}^{2} \Delta^{-1}$ и $\partial_{\bar{z}}^{2} \Delta^{-1}$ понимаются в смысле обобщенных функций (см. уравнение (Е.16) приложения Е). Эти обобщенные функции выражаются через ६-функцию Вейерштрасса по формуле (Е.22) и ее комплексно-сопряженную. Эта тонкость важна при вычислении корреляционных функций плотность-плотность в присутствии внешнего потенциала (см. раздел 5.1).

Другие авторы проводят выгисления на плоскости именно в пределе нулевой температуры и в термодинамическом пределе. В этом случае имеются два отличия от результатов, полученных в данной статье: во-первых, вклады нулевых мод векторного потенциала $A$ не рассматриваются. Затем выражение для функции Грина оказывается более простым. Тем не менее идея подхода та же, и явное интегрирование по полям материи уже давно стало весьма полезным приемом, используемым в этих двумерных теориях поля [65].

\section{4. ЖЛ С БЕСПОРЯДКОМ ПРИ РАССЕЯНИИ ВПЕРЕД}

Формализм, развитый в предыдуших разделах, будет теперь применен к исследованию ЖЛ в присутствии магнитного потока и при наличии весьма особого вида беспорядка - гауссова внешнего потенциала, взаимодействуюшего с фермионными плотностями $\rho(\sigma)$, как показано в уравнении (2.8). Эффекты рассеяния назад и, следовательно, локализационные эффекты в этой упрощенной модели отсутствуют. Это, конечно, существенный недостаток нашего исследования, но учет рассеяния назад невозможен без подробного исследования взаимодействия типа синус-Гордон, что выходит далеко за рамки данной работы. В случае, если эти взаимодействия становятся сушественными, мы теряем свойства конформности и должны использовать другие методы. Описание беспорядка, которое более пригодно для систем типа квазиодномерных проводников, могло бы включать в рассмотрение примеси, расположенные вдоль цепей, и, следовательно, рассеяние назад. В семидесятые годы русской школой были развиты различные подходы, такие как диаграммная техника Березинского (см., например, [66-73]) для описания беспорядка подобного типа в отсутствие электрон-электронного взаимодействия. Совместные эффекты беспорядка и взаимодействия были исследованы в [44] с помошью техники ренормализационной группы. В приведенных выше работах наличие беспорядка порож дает либо массивные фазы, либо безмассовые фазы с сингулярной низкоэнергетической плотностью состояний. Очевидно, что в обоих случаях получаемая разупорядоченная система не конформна и проблема ее исследования с помошью методов, аналогичных использованным в данной статье, остается открытой.

Тем не менее в этом подходе корреляционные функции и их средние по указанному модельному беспорядку могут быть вычислены без применения метода реплик. Метод, использованный здесь, основывается на свойствах преобразований лагранжиана при киральных калибровочных преобразованиях. Он был применен Бернаром $[74,75]$ при решении модели со случайным векторным потеншиалом. Как будет показано в разделе 5 , свойства переноса не зависят от модельного беспорядка. Этого следовало ожидать, поскольку в модели отсутствует локализация. Мы вычислим также удельную теплоемкость ЖЛ в режиме, при котором температура много больше, чем межуровневое расстояние $2 \pi v_{S} / L$, и покажем, что она не зависит от простого модельного беспорядка, рассматриваемого в данной работе. 


\section{1. Общая постановка задачи и обозначения}

Введем случайный классический потенциал $V(\sigma)$ и рассмотрим постоянное магнитное поле с магнитным потоком $\Phi$ через кольцо, число $\chi=\Phi / \Phi_{0}$ обозначает количество квантов потока, где $\Phi_{0}=2 \pi / e-$ квант потока. Причина, по которой мы рассматриваем одновременно взаимодействие с внешним потенциалом и магнитным полем, состоит в том, что, как мы увидим впоследствии, оба взаимодействия могут быть рассмотрены в рамках одного и того же подхода, поскольку они связаны преобразованиями дуальности.

Выберем гауссову функцию распределения случайного потенциала:

$$
P[V(\sigma)]=\exp \left(-\frac{1}{2 \gamma} \int_{0}^{L} d \sigma V(\sigma)^{2}\right)
$$

Температурные и квантовые средние по отношению к системе Люттингера обозначаются через $\langle\ldots\rangle$ :

$$
\langle O[\bar{\psi}, \psi]\rangle_{[\chi, V(\sigma)]}=\frac{\int \mathcal{D}[\bar{\psi}, \psi] e^{-S[\bar{\psi}, \psi, V, \chi]} O[\bar{\psi}, \psi]}{\int \mathcal{D}[\bar{\psi}, \psi] e^{-S[\bar{\psi}, \psi, V, \chi]}}
$$

где фермионные поля принадлежат сектору АА. Обозначим через $\bar{X}$ среднее от $X$ по модельному беспорядку и будем исследовать корреляционные функции вида

$$
\overline{\prod_{k=1}^{N}\left\langle O_{k}[\bar{\psi}, \psi]\right\rangle}=\int \mathcal{D}[V] \exp \left\{-\frac{1}{2 \gamma} \int_{0}^{L} d \sigma V(\sigma)^{2}\right\} \prod_{k=1}^{N}\left\langle O_{k}[\bar{\psi}, \psi]\right\rangle_{[\chi, V(\sigma)]} .
$$

Как будет показано ниже, эти корреляционные функции могут быть явно вычислены без использования метода реплик. Для этого мы, во-первых, бозонизуем систему с помощью приема, описанного в предыдуших разделах, а затем используем квадратичность действия по бозонному полю и случайному потенциалу. В этом месте выбор гауссова распределения для случайного потенциала играет определяющую роль.

Вклад беспорядка и магнитного поля в фейнмановские веса. Статистический вес, ассоциированный с беспорядком и магнитным полем, имеет вид

$$
W[\bar{\psi}, \psi]=\exp \left(i \int_{0}^{\beta} d u \int_{0}^{L} d \sigma\left[\frac{e v_{f} \Phi}{L} \bar{\psi} \gamma^{1} \psi+i e V(\sigma) \bar{\psi} \gamma^{0} \psi\right]\right)
$$

Его полезно переписать как $\exp \left(i \int j \cdot A\right)$, где $j^{\mu}=\bar{\psi} \gamma^{\mu} \psi$, а $A_{0}=i e V(\sigma)$ и $A_{1}=e v_{f} \Phi / L$.

\section{2. Средние по беспорядку от корреляционных функций}

Выведем теперь общую формулу для вычисления средних по беспорядку от любого произведения корреляционных функций. Расщепим потенциал $V(\sigma)$ на его среднюю и флуктуируюшую части:

$$
V(\sigma)=\mathcal{V}(\sigma)+\frac{Q}{L},
$$


и введем функцию $\eta(\sigma)$, определенную как

$$
\eta(\sigma)=\int_{0}^{\sigma} \mathcal{V}(x) d x
$$

Мы предполагаем, что наблюдаемая величина $O$ выражается через функционал бозонного поля. В этом случае мы вводим следуюшие обозначения:

$$
Z_{O}[\chi, V(\sigma)]=\int_{\mathcal{C}} \mathcal{D}[\varphi] e^{-S[\varphi, A]} O[\varphi]
$$

Идея состоит в том, чтобы включить флуктуирующую часть $\eta(\sigma)$ потенциала в переопределение поля $\varphi$. На языке КТП это абелева версия тождества Полякова-Вигмана [76]. Простые выкладки дают ответ:

$$
Z_{O}[\chi, V(\sigma)]=\exp \left\{\frac{e^{2} \beta}{2 \pi g R^{2}} \int_{0}^{L} d \sigma \eta^{\prime}(\sigma)^{2}\right\} Z_{O[\varphi+e \eta / g R]}[\chi, Q / L]
$$

Здесь поле $\varphi$ было сдвинуто на $е \eta / g R$. Это сдвиг не меняет граничных условий бозонного поля, поскольку $\eta(0)=\eta(L)=0$. Так как экспоненциальный множитель в правой части уравнения (4.8) не зависит от наблюдаемой $O$, он сокрашается при взятии средних от $O$ :

$$
\langle O[\varphi]\rangle_{[\chi, V(\sigma)]}=\left\langle O\left[\varphi+\frac{e}{g R} \eta\right]\right\rangle_{[\chi, Q / L]} .
$$

Используя эти уравнения, можно вьгислить средние по модельному беспорядку:

$$
\begin{aligned}
& \overline{\prod_{k=0}^{N}\left\langle O_{k}[\phi]\right\rangle}=\int_{-\infty}^{+\infty} \frac{d Q}{\sqrt{2 \pi \gamma L}} e^{-\frac{Q^{2}}{2 \gamma L} \times} \\
& \quad \times \int \mathcal{D}[\eta(\sigma)] \delta(\eta(0)) \exp \left\{-\frac{1}{2 \gamma} \int_{0}^{L} d \sigma \eta^{\prime}(\sigma)^{2}\right\} \prod_{k=0}^{N}\left\langle O_{k}\left[\phi+\frac{e}{g R} \eta\right]\right\rangle_{[\chi, Q / L]} .
\end{aligned}
$$

Таким образом, мы достигли своей цели: средние по модельному беспорядку вычислены без использования метода реплик. В последуюших разделах мы предъявим явные примеры корреляционных функций, усредненных по модельному беспорядку. Однако перед этим мы обратимся к проблеме вычисления среднего по беспорядку от свободной энергии.

\section{3. Средняя свободная энергия, теплоемкость}

Представим сначала статистическую сумму КТП Люттингера (2.57) в виде

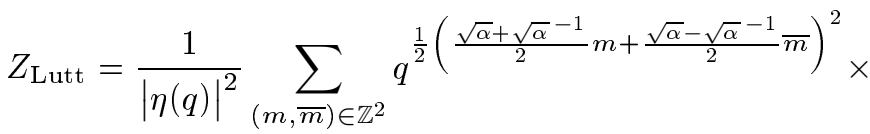

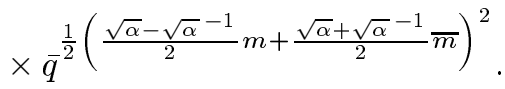

2 Теоретическая и математическая физика, т. 117, № 1, 1998 г. 
Нам интересно поведение статистической суммы в пределе $\tau=i \beta v_{S} / L \rightarrow 0^{+}$. Физически это означает, что температура много больше межуровневого расстояния. Поэтому число возбужденных уровней очень велико. Напомним, что реальные системы могут быть описаны эффективной теорией Люттингера не при всех энергиях. Пусть $E^{*}$ обозначает масштаб энергий, при котором эффективное описание через взаимодействуюшую безмассовую модель Тирринга перестает работать. Мы будем предполагать, что $E^{*} \gg 2 \pi v_{S} / L$. Этот энергетический масштаб $E^{*}$ может появляться из-за кривизны, нелокальности взаимодействия и т.д. Наш анализ будет работать в области температур, для которых

$$
\frac{2 \pi v_{S}}{L} \ll k_{\mathrm{B}} T \ll E^{*} .
$$

В переменных $x=2 \pi v_{S} \beta / L$ нужный предел будет $x \rightarrow 0^{+}$. В отсутствие подкрутки в пространственном направлении (другими словами, когда $\theta=0$ в (2.32)) мы получим $q=\bar{q}=e^{-x}$. В переменных $x$ статистическая сумма имеет вид

$$
\begin{aligned}
Z_{\text {Lutt }}(\alpha,[A])= & \frac{1}{\eta(i x / 2 \pi)^{2}} \sum_{(n, \bar{n}) \in \mathbb{Z}^{2}} \exp \left(-\frac{x}{4}\left(\alpha(n+\bar{n})^{2}+\alpha^{-1}(n-\bar{n}-2 a)^{2}\right)\right) \times \\
& \times \exp (2 i \pi b(n+\bar{n})) .
\end{aligned}
$$

Ее асимптотическое поведение при $x \rightarrow 0^{+}$получается из асимптотики функции Дедекинда в пределе $\tau \rightarrow i 0^{+}$. Подставляя $b=i \beta v_{S} Q / 2 \pi L$, мы получим

$$
\ln \left(Z_{\text {Lutt }}(\alpha,[A])\right) \sim \frac{\pi L}{6 \beta v_{S}}+\frac{\beta v_{S} Q^{2}}{2 \pi \alpha L} .
$$

Перед тем как усреднять по скрученному модельному беспорядку, отметим, что эффект присутствия магнитного поля и электрического потенциала сводится к уменьшению свободной энергии на величину $v_{f} Q^{2} / 2 \pi \alpha L$, которая не зависит ни от температуры, ни от магнитного поля. В частности, удельная теплоемкость не зависит ни от электрического потенциала, ни от магнитного поля. Разумеется, это заключение остается справедливым после усреднения по беспорядку.

\section{5. КОРРЕЛЯЦИОННЫЕ ФУНКЦИИ ЗАРЯД-ЗАРЯД И ТОК-ТОК}

Приложим развитый вьше формализм к вычислению корреляций плотностей заряда и тока в КТП Люттингера. Вычисления будут основываться на вычислении производящего функционала для корреляторов зарядовой и токовой плотностей. Нас будут интересовать корреляционные функции в режиме сохранения полного заряда системы. Этот полный заряд обозначим через $q$. Усреднение по $q$ сводится к рассмотрению набора из многих изолированных колец Люттингера, из которых некоторые содержат нечетное число носителей заряда, в то время как остальные - четное число. Опять-таки наша задача - не описать сколько-нибудь реалистичным образом физику непрерывных токов в кольцах промежуточного размера, которая для своего описания требует рассмотрения многоканальных процессов, влияния примесей, спинов и т.д. и которая тем самым выходит далеко за рамки обсуждаемого формализма, а продемонстрировать, что 
формализм, исследованный в предыдущих разделах, позволяет вычислить такие физические величины, как постоянные токи в кольце Люттингера. Для большей общности вычислим сначала производяший функционал для $n$-точечных коррелящионных функций плотностей и токов.

\section{1. Корреляторы плотностей заряда и тока}

5.1.1. Формулировка результатов. Производящий функционал для корреляторов плотности заряда имеет вид

$$
W_{[V(\sigma), \chi, q]}^{(0)}[b(z)]=\left\langle\exp \left(\int d \sigma d t b(\sigma, t) \rho(\sigma, t)\right)\right\rangle .
$$

Его явное выражение, которое может быть получено из эффективного действия (3.25),

$$
W^{(0)}[V(\sigma), \chi, q]=\exp \left(L_{0}[V(\sigma), b(z)]+F_{0}[b(z)]\right),
$$

включает в себя линейный член

$$
L_{0}[V(\sigma), b(z)]=\int d \sigma d u\left(\frac{q}{L}-\frac{1}{\pi \alpha} \mathcal{V}(\sigma)\right) b(\sigma, u)
$$

и квадратичный член

$$
F_{0}[b(z)]=\exp \left(\frac{1}{4 \pi \alpha} \int d^{2} z d^{2} \xi b(z) b(\xi) G_{0}(z-\xi)\right) .
$$

Ядро $G_{0}$ выражается следующим образом:

$$
G_{0}(z-\xi)=-\Re\left(\frac{\wp(z-\xi)}{\pi}\right)+\delta(z-\xi) .
$$

Разумеется, вклад функции Вейерштрасса понимается как регуляризованная обобщенная функция подобно тому, как сделано в формуле (Е.22) приложения Е. Детали вычисления будут приведены в следуюшем пункте.

Производящий функционал корреляторов токов определяется как

$$
W_{[V(\sigma), \chi, q]}^{(1)}[c(z)]=\left\langle\exp \left(\int d \sigma d t c(\sigma, t) j(\sigma, t)\right)\right\rangle .
$$

Он может быть явно вычислен, что дает

$$
W_{[V(\sigma), \chi, q]}^{(1)}[c(z)]=\frac{Z_{q}\left[0, \chi+\frac{1}{2 \pi \beta} \int c(z) d^{2} z\right]}{Z_{q}[0, \chi]} \exp \left(F_{1}[\tilde{c}(z)]\right),
$$

где

$$
\tilde{c}(z)=c(z)-\frac{1}{\mathcal{A}} \int c(\xi) d^{2} \xi .
$$

Квадратичный вклад $F_{1}$ имеет вид

$$
F_{1}[\tilde{c}(z)]=-\frac{1}{4 \pi \alpha} \int d^{2} z d^{2} \xi \tilde{c}(z) \tilde{c}(\xi)\left(\Re\left(\frac{\wp(z-\xi)}{\pi}\right)+\delta(z-\xi)\right) .
$$


5.1.2. Детали вычисления. Покажем, как получить формулы (5.2)-(5.5). Начнем со следуюшего потенциала: $A_{\sigma}=0$ и $A_{u}=i(\mathcal{V}(\sigma)+b(\sigma, u))$. Из этого получим, что $B_{z}=-B_{\bar{z}}=(\mathcal{V}-\tilde{b}) / 2$, где

$$
\tilde{b}(z)=b(z)-\frac{1}{\mathcal{A}} \int b(\xi) d^{2} \xi .
$$

Подставляя эти выражения в формулу (3.26), мы получим три различных вклада:

$$
\begin{aligned}
& \frac{1}{2 \pi \alpha} \int\left(\left(\partial^{2}+\bar{\partial}^{2}\right) \Delta^{-1}+\frac{\delta}{2}\right)(z-\xi) \tilde{b}(z) \tilde{b}(\xi) d^{2} z d^{2} \xi \\
& \frac{1}{2 \pi \alpha} \int\left(\left(\partial^{2}+\bar{\partial}^{2}\right) \Delta^{-1}+\frac{\delta}{2}\right)(z-\xi) \mathcal{V}\left(\sigma_{z}\right) \mathcal{V}\left(\sigma_{\xi}\right) d^{2} z d^{2} \xi \\
& \frac{1}{\pi \alpha} \int\left(\left(\partial^{2}+\bar{\partial}^{2}\right) \Delta^{-1}+\frac{\delta}{2}\right)(z-\xi) \tilde{b}(z) \mathcal{V}\left(\sigma_{\xi}\right) d^{2} z d^{2} \xi
\end{aligned}
$$

Первый член сразу дает $F_{0}[b(z)]$, а второй сокрашается при нормировании на статистическую сумму. Только третий член заслуживает внимания. Главное наблюдение здесь - это то, что производные $\Delta^{-1}$ должны пониматься как производные обобщенной функции (объяснение дается в приложении Е.2). Для простоты при вычислениях использовалась решетка $\mathbb{Z} \oplus \tau \mathbb{Z}$. Пусть $f$ будет периодической функцией с периодом 1 по вещественной переменной. Зададим $\mathbb{Z} \oplus \tau \mathbb{Z}$-периодическую функцию $f_{1}$ формулой $f_{1}(z)=f(\Re(z))$. Теперь надо найти обобщенную функцию $T$ такую, что

$$
T \cdot f=\partial_{z}^{2}\left[\Delta^{-1}\right] \cdot f_{1}
$$

Заметив, что $f_{1}$ зависит только от $\Re(z)$, мы получим $\partial_{z} f_{1}=\left(\partial_{z}+\partial_{\bar{z}}\right) f_{1}$, и, таким образом, $\partial_{z}^{2}\left[\Delta^{-1}\right] \cdot f_{1}=\partial_{\bar{z}} \partial_{z}\left[\Delta^{-1}\right] \cdot f_{1}$. В этом случае применение формулы (Е.23) дает

$$
T \cdot f=\partial_{z}^{2}\left[\Delta^{-1}\right] \cdot f_{1}=\frac{1}{4}(\delta-1) \cdot f
$$

где обобщенные функции теперь действуют на $f$-функцию вешественного переменного. Другими словами, для пробной функции $f$ с нулевым средним мы получим

$$
\int f\left(\sigma_{z}\right) g(\xi) \frac{\wp(z-\xi)}{\pi} d^{2} z d^{2} \xi=\int f(\sigma) g(\sigma, u) d \sigma d u
$$

Тогда вклад $\mathcal{V} \times b$ задается $\mathcal{V}$-зависимой частью $L_{0}[V, b]$, а второй вклад имеет вид

$$
\exp \left(\frac{1}{2 \pi \alpha} \int d^{2} z \mathcal{V}\left(\sigma_{z}\right)^{2}\right)
$$

Этот результат может быть выведен с помошью тождества Уорда (3.13).

Данное вычисление требует некоторых пояснений. Оно показывает, что в правильной интерпретации корреляционные функции КТП, будучи обобщенными функциями, позволяют установить ультрафиолетовые свойства модели исходя из ее ультрафиолетового эффективного описания. Более точно, спаривание $b$ и потенциала, которое описывает зарядовую восприимчивость по отношению к внешнему потенциалу, оказывается 
локальным.м в подходе КТП. Тем не менее это спаривание может быть получено из эффективной теории при больщих расстояниях, поскольку оно основано на симметрийных свойствах системы, т.е. на ее трансформационных свойствах относительно киральных калибровочных преобразований, которые полагаются не зависимыми от описания системы.

Уравнения (5.7) и (5.8) доказываются аналогично. Надо вычислить $K[C+D, C-D]$, где $C=\tilde{c}(z) / 2$ и $D=\mathcal{V}(\sigma) / 2$. При этом получатся два члена, первый из которых имеет вид

$$
F_{1}[c(z)]+\frac{1}{2 \pi \alpha} \int d^{2} z \mathcal{V}\left(\sigma_{z}\right)^{2}
$$

а второй содержит перекрестный член типа $\tilde{c} \times \mathcal{V}$ :

$$
\frac{i}{2 \pi \alpha} \lim _{\varepsilon \rightarrow 0^{+}} \int_{|z-\xi|>\varepsilon} d^{2} z d^{2} \xi \tilde{c}(z) \mathcal{V}\left(\sigma_{\xi}\right) \Im\left(\frac{\wp(z-\xi)}{\pi}\right) .
$$

Далее заметим, что в уравнении (5.11) при подстановке мнимой части ю обращается в нуль. Таким образом, перекрестный член исчезает.

\section{2. Восприимчивость плотности к внешнему потенциалу}

5.2.1. Изменения плотности, вызванные внешним потенциалом. Дифференцируя один раз по $b(\sigma, t)$, мы получим среднюю плотность в присутствии потенциала $V(\sigma)$ :

$$
\rho_{\mathrm{av}}(\sigma)=\frac{q}{L}-\frac{1}{\pi v_{N}}\left(V(\sigma)-\frac{1}{L} \int_{0}^{L} V(\sigma) d \sigma\right) .
$$

Первый член в (5.12) обеспечивает сохранение заряда, причем полный заряд равен $q$. Функция отклика на внешний потенциал, таким образом, чисто локальна. Такой результат следует ожидать, поскольку известен отклик плотности для невзаимодействующих нерелятивистских фермионов в одномерном случае (см. приложение F, где приведены вычисления в этом пределе). При переходе к локальным взаимодействиям в ЖЛ мы должны лишь заменить скорость Ферми $v_{\mathrm{F}}$ скоростью заряда $v_{N}$, определенной в уравнении (2.24) через параметры взаимодействия. Этот результат, вполне возможно, стоило ожидать с самого начала, если принять во внимание физические основы модели.

5.2.2. Корреляционные функции. Связный двухточечный коррелятор плотностей сингулярен при совпадаюших временах, т.к. содержит дельта-функцию по времени u пространству. Интересно прояснить смысл этой сингулярности при малых расстояниях. Соответствующий объект возникает в системе нерелятивистских свободных фермионов на окружности, приведенной в приложении F. Это выгисление с делано для простоты при нулевой температуре, но оно может быть легко продолжено на любые конечные температуры. В нерелятивистской модели море Ферми имеет конечную глубину $k_{\mathrm{F}}$ и корреляции плотность-плотность имеют максимум, размазанньй по интервалу ширины $1 / k_{\mathrm{F}}$. В двухточечных корреляционных функциях при малых расстояниях появляются колебания вида $\sin ^{2}\left(k_{\mathrm{F}} \sigma\right)$, которые omcymcmвуют в нашем вычислении КТП. Эти два явных расхождения с результатами КТП могут быть проинтерпретированы следующим образом: во-первых, если мы рассматриваем расстояния сушественно меньше размера 


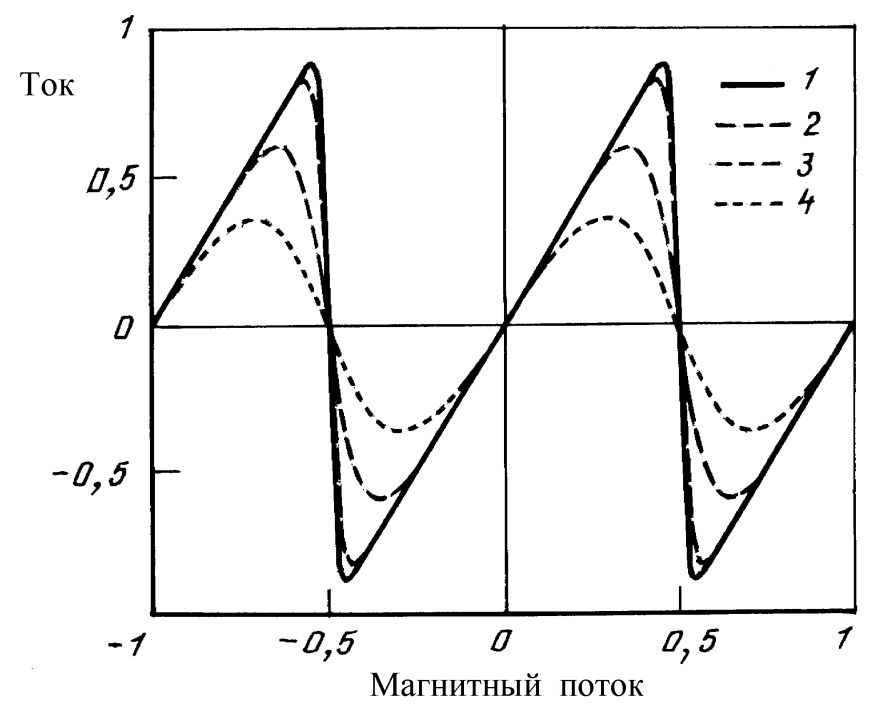

круга $L$, но больше “микроскопической” шкалы длин $1 / k_{\mathrm{F}}$, пик при малых расстояниях обратится в дельта-функцию с коэффициентом, пропорциональным $k_{\mathrm{F}}$ (она расходится в инфракрасном пределе). Это отвечает двойной дельта-функции, возникающей в конформном подходе. Во-вторых, усредняя $\sin ^{2}\left(k_{\mathrm{F}} \sigma\right)$ по расстояниям, гораздо большим чем $k_{\mathrm{F}}^{-1}$, мы получим $1 / 2$, т.е. результат, совпадающий с результатом конформного подхода.

Наконец, никакой из “сценариев” типа ортогонального не работает для корреляций плотность-плотность, что отличает этот случай от случая фермионных корреляций, как мы покажем ниже. Причина состоит в том, что хотя операторы плотности определены в терминах киральных фермионов, эти операторы являются составным.и. Априори также очевидно, что никакого поведения типа ортогональной модели не ожидается, поскольку при интегрировании $n$-точечной корреляционной функции по всем координатам мы получим неисчезающий член даже в термодинамическом пределе, что не допускает сушествования экспоненциально малых коэффициентов, стояших при указанных членах.

В секторе с фиксированным зарядом производящий функционал корреляций плотности гауссов (см. уравнение (5.2)). То же самое верно для флуктуаций плотности тока. С точностью до члена, ассоциированного с постоянным током, функционал (5.7) гауссов. Это следует из того, что фермионная плотность может рассматриваться как "координата" для этих квантовых теорий поля. Тогда плотность тока сопряжена этой координате. В терминах таких координат КТП Люттингера становится свободной, тем самым объясняя гауссову структуру этих функционалов.

\section{3. Постоянные токи в кольце Люттингера}

Как было указано многими авторами и обнаружено экспериментально, в кольцах среднего размера в присутствии магнитного потока появляются постоянные токи кван- 
тового происхождения (см., например, $[77,78])$. В ЖЛ эти токи могут быть точно вычислены при конечной температуре. Они допускают "универсальное" выражение в том смысле, что вся зависимость от взаимодействия заключена в параметрах Люттингера $\alpha$ и $v_{S}$, как и следует ожидать, если исходить из общих принципов, изложенных во введении.

Постоянный ток в изолированной системе заряда $q$ пропорционален производной свободной энергии по магнитному потоку:

$$
I[V(\sigma), \chi, q]=\left(\frac{\partial F}{\partial \Phi}\right)=\frac{e}{h}\left(\frac{\partial F}{\partial \chi}\right) .
$$

Прямое вычисление дает ответ:

$$
I[V(\sigma), \chi, q]=-\frac{e}{\beta h} \frac{\partial}{\partial \chi}\left(\log \left(\sum_{n \in \mathbb{Z}} \exp \left(-\frac{x}{\alpha}\left(\frac{q}{2}+\chi+n\right)^{2}\right)\right)\right) .
$$

В пределе нулевой температуры, это, очевидно, сводится к знаменитой “пилообразной" кривой. Максимальное значение равно $\pi e v_{J} / L$. На рисунке показаны вариации постоянных токов (в единицах измерения $2 \pi e v_{\mathrm{F}} / \alpha L$ ) вдоль кольца Люттингера при $q=0$ как функции потока внутри кольца (в единицах квантов потока) при различных значениях множителя $x / \alpha$ : кривая $1-50$; кривая 2 - 30; кривая $3-10$; кривая $4-5$. При уменьшении этого отношения "пилообразная" кривая становится все более сглаженной. Токи периодичны по магнитному потоку с периодом, равным кванту потока, что также видно на рисунке. Хотя мы и утверждаем, что ЖЛ далека от того, чтобы совершенно правильным образом смоделировать кольца среднего размера, эта периодичность с периодом кванта квантового потока была обнаружена экспериментально для отдельного кольца (см. [78]). Эти токи также периодичны по полному заряду $q$, но уже с периодом, равным двум квантам потока, как и ожидалось. Если усреднить по четности зарядов, то ток будет периодичен с периодом, равным половине кванта потока. Эта ситуация аналогична экспериментальной реализации большого ансамбля колец среднего размера, в которой наблюдались токи, имевшие такой половинный период [77]. Наконец, токи экспоненциально спадают, когда температура становится больше расстояния между уровнями $2 \pi v_{S} / L$, что следует также и из физических соображений.

\section{6. КОРРЕЛЯЦИОННЫЕ ФУНКЦИИ ВЕРШИННЫХ ОПЕРАТОРОВ}

В этом разделе мы вычисляем корреляционные функции вершинных операторов. Напомним, что в свободной бозонной КТП $\widehat{U(1)}$-примарные поля находятся во взаимно однозначном соответствии с киральными операторами. Они составляют конформные блоки КТП Люттингера. Как мы увидим в разделе 6.4 , перенормированный фермион в теории со взаимодействием отвечает одному из этих вершинных операторов.

Отождествив эффективную теорию краевых возмущений ДКЭХ-жидкости на цилиндре с одной из КТП Люттингера (см. раздел 7), мы увидим, что физические фермионы, локализованные на одном крае образца Холла, рождаются и уничтожаются специальными вершинными операторами. Тем самым вычисления, представленные в этом разделе, дают выход на физически важные корреляционные функции. 
Начнем с определения вершинных операторов, которое хорошо подходит для вычислений в методе функционального интеграла. Затем мы выведем корреляционные функции на сфере (это просто) и на торе. Наконец, мы обсудим возможное отождествление теории фермиона Люттингера и эффектов модельного беспорядка.

\section{1. Вершинные операторы}

Вместо того чтобы рассматривать вершинные операторы сами по себе, нас будут интересовать их произведения специального вида. Более точно, мы будем вычислять объекты типа средних от

$$
\prod_{k=1}^{n} \exp \left(\frac{i e_{k}}{R} \int_{C_{k}} d \varphi+\frac{e_{k}^{*}}{R} \int_{C_{k}} d^{*} \varphi\right) .
$$

Параметры $\left(e_{k}, e_{k}^{*}\right)_{k}$ - фиксированные вешественные числа, а $C_{k}$ - некоторые открытые ориентированные кривые, начинающиеся в точке $a_{k}$ и заканчивающиеся в $b_{k}$. Конечно, величины (6.1) должны быть перенормированы, что и будет объяснено в разделах, приведенных далее. Тем не менее интересно сначала выяснить значение таких величин.

Первый интеграл в (6.1) задает произведение обычных вершинных операторов, поскольку из него сразу получаются комбинации

$$
\exp \left(\frac{i e_{k}\left(\varphi\left(b_{k}\right)-\varphi\left(a_{k}\right)\right)}{R}\right)
$$

Чтобы понять физический смысл второго члена в (6.1), мы выберем локальные координаты около точки $x$ на кривой $C_{k}$. Пространственная координата $l_{x}$ выбирается локально вдоль кривой $C_{k}$, а координата мнимого времени $u_{x}$ - перпендикулярно к кривой $C_{k}$ в точке $x$. Тогда локальный вклад во второй интеграл имеет вид

$$
-\left(\partial_{u_{x}} \varphi\right)\left(l_{x}, u_{x}\right) d l_{x}
$$

Однако производная по мнимому времени пропорциональна импульсу $\Pi_{\varphi}$, сопряженному координате $\varphi$ :

$$
\Pi_{\varphi}=\frac{g}{\pi} \partial_{t} \varphi
$$

Таким образом, если обозначить через $\Pi_{C_{k}}$ импульс на кривой $C_{k}$, то получим

$$
\exp \left(\frac{e_{k}^{*}}{R} \int_{C_{k}} d^{*} \varphi\right)=\exp \left(-i \frac{\pi e_{k}^{*}}{g R} \Pi_{C_{k}}\right) .
$$

Второй интеграл в выражении (6.1), таким образом, отвечает введению дефекта вдоль кривой $C_{k}$, который является оператором беспорядка.

В этой интерпретации мы сразу видим, что как $e_{k}$, так и $e_{k}^{*}$ квантуются. Поскольку $\varphi$ компактифишируется на радиусе $R$, мы сразу получим $e_{k} \in \mathbb{Z}$. За счет беспорядка возникает скачок высоты $\pi e_{k}^{*} / g R$ для поля $\varphi$ вдоль кривой $C_{k}$. Тогда, накладывая условие, что этот сдвиг пропорционален $2 \pi R$, мы получим второе условие квантования: $e_{k}^{*} \in 2 \alpha \mathbb{Z}$. В действительности это не что иное, как два условия квантования обычной бозонной теории в модулярно-инвариантном секторе. Чтобы это понять, вычислим сначала корреляционные функции на сфере. 


\section{2. Корреляционные функции на сфере}

Вычислим двухточечную корреляционную функцию на римановой сфере:

$$
\frac{1}{Z_{\mathbb{P}_{1}(\mathbb{C})}} \int \mathcal{D}[\varphi] \exp \left(\frac{i e}{R} \int_{C} d \varphi+\frac{e^{*}}{R} \int_{C} d^{*} \varphi-\frac{g}{2 \pi} \int(d \varphi)^{2}\right),
$$

где $C$ начинается в точке $a$ и заканчивается в $b$. Этот гауссов интеграл может быть вычислен методом седловой точки. В дальнейшем $\delta_{C}$ будет обозначать обобшенную дельта-функцию, локализованную на ориентированной кривой $C$, а $\partial_{n} \delta_{C}-$ ее производную по отношению к координате, нормальной к сфере. Уравнение седловой точки имеет вид

$$
\Delta \varphi=\frac{i \pi e}{g R}\left(\delta_{a}-\delta_{b}\right)-\frac{\pi e^{*}}{g R} \partial_{n} \delta_{C}
$$

Как и ожидалось, при пересечении кривой $C$ в прямом направлении по отношению к ориентации $C$ поле $\varphi$ уменњшается на $\pi e^{*} / g R$. С точностью до аддитивной постоянной решение этого уравнения может быть выражено через фиксированную ветвь комплексного логарифма: определим $h_{a, b}$ формулой

$$
h_{a, b}(z)=\frac{z-a}{z-b}
$$

Пусть $\log _{C}$ обозначает ветвь логарифма такую, что $\log _{C}\left(h_{a, b}(z)\right)$ имеет разрез вдоль $C$; тогда

$$
\varphi_{0}(z)=\frac{i}{4 g R}\left(\left(e-e^{*}\right) \log _{C}\left(h_{a, b}(z)\right)+\left(e+e^{*}\right) \overline{\log _{C}\left(h_{a, b}(z)\right)}\right) .
$$

Гауссов интеграл, который следует вычислить, имеет вид

$$
\int d X \exp \left(-\frac{1}{2} t X \cdot A \cdot X+{ }^{t} X \cdot J\right)
$$

Вычисляя его по методу седловой точки, получим

$$
\exp \left(\frac{1}{2}^{t} J \cdot X_{0}\right) \quad \text { c } \quad A \cdot X_{0}=J
$$

Однако, как мы увидим ниже, использование этого анзаца требует осторожности из-за возникающих расходимостей.

Вычислим первый вклад, появляющийся при вычислении в седловой точке. Мы должны перенормировать выражение

$$
\exp \left(\frac{i e}{2 R}\left(\varphi_{0}(b)-\varphi_{0}(a)\right)\right)
$$

Выбираем предписание, в котором надо заменить $\varphi_{0}(a)$ и $\varphi_{0}(b)$ их средними значениями по окружностям радиуса $\varepsilon$ с центрами соответственно в точках $a$ и $b$. Тогда мы получим регуляризованные величины, зависяшие от $\varepsilon$. Затем можно использовать схему 
минимального вычитания и забыть про расходимости. Обозначим через $\Gamma_{\varepsilon}^{(1)}\left(a, b, e, e^{*}\right)$ регуляризованную величину, определенную следующим образом:

$$
\left\langle\exp \left\{\frac{i e}{2 R}\left(\varphi_{0}(b)-\varphi_{0}(a)\right)\right\}\right\rangle_{\varepsilon}=\exp \left\{-\Gamma_{\varepsilon}^{(1)}\left(a, b, e, e^{*}\right)\right\} .
$$

В ведуших порядках мы получим

$$
\Gamma_{\varepsilon}^{(1)}\left(a, b, e, e^{*}\right) \simeq-\frac{e^{2}}{2 \alpha} \log (\varepsilon)+\frac{e^{2}}{2 \alpha} \log \left(\left|h_{a, b}(z)\right|\right)-\frac{e e^{*}}{2 \alpha} \log \left(\frac{h_{a, b}(z)}{\overline{h_{a, b}(z)}}\right) .
$$

Чтобы получить другие вклады, заметим просто, что

$$
\begin{aligned}
d \varphi & =\partial_{z} \varphi d z+\partial_{\bar{z}} \varphi d \bar{z}, \\
d^{*} \varphi & =i\left(\partial_{\bar{z}} \varphi d \bar{z}-\partial_{z} \varphi d z\right),
\end{aligned}
$$

и, таким образом, второй вклад получается из первого перестановкой $e$ и $e^{*}$. Наконец, имеем

$$
\Gamma_{\varepsilon}^{(2)}\left(a, b, e, e^{*}\right) \simeq-\frac{\left(e^{*}\right)^{2}}{2 \alpha} \log (\varepsilon)+\frac{\left(e^{*}\right)^{2}}{2 \alpha} \log \left(\left|h_{a, b}(z)\right|\right)-\frac{e e^{*}}{2 \alpha} \log \left(\frac{h_{a, b}(z)}{\overline{h_{a, b}(z)}}\right) .
$$

Учитывая оба вклада и вычитая общую расходимость $\varepsilon^{-\left(e^{2}+\left(e^{*}\right)^{2}\right) / 2 \alpha}$, найдем требуемую корреляционную функцию, т.к. статистическая сумма на сфере сокрашается в числителе и знаменателе. Окончательно получим

$$
\left\langle\exp \left\{\frac{i e}{R} \int_{C} d \varphi+\frac{e^{*}}{R} \int_{C} d^{*} \varphi\right\}\right\rangle_{\mathbb{P}_{1}(\mathbb{C})}=(b-a)^{-\left(e-e^{*}\right)^{2} / 4 \alpha}(\overline{b-a})^{-\left(e+e^{*}\right)^{2} / 4 \alpha} .
$$

Вспоминая условия квантования, выведенные в разделе 6.1 , и стандартную форму двухточечной корреляционной функции в 2D-KTП [20], мы восстановим спектр свободного компактифицированного бозона в модулярно-инвариантном секторе: $e^{*}=2 \alpha n$ и $e=$ $-m$. В дальнейшем $\phi_{n, m}(z, \bar{z})$ будет обозначать $\widehat{U(1)}$-примарное поле с импульсами $\left(p_{n, m}, \bar{p}_{n, m}\right)$. Уравнение (6.14) задает коррелятор $\left\langle\phi_{n, m}^{\dagger}(b) \phi_{n, m}(a)\right\rangle$.

\section{3. Корреляционные функции на торе}

Чтобы проделать такое же вычисление на торе, требуются три шага. Идея состоит в том, чтобы расшепить бозонное поле на несколько частей:

1) обычный инстантонный вклад, зависящий от чисел намоток вдоль двух гомологически независимых циклов тора;

2) вклад из интеграла в седловой точке с нулевой монодромией вдоль гомологических циклов тора, но с учетом вставок вершинных операторов;

3) вклад осцилляций.

Проблема перенормировки возникает только при вычислении вклада осцилляций. Для того чтобы вычислить статистические суммы, мы используем формулу пересуммирования Пуассона. При этом мы приводим лишь конечный результат. Детали можно найти в приложении D. 
Введем функции

и

$$
Z(a, b)=\omega_{1} \frac{\vartheta_{1}\left(\frac{b-a}{\omega_{1}}, \tau\right)}{\vartheta_{1}^{\prime}(0, \tau)}
$$

$$
\mathcal{F}_{\left[\left(e_{k}\right)_{k}\right]}\left(z_{1}, \ldots, z_{n}\right)=\prod_{k \neq l} Z\left(z_{k}, z_{l}\right)^{e_{k} e_{l} / 8 \alpha} .
$$

Чтобы задать вклад тета-функции, нам понадобятся следующие дивизоры [79] на торе $\left(e_{k}^{ \pm}=e_{k} \pm e_{k}^{*}\right)$ :

$$
D_{-}=\sum_{k=1}^{n} e_{k}^{-\frac{z_{k}}{\omega_{1}}}, \quad \bar{D}_{+}=\sum_{k=1}^{n} e_{k}^{+} \frac{\bar{z}_{k}}{\bar{\omega}_{1}}
$$

Тогда этот вклад примет вид

$$
\Theta_{\Gamma_{L}}\left(\tau, \bar{\tau}, D_{-}, \bar{D}_{+}\right)=\sum_{(p, \bar{p}) \in \Gamma_{L}} q^{\frac{1}{2} p^{2}} \bar{q}^{\frac{1}{2} \bar{p}^{2}} \exp \left(i \frac{\pi}{\sqrt{\alpha}}\left(p D_{-}+\bar{p} \bar{D}_{+}\right)\right)
$$

а корреляционная функция будет даваться довольно сложным выражением

$$
\begin{aligned}
\left\langle\prod_{k=1}^{n} V_{\left(e_{k}, e_{k}^{*}\right)}\left(z_{k} \cdot \bar{z}_{k}\right)\right\rangle_{\mathbf{T}_{\tau}}= & \frac{\Theta_{\Gamma_{L}}\left(\tau, \bar{\tau}, D_{-}, \bar{D}_{+}\right)}{\Theta_{\Gamma_{L}}(\tau, \bar{\tau}, 0,0)} \times \\
& \times \mathcal{F}_{\left[\left(e_{k}^{-}\right)_{k}\right]}\left(z_{1}, \ldots, z_{n}\right) \overline{\mathcal{F}_{\left[\left(e_{k}^{+}\right)_{k}\right]}\left(z_{1}, \ldots, z_{n}\right)} .
\end{aligned}
$$

Оно учитывает все эффекты конечного размера в корреляционных функциях. Определим асимптотики этой корреляционной функции в пределе совпадающих точек, т.е. когда

$$
\forall k \neq l, \quad\left|z_{k}-z_{l}\right| \ll \min \left(\left|\omega_{1}\right|,\left|\omega_{2}\right|\right) .
$$

В этом пределе $D_{-}$и $\bar{D}_{+}$обращаются в нуль, а $Z(a, b) \simeq b-a$. Таким образом, мы воспроизводим выражение, полученное выше для корреляционных функций на римановой сфиере.

Отметим, что эти корреляционные функции обладают обычной для КТП структурой [51] билинейной комбинации конформных блоков:

$$
\sum_{I, \bar{I}} \mathcal{F}_{I}(x) \overline{\mathcal{F}_{\bar{I}}}(\bar{x})
$$

где $x$ обозначает некоторую комплексную координату на соответствуюшем пространстве Тейхмюллера. Здесь это пространство - пространство Тейхмюллера комплексного тора с $n+1$ отмеченной точкой ${ }^{6)}$ и с подходящей спиновой структурой на эллиптической кривой. Полученное выражение подразумевает, что эта корреляционная функция была вычислена с использованием специальной области пространства Тейхмюллера: все точки $z_{k}$ и $z_{0}$ должны лежать в элементарной ячейке решетки периодов тора. $\widehat{U(1)}$-конформные блоки имеют вид

$$
\mathcal{F}_{\left[\left(e_{k}\right)_{k}, p\right]}=\frac{q^{\frac{1}{2} p^{2}}}{\eta(\tau)} \exp \left\{i \frac{\pi}{\sqrt{\alpha}} p D_{-}\right\} \mathcal{F}_{\left[\left(e_{k}^{-}\right)_{k}\right]}\left(z_{1}, \ldots, z_{n}\right),
$$

\footnotetext{
${ }^{6)}(n+1)$-я точка является точкой начала отсчета $P_{0}$ и не входит в выражение явно.
} 
где $p$ равно $p_{l / 2, m} \mathrm{c} l \equiv m(\bmod 2)$.

В восьмидесятые годы похожие формулы возникали в рамках струнной теории и в КТП [27]. Однако в основном интерес вызывал модулярно-инвариантный сектор статистической суммы. Более точно, выражение для корреляторов было таким же, за исключением суммы по всем импульсам $(p, \bar{p})$. В модулярно-инвариантном секторе необходимо использовать решетку $\left\{\left(p_{n, m}, \bar{p}_{n, m}\right)\right.$, где $\left.(n, m) \in \mathbb{Z}^{2}\right\}$, которая отвечает импульсам, дающим вклад в $Z_{0,0}$. В случае ЖЛ эти импульсы лежат на решетке

$$
\Gamma_{\text {Lutt }}=\left\{\left(p_{l / 2, m}, \bar{p}_{l / 2, m}\right), \text { где }(l, m) \in \mathbb{Z}^{2}, \quad l \equiv m(\bmod 2)\right\} .
$$

\section{4. Свободные фермионы}

Интересно рассмотреть эти выражения в случае свободных фермионов $(\alpha=1)$ и вычислить корреляционные функции фермионных операторов. Правые фермионы имеют конформный спин $1 / 2$. Они являются $\widehat{U(1)}$-примарными полями, т.е. киральными вершинными операторами при специальном выборе $\left(e, e^{*}\right)$. Чтобы получить конформный спин $1 / 2$, надо выбрать $e=1$ и $e^{*}=-\alpha(n=-1 / 2$ и $m=1)$. Вычислим теперь двухточечную функцию на торе:

$$
G_{\alpha=1}(a, b)=\left\langle\exp \left(\frac{i}{R} \int_{C_{a b}} d \varphi-\frac{\alpha}{R} \int_{C_{a b}} d^{*} \varphi\right)\right\rangle_{\omega_{1} \mathbb{Z} \oplus \omega_{2} \mathbb{Z}} .
$$

Возьмем $\alpha=1$. В этом случае $e_{-}=2$ и $e_{+}=0$. Вклад осцилляций содержит только голоморфную часть:

$$
\omega_{1}^{-1} \frac{\vartheta_{1}^{\prime}(0, \tau)}{\vartheta_{1}(z, \tau)}
$$

где $z=(b-a) / \omega_{1}$. Вклад тета-функции дается отношением двух тета-функций. При $\alpha=1$ выражение (6.18) переходит в следующее:

$$
\sum_{(p, \bar{p}) \in \Gamma_{L}} q^{\frac{1}{2} p^{2}} \bar{q}^{\frac{1}{2} \bar{p}^{2}} e^{2 i \pi p z} .
$$

В свободном случае решетка $\Gamma_{\text {Lutt }}$ совпадает с $\mathbb{Z}^{2}$ и тогда эта тета-функция разлагается в произведение голоморфной и антиголоморфной частей.

Собирая все члены вместе, мы получим

$$
G_{\alpha=1}(a, b)=\omega_{1}^{-1} \frac{\vartheta_{1}^{\prime}(0, \tau)}{\vartheta_{1}(z, \tau)} \frac{\vartheta_{3}(z, \tau)}{\vartheta_{3}(0, \tau)},
$$

что может быть записано как

$$
G_{\alpha=1}(a, b)=\frac{\pi}{\omega_{1}} N(\tau) H(z, \tau),
$$

где $\left(y=e^{2 \pi i z}\right)$

$$
\begin{aligned}
& N(\tau)=\prod_{n=1}^{+\infty}\left(\frac{1-q^{n}}{1+q^{n-1 / 2}}\right)^{2} \\
& H(z, \tau)=\frac{1}{\sin (\pi z)} \prod_{n=1}^{+\infty} \frac{\left(1+y q^{n-1 / 2}\right)\left(1+y^{-1} q^{n-1 / 2}\right)}{\left(1-y q^{n}\right)\left(1-y^{-1} q^{n}\right)} .
\end{aligned}
$$


Это не что иное, как двухточечньй коррелятор свободных дираковских фермионов на торе, что станет понятным, если мы покажем, что выражение (6.24) удовлетворяет уравнениям Швингера-Дайсона теории Дирака. Прямое фермионное вычисление также дает тот же результат.

Разумеется, при $\alpha \neq 1$ эти корреляционные функции перемешивают голоморфную и антиголоморфную части. Более того, рассмотренные вершинные операторы $(n= \pm 1 / 2$, $m= \pm 1$ ) суть перенормированные фермионы в теории со взаимодействием. Более точно, если $\psi_{0, R}^{\dagger}$ рождает голый правый фермион, мы получим $\phi_{1 / 2,1}=Z^{-1 / 2} \psi_{0, R}^{\dagger}$, где $Z$ - мультипликативная константа перенормировки, которая равна нулю для локальных взаимодействий в “сценарии” катастрофы "по ортогональности” [9]. Физический смысл прозрачен: $\phi_{1 / 2,1}$ рождает нормированное состояние, в то время как $Z$ - квадрат нормы состояния, получающегося при добавлении к системе голого электрона. Тогда $Z=0$ просто означает, что добавление голого электрона к системе переводит последнюю в состояние, ортогональное всем собственным состояниям взаимодействуюшего гамильтониана. Другими словами, собственные состояния взаимодействующего гамильтониана не связаны с исходными фермионами. Константа перенормировки $Z$ также связана с разрывностью функции распределения импульса в точке $k_{\mathrm{F}}$ по теореме Мигдала (см. [4, c. 42 и 63]). Таким образом, обрашение $Z$ в нуль означает отсутствие такого разрыва в функции распределения импульса электрона на поверхности Ферми.

\section{5. Учет модельного беспорядка}

Из результатов раздела 4 следует, что мы можем явно определить функцию влияния модельного беспорядка на корреляторы вершинных операторов. Рассмотрев произведение вершинных операторов (6.3), получим

$$
\mathcal{O}\left[\varphi+\frac{\eta}{g R}\right]=\mathcal{O}[\varphi] \exp \left(\sum_{k=1}^{n} \frac{i e_{k}}{\alpha}\left(\eta\left(\sigma_{k}\right)-\eta\left(\sigma_{0}\right)\right)+\frac{e_{k}^{*}}{\alpha} \int_{C_{0 k}} d^{*} \eta\right)
$$

Разумеется, $d \eta=\eta^{\prime}(\sigma) d \sigma$ и $d^{*} \eta=\eta^{\prime}(\sigma) d t$. Функциональный интеграл

$$
\begin{aligned}
F_{1}\left(\sigma_{1}, \ldots, \sigma_{n}\right)= & \int \mathcal{D}[\eta(\sigma)] \delta(\eta(0)) \exp \left\{-\frac{1}{2 \gamma} \int_{0}^{L}\left(\eta^{\prime}(\sigma)\right)^{2} d \sigma\right\} \times \\
& \times \exp \left\{i \sum_{k} \frac{e_{k}}{\alpha}\left(\eta\left(\sigma_{k}\right)-\eta\left(\sigma_{0}\right)\right)\right\}
\end{aligned}
$$

легко вычисляется методом седловой точки. Результат выражается через $\sigma_{k l}$, которые являются представителями разности $\sigma_{k}-\sigma_{l} \in \mathbb{R} / L \mathbb{Z}$, лежащими в $[0, L[$ :

$$
F_{1}\left(\sigma_{1}, \ldots, \sigma_{n}\right)=\exp \left(-\frac{\gamma}{4 \alpha^{2}} \sum_{\substack{k \neq l \\(k, l) \in\langle 1, n\rangle^{2}}} e_{k} e_{l} \frac{\sigma_{k l}\left(L-\sigma_{k l}\right)}{L}\right)
$$


Второй вклад имеет вид

$$
\begin{aligned}
F_{2}\left(z_{1}, \ldots, z_{n}\right)= & \int \mathcal{D}[\eta(\sigma)] \delta(\eta(0)) \exp \left\{-\frac{1}{2 \gamma} \int_{0}^{L}\left(\eta^{\prime}(\sigma)\right)^{2} d \sigma\right\} \times \\
& \times \exp \left\{\sum_{k} \frac{e_{k}^{*}}{\alpha} \int_{C_{0 k}} \eta^{\prime}(\sigma) d t\right\}
\end{aligned}
$$

Для простоты вычислим только двухточечную функцию (кривая $C_{a b}$ в наших вычислениях - это линия, связывающая $z_{1}$ и $z_{2}$ ). Используя формулу

$$
\int_{C_{12}} d^{*} \eta=\frac{\Im\left(z_{12}\right)}{\Re\left(z_{12}\right)}\left(\eta\left(\Re\left(z_{2}\right)\right)-\eta\left(\Re\left(z_{2}\right)\right)\right),
$$

получим

$$
F_{2}\left(z_{1}, z_{2}\right)=\exp \left(\frac{\gamma\left(e^{*}\right)^{2}}{2 \alpha^{2}} \frac{u_{12}^{2}\left(L-\sigma_{12}\right)}{L u_{12}}\right) .
$$

Эти результаты требуется пояснить. Во-первых, экспоненциальное убывание корреляций в пространственном направлении объясняется как конечньй эффект модельного беспорядка. Подчеркнем, что экспоненциальные показатели алгебраических распадов не модифицируются. Восстанавливая конечный волновой вектор $k_{\mathrm{F}}$, мы могли бы показать, что это экспоненциальное убывание задается в конечном итоге вкладом $2 k_{\mathrm{F}}$ и не является эффектом, связанным с локализацией.

\section{7. ЭКСПЕРИМЕНТЫ ЛАВЛИНА И ЖЛ}

В этом разделе мы обсудим соотношение между некоторыми несжимаемыми жидкостями Холла и ЖЛ. Главная идея состоит в том, что в определенном пределе жидкость Холла в геометрии кольца эквивалентна ЖЛ при подходяше выбранных параметрах. В частности, мы обсуждаем поведение ДКЭХ-жидкостей в рамках КТП Люттингера.

Начнем с напоминания, как вычислять плотности физического заряда и тока в ЖЛ исходя из сохраняющихся $U(1)$-токов модельной КТП. Затем, рассмотрев мысленный эксперимент Лавлина [50] под другим углом зрения, мы соотнесем заполняющую фракцию квантового эффекта Холла $\nu=1 /(2 p+1)$ с параметром взаимодействия ЖЛ. Далее найдем соответствие между квантовыми жидкостями Холла и ЖЛ. В последующих разделах мы обсуждаем рациональные ЖЛ. Некоторые из соответствующих РКТП описывают лавлиновские состояния Холла (для заполняющей фракции $\nu=1 /(2 p+1)$ ). Чтобы задать такие РКТП, мы введем дробный заряд на крае для описания башни возбужденных состояний над заданным возмушением в толще жидкости и точно решим полученные твистованные теории.

Наконец, отметим, что подход КТП к краевым возмушениям широко обсуждался в литературе (основные ссылки - оригинальная работа Вена [45] и его обзор [46] краевых состояний.) Здесь мы даем более простой вывод этих результатов, который обеспечивает ясную связь с результатами Лавлина и Капелли с соавторами, имеюшими отношение к описанию краевых состояний в дробном квантовом эффекте Холла [49] и его статистической сумме [52]. Рассматривая эффективную КТП в конечном объеме, мы получим ясную и обшую физическую картину краевых возмущений. 


\section{1. Плотности физических заряда и тока в ЖЛЛ}

Как показано в предыдущих разделах, ЖЛ допускает инфракрасное описание через эффективную КТП. Эта эффективная теория является теорией свободного безмассового компактифицированного бозона со специальными граничными условиями, которые учитывают граничные условия для базисных голых фермионных полей. Будучи свободной бозонной КТП, эта теория обладает $\widehat{U(1)} \times \widehat{U(1)}$-симметрией, порождаемой током с единичным спином.

В пункте 2.1.2 было показано, что физические плотности заряда и тока непосредственно связаны с $U(1)$-токами в КТП Люттингера. Восстанавливая множители скорости и заряда в $(2.26)$ и $(2.27)$, мы получим

$$
\begin{aligned}
& \rho=\frac{e}{\sqrt{\alpha}}(J(\sigma, t)+\bar{J}(\sigma, t)), \\
& j=\frac{v_{S} e}{\sqrt{\alpha}}(J(\sigma, t)-\bar{J}(\sigma, t)),
\end{aligned}
$$

где $e$ - единичный заряд. Эти плотности могут быть выражены в терминах лорановских

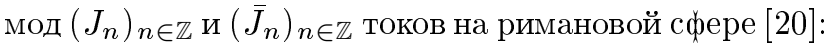

$$
\begin{aligned}
& \rho(\sigma, t)=\frac{e}{L \sqrt{\alpha}} \sum_{n \in \mathbb{Z}}\left(J_{n} \exp \left\{2 \pi i \frac{n}{L}\left(\sigma-v_{S} t\right)\right\}+\bar{J}_{n} \exp \left\{-2 \pi i \frac{n}{L}\left(\sigma+v_{S} t\right)\right\}\right), \\
& j(\sigma, t)=\frac{e v_{S}}{L \sqrt{\alpha}} \sum_{n \in \mathbb{Z}}\left(J_{n} \exp \left\{2 \pi i \frac{n}{L}\left(\sigma-v_{S} t\right)\right\}-\bar{J}_{n} \exp \left\{-2 \pi i \frac{n}{L}\left(\sigma+v_{S} t\right)\right\}\right) .
\end{aligned}
$$

Таким образом, полный заряд и средний электрический ток вдоль кольца задаются в терминах мод разложения Фурье для токов модельной КТП следующим образом:

$$
\begin{aligned}
q & =\frac{e}{\sqrt{\alpha}}\left(J_{0}+\bar{J}_{0}\right), \\
I & =\frac{e v_{S}}{L \sqrt{\alpha}}\left(J_{0}-\bar{J}_{0}\right) .
\end{aligned}
$$

Теперь удобно ввести плотности тока по отдельности для каждой ветви $\rho_{R}$ и $\rho_{L}$, так что

$$
\rho=\rho_{L}+\rho_{R}, \quad j=v_{S}\left(\rho_{R}-\rho_{L}\right)+2 e \frac{v_{S}}{L \alpha} \chi .
$$

Второе соотношение учитывает влияние вектор-потенциала на фермионные импульсы и, следовательно, на электрический ток. Приведем обычное нерелятивистское выражение для тока:

$$
J_{\mu}=\frac{e \hbar}{2 m i}\left(\psi \partial_{\mu} \psi^{*}-\psi^{*} \partial_{\mu} \psi\right)-\frac{e^{2}}{2 m}|\psi|^{2} A_{\mu} .
$$

Здесь второй член, пропорциональньй векторному потенциалу, появляется в результате калибровочной инвариантности тока. Формулы (7.7) могут быть угаданы из свойств газа свободных фермионов в размерности два в цилиндрическом пространстве. Киральные заряды выражаются через операторы КТП:

$$
q_{R}=\frac{e}{\sqrt{\alpha}} J_{0}-e \frac{\chi}{\alpha}, \quad q_{L}=\frac{e}{\sqrt{\alpha}} \bar{J}_{0}+e \frac{\chi}{\alpha} .
$$

Эти выражения будут использованы при выводе соотношений между физикой ЖЛ и физикой квантового эффекта Холла (КЭХ). 


\section{2. Перетекание заряда между различными киральностями в ЖЛ}

Лавлин [50] и Гальперин [48] рассмотрели квантовую жидкость Холла, локализованную на кольцо или на цилиндр, пронизанньй нитью магнитного потока $\Phi$. На самом деле, хотя геометрия цилиндра не может быть реализована в полной мере в эксперименте, для нашего описания удобно рассмотреть КЭХ именно на цилиндре. Авторы работ $[50,48]$ исследовали перенос заряда между двумя краями при адиабатическом включении такого потока. В целочисленном $\mathrm{K} Э \mathrm{X}$ при увеличении потока на один квант потока $\Phi_{0}$ система возврашается в исходное состояние за исключением того, что некоторые электроны переходят с одного края на другой.

В ДКЭХ в такой ситуации переносится дробньй заряд [80], и мы должны сместить магнитный поток на несколько квантов потока, прежде чем система вернется в состояние с целым числом премешенных электронов. Более точно, для заполняюшей фракции $\nu$ перенос заряда при увеличении поля $\Phi \mapsto \Phi+\Phi_{0}$ равен $\nu e$. При $\nu=q / p$ после скачка $\Phi \mapsto \Phi+p \phi_{0}$ перемешаются $q$ электронов. Это может быть понято как воздействие электрического поля, индуцированного изменением потока в присутствии проводимости Холла $\sigma_{\mathrm{H}}=\nu e^{2} / h$.

Рассмотрим теперь ЖЛ с параметром взаимодействия $\alpha$. Статистическая сумма с магнитным потоком $\chi \Phi_{0}$ равна

$$
Z_{\mathrm{Lutt}}(\chi)=\frac{1}{|\eta(\tau)|^{2}} \sum_{\substack{(n, m) \in(\mathbb{Z} / 2) \times \mathbb{Z} \\ 2 n \equiv m(\bmod 2)}} q^{\frac{1}{2} p_{n, m+2 \chi}^{2}} \bar{q}^{\frac{1}{2} \bar{p}_{n, m+2 \chi}^{2}},
$$

где

$$
p_{n, m}=n \sqrt{\alpha}+\frac{m}{2 \sqrt{\alpha}}, \quad \bar{p}_{n, m}=n \sqrt{\alpha}-\frac{m}{2 \sqrt{\alpha}} .
$$

Интересными состояниями будут $\widehat{U(1)} \times \widehat{U(1)}$-состояния старшего веса. Введем следующие обозначения:

$$
|n, m\rangle_{\chi}=\left|p_{n, m+2 \chi}, \bar{p}_{n, m+2 \chi}\right\rangle .
$$

Вектор $|n, m\rangle_{\chi}$ можно рассматривать как состояние $\left|p_{n, m}, \bar{p}_{n, m}\right\rangle$, непрерывно деф̆ормируемое внешним магнитным потоком.

Статистическая сумма (7.10) дает спектр зарядов на обоих краях. Для состояния $|n, m\rangle_{\chi}$ мы получим

$$
\begin{aligned}
& q_{R}=e\left(n+\frac{m}{2 \alpha}\right), \\
& q_{L}=e\left(n-\frac{m}{2 \alpha}\right) .
\end{aligned}
$$

Полный заряд, таким образом, будет 2 ne, т.е., почти целое число. Тем не менее, заряды на каждом крае могут быть дробными. Заметим, что плотности флуктуаций заряда, которые соответствуют $\widehat{U(1)}$-потомкам, глобально нейтральны: они не меняют левых и правых полных зарядов.

Исследуем более подробно перенос заряда между киральностями в КТП Люттингера. Это позволит нам угадать соответствие между краевыми возмушениями ДКЭХ-жидкости при $\nu=1 / q$ и подходяшей КТП Люттингера. 


\section{3. Эксперимент Лавлина}

Начнем с КТП Люттингера, имеюшей параметр взаимодействия $\alpha$. При нулевых температуре и магнитном потоке система находится в состоянии $\left|p_{0,0}, \bar{p}_{0,0}\right\rangle=|0,0\rangle_{\chi=0}$. Киральные заряды $q_{R}$ и $q_{L}$ в этом состоянии равны нулю. Если мы теперь будем адиабатически увеличивать магнитный поток от значения $\chi=0$ к конечной величине $\chi \in\left[0,1 / 2\left[\right.\right.$, система адиабатически перейдет в состояние $|0,0\rangle_{\chi}$. В этом промежуточном состоянии по-прежнему $q_{L}=q_{R}=0$, но возникает ненулевой ток

$$
I=\frac{2 e v_{S}}{L \alpha} \chi
$$

При достижении $\chi$ величины $1 / 2$ (именно, при $1 / 2<\chi<3 / 2$ ) наинизшим энергетическим состоянием оказывается вместо $|0,0\rangle_{\chi}$ состояние $|0,-2\rangle_{\chi}$. В этом новом состоянии заряд переносится с одной киральности на другую:

$$
\begin{aligned}
q_{R} & =\frac{e}{\alpha}, \\
q_{L} & =-\frac{e}{\alpha} .
\end{aligned}
$$

Сразу видно, что это поведение похоже на поведение ДКЭХ-жидкости, описанное Лавлином [50] и Тао и Ву [80]. Если теперь отождествить левые и правые ветви ЖЛ с двумя различными краями цилиндрического образца, заряд, переносимый с одного края на другой, будет равен $e / \alpha$, как и получил Лавлин. Таким образом, заполняюшая фракция $\nu$ должна относиться к параметру взаимодействия как $\alpha \nu=1$. Предполагая выполнение этого соотношения, можно непосредственно вычислить проводимость Холла с помошью КТП Люттингера: предположим, что два края образца не связаны с какими-либо источниками заряда, и будем увеличивать магнитный поток $\chi$. При увеличении потока система проходит через различные состояния:

$$
|0,0\rangle_{\chi} \longrightarrow|0,-2\rangle_{\chi} \longrightarrow|0,-4\rangle_{\chi} \longrightarrow \cdots
$$

Заряд края, таким образом, представляет собой ступенчатую функцию магнитного потока. Более точно, для $k+1 / 2<\chi<k+3 / 2$ мы получим $q_{R}=-q_{L}=e \nu k$. Когда $k$ достигнет величины $p-1 / 2$, получим $q_{R}=-q_{L}=e \nu p=q e$, что и следует из выводов Тао и Ву. Как мы увидим в разделе 9.3 , состояние $|0,-2 q\rangle_{\chi}$ получается при уничтожении электронов на левом крае и появлении их на правом крае. Если магнитный поток линейно возрастает по времени, индукция порождает орторадиальное электрическое поле. Тогда-то заряды и переносятся с одного края на другой. Напряжение между краями задается формулой

и скорость переноса зарядов -

$$
U=\frac{h}{e} \frac{d \chi(t)}{d t}
$$

$$
I_{\perp}=e \nu \frac{d \chi(t)}{d t} .
$$

Таким образом, мы получим

$$
I_{\perp}=\frac{e^{2}}{h} \nu U
$$

т.е. правильную формулу для проводимости Холла. Как мы уже видели, продольная интенсивность есть периодическая функция потока с нулевым средним. Таким образом, продольная проводимость равна нулю. 


\section{4. Другой эксперимент по переносу заряда}

В другом эксперименте по переносу заряда на двух краях образца поддерживаются различные потенциалы. В этом случае вдоль кольца Холла возникает ток. Покажем, как это можно получить из эффективной КТП Люттингера, чтобы еше раз подтвердить соответствие между эффективной теорией краевых возмушений и подходяшей КТП Люттингера.

Подсоединим два края цилиндра к различным электрическим потенциалам:

$$
H_{\text {elec }}=-\int_{0}^{L}\left(V_{R}(\sigma) \rho_{R}(\sigma)+V_{L}(\sigma) \rho_{L}(\sigma)\right) d \sigma .
$$

Предполагая, что $\chi=0$, мы можем записать

$$
H_{\text {elec }}=-\int_{0}^{L} V(\sigma) \rho(\sigma) d \sigma-\int_{0}^{L} \frac{V_{R}(\sigma)-V_{L}(\sigma)}{2 v_{S}} j(\sigma) d \sigma,
$$

где $V(\sigma)=\left(V_{R}(\sigma)+V_{L}(\sigma)\right) / 2$ и $U_{\perp}=V_{R}-V_{L}$. Таким образом, электрический потенциал между двумя краями может быть представлен как векторный потенциал

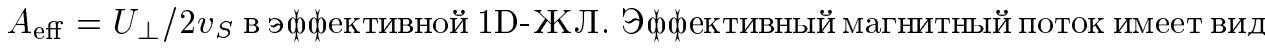

$$
\chi_{\mathrm{eff}}=\frac{e L}{2 h v_{S}} U_{\perp} .
$$

Можно попробовать применить наши предыдушие рассуждения, касаюшиеся электрического тока, индуцированного магнитным потоком в ЖЛ. Однако, как было вычислено в разделе 5.3, ток в цилиндре среднего размера должен быть периодичен по магнитному потоку. Такое поведение не ожидается в эксперименте Холла. Разрешение этого очевидного парадокса состоит в том, что в эксперименте Холла края соединены с электрическим генератором, который, кроме того что накладывает напряжение $U_{\perp}$, может привносить заряды в систему. Обсудим это более подробно в предположении, что $\alpha=q \in 2 \mathbb{N}+3$.

Когда поток достигает значения $1 / 2$, на краю возникает заряд $\pm e / q$. Внешний генератор не привносит никакого заряда, чтобы нейтрализовать его, т.к. внешний генератор может привносить только целые заряды. Следовательно, пока что ничего, что отличалось бы от случая изолированного кольца Люттингера, не происходит. Периодичность по току разрушается, когда полный заряд становится равен $\pm e$ на каждом крае. Тогда генератор уже может нейтрализовать заряд на каждом крае. При $\chi$, немного меньших чем $q-1 / 2$, система находится в состоянии

$$
|0,2(1-q)\rangle_{\chi}
$$

Когда $\chi$ становится больше чем $q-1 / 2$, вместо возврашения в состояние

$$
\left|p_{0,0}, \bar{p}_{0,0}\right\rangle=|0,-2 q\rangle_{\chi}
$$

с левыми и правыми зарядами $q_{L}=-q_{R}=1$ система скачком перемещается в состояние $|0,0\rangle_{\chi}$. В этом состоянии мы опять получим $q_{L, R}=0$, но ток уже не будет нулевым. 
Заметим, что в результате краевых эффектов энергия изменяется. Изменение энергии вносится внешним электрическим генератором. Электрическая проводимость Холла может быть легко вычислена. В терминах эффективного магнитного потока "классический” ток Холла дается формулой

$$
I=\frac{2 v_{S} e}{L \alpha} \chi_{\text {eff }}
$$

Таким образом, вводя разность потенциалов между двумя краями системы, мы получим

$$
I=\frac{e^{2}}{h} \alpha^{-1} U_{\perp}=\frac{e^{2}}{h} \nu U_{\perp} .
$$

Разумеется, полученный результат сильно зависит от механизма переноса заряда между электрическим генератором и образцом Холла. Правильный подход в этом случае должен использовать явное описание присоединения двух краев к источникам заряда при различных химических потенциалах и квантовые описания этих двух источников. Напомним, что туннелирование между киральностями сквозь барьер Холла широко исследовалось в последние годы (см. [81] и [82, раздел V.A]). Для амплитуд туннелирования, свойств переноса и неравновесного шума постоянного тока непертурбативные результаты были получены с помощью методов анзаца Бете [83-86]. Затем был развит формфакторньй подход к этим проблемам [87]. Расширение этих вычислений на случай конечных размеров требует знания формфакторов систем конечных размеров. Для этого необходимо исследовать КТП с центральным зарядом $c<1$, отметим совсем недавнюю статью [88], где разделение переменных по методу Склянина было применено для получения квазиклассических оценок формфакторов систем с конечным размером. Очевидно, что требуется немало усилий для описания эффектов конечного размера и свойств модели в некритическом режиме, соответствуюшем эффективному описанию дробной квантовой жидкости Холла с примесями. С экспериментальной стороны отметим работу [89], в которой описаны эксперименты по туннелированию в случае $\nu=1 / 3$. Этот важный и трудный эксперимент, который основан на измерении токового шума, обусловленного туннелированием заряда между двумя краями, кажется, свидетельствует в пользу существования дробно заряженных краевых возмущений.

Подводя итог, отметим, что свойства переноса в квантовой жидкости Холла могут быть объяснены в рамках КТП Люттингера. Важно, что не все ЖЛ получаются из квантовых жидкостей Холла: параметр взаимодействия $\alpha$ должен быть рациональным числом. Естественно, наш подходнегодится для описания переходов между плоскостями Холла. Мы можем лишь описать эффективную теорию краевых возмушений на данной плоскости.

Интересно понять более точно, почему эти рациональные значения столь важны. Это основная причина исследования КТП Люттингера с точки зрения РКТП.

\section{8. РАЦИОНАЛЬНЫЕ КТП ЛЮТТИНГЕРА}

РКТП возникли впервые в работе Фредана и Шенкера [51]. Грубо говоря, это КТП с очень высокой алгеброй симметрий - столь высокой, что гильбертово пространство состояний будет прямой суммой конечного числа неприводимых представлений этой алгебры симметрий (см. обзор [90]). По аналогии ЖЛ называется рациональной тогда и только тогда, когда эффективная теория является РКТП. 
Начнем с вывода условия рациональности для ЖЛ. Будут вычислены обобщенные характеры расширенной алгебры симметрий. Это позволит нам выразить статистическую сумму в терминах расширенных характеров. Как мы увидим, статистические суммы, полученные недавно Капелли и сотрудниками [52], в этот список включены.

\section{1. Критерий рациональности для КТП Люттингера}

Статистическая сумма КТП Люттингера дается уравнениями (7.10) и (7.11). Если эффективная теория является РКТП, то должны сушествовать конформные примарные поля размерностей $(h, 0)$, расширяющие алгебру $\widehat{U(1)}$. Это подразумевает сушествование условия, сходного с ограничением для свободной модулярно-инвариантной бозонной теории: $\alpha / 2 \in \mathbb{Q}$. Здесь же мы обнаружим, что $\alpha$ - рациональное число. Пусть $\alpha=q / p$, где $q$ и $p$ суть взаимно простые положительные целые числа.

Конформные размерности $(h, \bar{h})$ полей $\phi_{l / 2, m}$ даются формулой

$$
\left(\frac{(l q+m p)^{2}}{8 p q}, \frac{(l q-m p)^{2}}{8 p q}\right) .
$$

Здесь $l$ и $m$ - целые числа одинаковой четности (условие конгруэнтности). Из леммы Гаусса [91] мы получим, что если $\phi_{l / 2, m}$ имеет нулевую размерность $\bar{h}$, то сушествует $k \in \mathbb{Z}$ такое, что $l=k p$ и $m=k q$. В этом случае мы получим

$$
h_{k p / 2, k q}=\frac{p q}{2} k^{2} .
$$

Тем не менее в модели Люттингера выполнено условие конгруэнтности $l \equiv m(\bmod 2)$, и, таким образом, следующие два случая будут обсуждаться по отдельности.

8.1.1. Случай, когда $p \equiv q(\bmod 2)$. Так как $p$ и $q$ взаимно простые, оба числа нечетные. Условие конгруэнтности выполнено при всех $k \in \mathbb{Z}$. Голоморфные $\widehat{U(1)}$-примарные поля являются полями $\phi_{k p / 2, k q}$ при всех $k \in \mathbb{Z}$. При четных $k$ размерность $\bar{h}-$ целое число, а при нечетных $k$ - полуцелое. Обозначим через $\mathcal{A}$ киральную алгебру, порождаемую $U(1)$-током $J(z)$ и всеми полями $\phi_{k p / 2, k u}$ для $k \in \mathbb{Z}$. Эта операторная алгебра $\mathcal{A}$ обладает $\mathbb{Z}_{2}$-градуировкой $2 h(\bmod 2)$, и мы запишем $\mathcal{A}=\mathcal{A}_{+} \oplus \mathcal{A}_{-}$. Поля $\phi_{k p / 2, k u}$ для $k \in 2 \mathbb{Z}+1$ лежат в алгебре $\mathcal{A}_{-}$, а поля $\phi_{k p, 2 k u}-$ в алгебре $\mathcal{A}_{+}$.

8.1.2. Случай, когда $p \equiv q+1(\bmod 2)$. Одно из этих двух целых чисел четно. Таким образом, условие конгруэнтности выполнено лишш при четном $k$. Голоморфные $\widehat{U(1)}$-примарные поля будут полями $\phi_{k p, 2 k q}$ при всех значениях $k \in \mathbb{Z}$. Киральная алгебра содержит только поля целого спина.

\section{2. Расширенные характеры}

Расширенные характеры определены в КТП следующим образом: пусть $\mathcal{V}$ - неприводимое представление киральной алгебры ${ }^{7)}$, тогда

$$
\chi \mathcal{V}(\tau)=\operatorname{Tr} \mathcal{V}\left(q^{L_{0}-c / 24}\right),
$$

\footnotetext{
7)Эта алгебра содержит алгебру Вирасоро.
} 
где $q=\exp (2 \pi i \tau)$. Как показал Карди [22], эти характеры суть конформные блоки статистической суммы КТП на торе.

Расширенная алгебра образована голоморфными полями целого спина в КТП Люттингера. Легко получить и расширенные характеры. Поля $\phi_{k p, 2 k q}$ и их правые $\widehat{U(1)}$-потомки порождают киральную алгебру $\mathcal{A}_{c}$. В терминах собственного значения $p$ оператора $J_{0}$ они соответствуют $p \in 2 \sqrt{p q} \mathbb{Z}$. Неприводимое представление $\mathcal{A}_{c}-$ это прямая сумма $\widehat{U(1)}$-неприводимых представлений, которая может быть задана набором собственных значений оператора $J_{0}$ вида $p_{0}+2 \sqrt{p q} \mathbb{Z}$. Найдем допустимые значения $p_{0}$. Для этого мы потребуем, чтобы лорановские моды всех киральных вершинных операторов, порождаюших $\mathcal{A}_{c}$, и кирального вершинного оператора, отвечаюшего $p_{0}$, удовлетворяли коммутационным соотношениям. Операторное произведение киральных вершинных операторов

$$
V_{p_{1}}(z) V_{p_{2}}(\xi)=(z-\xi)^{p_{1} p_{2}} V_{p_{1}+p_{2}}(\xi)
$$

как раз и дает нам такое условие. Лорановские моды $V_{p_{1}}$ и $V_{p_{2}}$ удовлетворяют коммутационным соотношениям тогда и только тогда, когда $p_{1} p_{2} \in \mathbb{Z}$. Таким образом, получим $p_{0} \in(2 \sqrt{p q})^{-1} \mathbb{Z}$. Расширенные характеры даются формулой

$$
\chi_{\lambda}(\tau)=\frac{1}{\eta(\tau)} \sum_{n \in \mathbb{Z}} q^{(\lambda+4 n p q)^{2} / 8 p q},
$$

где $\lambda \in \mathbb{Z} / 4 p q \mathbb{Z}$. В терминах тета-функций с характеристиками мы получим

$$
\chi_{\lambda}(\tau)=\frac{\vartheta\left[\begin{array}{c}
\lambda / 4 p q \\
0
\end{array}\right](0,4 p q \tau)}{\eta(\tau)} .
$$

При $p \equiv q(\bmod 2)$ удобно ввести расширенные характеры $\left.{ }^{8}\right)$, определенные как

$$
\varphi_{\lambda}^{( \pm)}=\chi_{\lambda} \pm \chi_{\lambda+2 p q}
$$

где $\lambda \in \mathbb{Z} / 2 p q \mathbb{Z}$. Их свойства относительно модулярных преобразований могут быть легко получены. Их подмножество $\varphi_{2 \lambda}^{(+)}$, где $\lambda \in \mathbb{Z} / p q \mathbb{Z}$, образует унитарное представление модулярной группы $\Gamma\left(S, T^{2}\right)$ [92]. Соответствуюшая $S$-матрица [22] имеет вид

$$
\varphi_{2 \lambda}^{(+)}\left(-\frac{1}{\tau}\right)=\frac{1}{\sqrt{p q}} \sum_{\lambda^{\prime} \in \mathbb{Z} / p q \mathbb{Z}} \exp \left\{-2 \pi i \frac{\lambda \lambda^{\prime}}{p q}\right\} \varphi_{2 \lambda}^{(+)}(\tau) .
$$

Мы получим также

$$
\varphi_{2 \lambda}^{(+)}(\tau+2)=\exp \left\{2 \pi i\left(\frac{\lambda^{2}}{4 p q}-\frac{1}{12}\right)\right\} \varphi_{2 \lambda}^{(+)}(\tau) .
$$

В заключение заметим, что эффективная КТП является КТП с полями $\mathbb{Z} / N \mathbb{Z}$ в смысле работы [93] с $N=4 p q$. Однако, поскольку граничные условия, используемые в модели

\footnotetext{
8) Расширенные характеры аналогичны суперсимметричным характерам суперконформной теории поля.
} 
Люттингера, не модулярно-инвариантны по отношению к полной модулярной группе $S L(2, \mathbb{Z})$, статистическая сумма КТП Люттингера не принадлежит к списку, полученному в работе [93]. Вместо того чтобы дать полную классификацию возможных статистических сумм (см. [94], где была проведена классификация), мы выразим статистическую сумму рациональной модели Люттингера в терминах этих расширенных характеров. Наконец, подчеркнем, что рациональные неунитарные КТП иногда использовались при описании краевых возмушений некоторых ДКЭХ-жидкостей типа жидкостей в состоянии Халдейна-Резаи [95], да и для других состояний (см. [96]). Тем не менее неунитарность приводит к трудностям в соотношениях между корреляторами КТП и физическими корреляторами краевых возмущений, как было показано в [97], что противоречит выводам [98]. Все это показывает, что в более сложных жидкостях Холла (например, неабелевых) КТП-описание краевых возмущений кажется не столь прозрачным.

\section{3. Статистические суммы рациональных КТП Люттингера}

Выразим статистическую сумму рациональной модели Люттингера в терминах расширенных характеров. Полученные формулы дадут нам более глубокое понимание структуры этих теорий. В зависимости от относительной четности $p$ и $q$ будут рассмотрены опять-таки два случая.

8.3.1. Случай четных $p$ и $q$. Представим статистическую сумму Люттингера в виде суммы двух членов:

$$
Z_{1}=\frac{1}{|\eta(\tau)|^{2}} \sum_{(r, s) \in \mathbb{Z}^{2}} q^{\frac{(r q+s p)^{2}}{2}} \bar{q}^{\frac{(r q-s p)^{2}}{2}}
$$

и

$$
Z_{2}=\frac{1}{|\eta(\tau)|^{2}} \sum_{(r, s) \in \mathbb{Z}^{2}} q^{\frac{(r q+s p+p q)^{2}}{2}} \bar{q}^{\frac{(r q-s p)^{2}}{2}} .
$$

Поскольку $p$ и $q$ взаимно просты, сушествуют $(u, v) \in \mathbb{Z}^{2}$ такие, что $q u-p v=1$. Эти целые числа не единственны, на самом деле, число $q u+p v$ определено только по модулю $2 p q$. Обозначим этот класс чисел по модулю $2 p q$ через $\omega \in \mathbb{Z} / 2 p q \mathbb{Z}$. Рассмотрим следующие подмножества $\mathbb{Z}^{2}$ :

$$
\begin{aligned}
C_{\lambda} & =((\{\lambda\}+2 p q \mathbb{Z}) \times(\{\bar{\lambda}\}+2 p q \mathbb{Z})), \\
R_{1} & =\left\{(r q+s p, r q-s p) ; \quad(r, s) \in \mathbb{Z}^{2}\right\}, \\
R_{2} & =\left\{(r q+s p, r q-s p+p q) ; \quad(r, s) \in \mathbb{Z}^{2}\right\},
\end{aligned}
$$

где $(\lambda, \bar{\lambda}) \in(\mathbb{Z} / 2 p q \mathbb{Z})^{2}$. Легко доказать, что $\left(C_{\lambda, \omega \lambda}\right)_{\lambda \in \mathbb{Z} / 2 p q \mathbb{Z}}-$ подрешетка $R_{1}$ и что $\left(C_{\lambda, \omega \lambda+p q}\right)_{\lambda \in \mathbb{Z} / 2 p q \mathbb{Z}}-$ подрешетка $R_{2}$. Таким образом, мы получим

$$
\begin{aligned}
& Z_{1}=\sum_{\lambda \in \mathbb{Z} / 2 p q \mathbb{Z}} \chi_{2 \lambda}(\tau) \overline{\chi_{2 \omega \lambda}(\tau)}, \\
& Z_{2}=\sum_{\lambda \in \mathbb{Z} / 2 p q \mathbb{Z}} \chi_{2 \lambda+2 p q}(\tau) \overline{\chi_{2 \omega \lambda}(\tau)} .
\end{aligned}
$$


Суммируя эти два вклада, найдем

$$
Z_{\text {Lutt }}(\tau, \bar{\tau})=\sum_{\lambda \in \mathbb{Z} / p q \mathbb{Z}} \varphi_{2 \lambda}^{(+)}(\tau) \overline{\varphi_{2 \omega \lambda}^{(+)}(\tau)},
$$

т.е. в точности статистическую сумму, полученную Капелли с сотрудниками в [52].

8.3.2. Случай нечетного $p$ или $q$. Похожие вычисления возможны и в этом случае. Опять-таки представим статистическую сумму как два слагаемых:

$$
Z_{1}=\frac{1}{|\eta(\tau)|^{2}} \sum_{(r, s) \in \mathbb{Z}^{2}} q^{\frac{(r q+s p)^{2}}{2}} \bar{q}^{\frac{(r q-s p)^{2}}{2}}
$$

И

$$
Z_{2}=\frac{1}{|\eta(\tau)|^{2}} \sum_{(r, s) \in \mathbb{Z}^{2}} q^{\frac{(2(r q+s p)+p+q)^{2}}{2}} \bar{q}^{\frac{(2(r q-s p)+q-p)^{2}}{2}} .
$$

В точности, как и в предыдушем случае, применим теорему Безу, чтобы найти такие два числа $(u, v) \in \mathbb{Z}^{2}$, что $q u-p v=1$. Эти два числа определены не однозначно: пара $(u+$ $k p, v+k q)$, где $k \in \mathbb{Z}$, обладает тем же свойством. Определив $\omega=p u+q v$, мы получим $\omega(p+q)=q-p+2 p q(u+v)$. Так как $p$ и $q$ имеют различные четности, можно выбрать $(u, v)$ таким образом, чтобы число $u+v$ было четным. При таком выборе получим, что $\omega(p+q) \equiv q-p(\bmod 4 p q)$. Однако теперь свобода в определении $u$ и $v$ ограничена, и, таким образом, $\omega \in \mathbb{Z} / 4 p q \mathbb{Z}$. Мы также получим, что $\omega^{2}=1$ на решетке $\mathbb{Z} / 4 p q \mathbb{Z}$. Окончательно находим

$$
\begin{aligned}
& Z_{1}=\sum_{\lambda \in \mathbb{Z} / 2 p q \mathbb{Z}} \chi_{2 \lambda}(\tau) \overline{\chi_{2 \omega \lambda}(\tau)}, \\
& Z_{2}=\sum_{\lambda \in \mathbb{Z} / 2 p q \mathbb{Z}} \chi_{2 \lambda+1}(\tau) \overline{\chi_{\omega(2 \lambda+1)}(\tau)},
\end{aligned}
$$

таким образом,

$$
Z_{\mathrm{Lutt}}(\tau, \bar{\tau})=\sum_{\lambda \in \mathbb{Z} / 4 p q \mathbb{Z}} \chi_{\lambda}(\tau) \overline{\chi_{\omega \lambda}(\tau)} .
$$

Итак, мы получили полевые составляющие всех рациональных КТП Люттингера.

\section{9. ВВЕДЕНИЕ КРАЕВОГО ЗАРЯДА НА ГРАНИЦЕ}

Цель этого раздела - дать КТП-описание краевых возмущений на фоне заданных возмушений в толще квантовой жидкости Холла. Начнем с объяснения мотивов нашего вычисления, которые основаны на введении дробного краевого заряда в КТП Люттингера. Затем мы вычислим статистические суммы этих “твистованных" КТП Люттингера с краевыми зарядами и выразим их в терминах обобщенных характеров в рациональном случае. После этого мы дадим физическую интерпретацию киральной алгебры теории. Следуя Фишеру и Стоуну [53], будем рассматривать фермионы, локализованные на одном крае образца. Мы обсудим также свойства дуальности КТП Люттингера. Наконец, будут рассмотрены примеры типа жидкости Ферми и лавлиновской жидкости Холла. 


\section{1. Зачем вводить краевой заряд?}

С работы Лавлина известно, что квантовая жидкость Холла для заполняюшей фракции $1 /(2 p+1)$ несжимаема [99]. Это означает, что все возмущения в слое имеют энергетическую шель: поэтому их трудно создать в отличие, например, от случая жидкости Ферми, обладающей конечной сжимаемостью [4], в которой низколежащие квазичастичные и квазидырочные возмушения могут быть легко созданы. Как объяснил Лавлин, возмушения в толше жидкости описываются и соответствуют объектам с дробным зарядом и анионной статистикой [100].

Однако некоторые низкоэнергетические возмущения в действительности имеются в квантовой жидкости Холла, это краевые возмушения. Они присутствуют на фоне всякого возмущения в толще жидкости. В предыдущих разделах мы нашли, что краевые возмушения над объемным низшим состоянием описьваются КТП Люттингера при $\alpha=\nu^{-1}$. Здесь мы собираемся описать краевые возмушения на фоне возмушенного объемного состояния.

Для квантовой жидкости Холла на цилиндре рождение объемного возмушения предполагает появление избыточного или недостаточного дробного заряда в объеме [99]. При этом по закону сохранения заряда на крае должен появиться заряд противоположного знака, т.е. также дробный заряд. Однако тогда создание краевых возмущений не должно менять заряд на крае (кроме как на целое число квантов). Это приводит к необходимости рассматривать твистованные секторы КТП Люттингера, которые фиксируются условием конгруэнтности для полного заряда:

$$
\frac{q_{R}+q_{L}}{e} \equiv r(\bmod 1) .
$$

В этом разделе мы исследуем такие твистованные секторы.

\section{2. Статистические суммы с краевым зарядом}

Вычислим теперь статистическую сумму модели Люттингера в присутствии краевого заряда, удовлетворяющего условию (9.1). Сначала мы вычислим ее для случая константы $\alpha$ в общем положении. Затем мы возьмем рациональную константу $\alpha$ и выразим статистическую сумму через обобщенные характеры.

9.2.1. Вычисление статистической суммы. Учитывая природу появления физического зарлда в модели Люттингера, мы модифицируем граничные условия бозонного поля таким образом, чтобы было можно ввести данный по модулю 1 зарядна границе. Более точно, мы требуем, чтобы

$$
\int_{(a)} d \varphi \equiv \pi R r(\bmod \pi R) .
$$

В ранее рассмотренных случаях мы полагали $r=0$. Модифицированный функциональный интеграл в этом случае будет

$$
\begin{aligned}
\int_{\mathcal{C}(r)} \mathcal{D}[\varphi] W[\varphi]= & \frac{1}{2} \sum_{\substack{\left(\varepsilon, \varepsilon^{\prime}\right) \in\{0,1 / 2\}^{2}\\
}}(-1)^{4 \varepsilon \varepsilon^{\prime} \times} \\
& \times \int_{\substack{\Delta_{\sigma} \varphi \equiv 2 \pi R(r / 2+\varepsilon)(\bmod 2 \pi R) \\
\Delta_{u} \varphi \equiv 2 \pi R \varepsilon^{\prime}(\bmod 2 \pi R)}} \mathcal{D}_{R}[\varphi] W[\varphi] .
\end{aligned}
$$


Все вычисления могут быть проделаны так же, как и в обычном случае, и мы получим в присутствии магнитного потока $\chi \Phi_{0}$ через кольцо следующий ответ:

$$
Z_{\text {Lutt }}^{(r)}(\tau, \bar{\tau}, \chi)=\frac{1}{|\eta(\tau)|^{2}} \sum_{\substack{(n, m) \in(\mathbb{Z} / 2) \times \mathbb{Z} \\ 2 n \equiv m(\bmod 2)}} q^{\frac{1}{2} p_{n+r / 2, m+2 \chi}^{2}} \bar{q}^{\frac{1}{2} \bar{p}_{n+r / 2, m+2 \chi}^{2}}
$$

Член с $(n, m)$ в этой сумме, как и ожидалось, соответствует полному заряду $(2 n+r) e$.

9.2.2. Выражение через обобщенные характеры. Интересно выразить статистические суммы при рациональном $\alpha$ и при нулевом магнитном поле, как и раньше, в терминах обобщенных характеров. Конечно, хотя вакуумная структура зависит от рассматриваемого сектора, поля киральной алгебры могут быть использованы для перехода от одного представления $\widehat{U(1)}$ к другому. Мы выбираем $\alpha=q / p$. Рассмотрим опять-таки по отдельности два случая, отвечаюших различным $(p+q)$-четностям.

Случай нечетных $р$ и $q$. Конформные размерности $\widehat{U(1)}$-примарных полей определяются формулой

$$
\frac{((r+l) q \pm(m+2 \chi))^{2}}{8 p q}
$$

где $(l, m) \in \mathbb{Z}^{2}$ имеют одинаковую четность. Интересно рассмотреть случай, когда $r=$ $a / q$ с $a \in \mathbb{Z}$. Тогда для $\chi=0$ спектр конформных размерностей становится равным

$$
\frac{(a+l q \pm m)^{2}}{8 p q}
$$

При четном $a$ величина $l q \pm m p+q \equiv 0(\bmod 2)$, и, таким образом, как и в разделе 8.3 , мы ожидаем, что статистическая сумма будет билинейной комбинацией обобшенных характеров $\varphi_{2 \lambda}^{(+)}$. Легко доказать, что в обшем случае

$$
Z_{\mathrm{Lutt}}^{\left(\frac{a}{q}\right)}(\tau, \bar{\tau})=\sum_{\lambda \in \mathbb{Z} / 2 p q \mathbb{Z}}\left(\chi_{a+2 \lambda}(\tau)+\chi_{a+2 \lambda+2 p q}(\tau)\right) \overline{\chi_{a+2 \omega \lambda}(\tau)} .
$$

Эта статистическая сумма может быть выражена в терминах обобщенных характеров:

$$
Z_{\mathrm{Lutt}}^{\left(\frac{a}{q}\right)}(\tau, \bar{\tau})=\sum_{\lambda \in \mathbb{Z} / p q \mathbb{Z}} \varphi_{a+2 \lambda}^{(+)}(\tau) \overline{\varphi_{a+2 \omega \lambda}^{(+)}(\tau)}
$$

Случай четного $p$ или $q$. Аналогичный анализ дает следующий ответ:

$$
Z_{\mathrm{Lutt}}^{\left(\frac{a}{q}\right)}(\tau, \bar{\tau})=\sum_{\lambda \in \mathbb{Z} / 4 p q \mathbb{Z}} \chi_{a+\lambda}(\tau) \overline{\chi_{a+\omega \lambda}(\tau)},
$$

который сводится к (8.17) при $a=0$. Таким образом, мы получили операторное содержание твистованных секторов рациональных моделей Люттингера, выраженное в терминах характеров максимальных киральных алгебр. Исследование этой статистической суммы показьвает, как бесщелевые возмущения появляются на фоне возмущений в толще жидкости. 


\section{3. Правила слияния в рациональных моделях Люттингера}

Обсудим правила слияния [90] в рациональных моделях Люттингера. Статистические суммы имеют вид

$$
\sum_{i} \chi_{i}(\tau) \overline{\chi_{\sigma(i)}(\tau)}
$$

где $i$ пробегает конечный набор индексов, а $\sigma$-перестановка в этом наборе. Разумеется, это разложение сделано в базисе максимальной алгебры симметрий модели $\mathcal{A} \otimes \mathcal{A}[101]$. В этом случае обобщенное примарное поле маркируется киральным индексом $i$. Мы собираемся найти правила отбора для алгебры операторных произведений [20] в КТП Люттингера.

Инвариантность при $\tau \mapsto-1 / \tau$ показывает благодаря формуле Верлинде [102], что $i \mapsto \sigma(i)$ задает автоморфизм алгебры слияния [103]. Таким образом, правила отбора операторной алгебры нашей КТП Люттингера задаются правилами слияния кирального поля, т.е. так же, как и в обычной КТП.

Случай нечетных $p$ и $q$. Здесь можно рассмотреть теорию по отношению к алгебре $\mathcal{A}_{+}$или к расширенной алгебре $\mathcal{A}=\mathcal{A}_{+} \oplus \mathcal{A}_{-}$. Для простоты рассмотрим максимальную алгебру $\mathcal{A}$.

Введем поле $\Phi_{\lambda}(a)$, отвечаюшее расширенному характеру $\varphi_{a+2 \lambda}^{(+)}$. При этом получаются следующие правила слияния:

$$
\Phi_{\lambda}(a) \Phi_{\lambda^{\prime}}\left(a^{\prime}\right)=\Phi_{\lambda+\lambda^{\prime}}\left(a+a^{\prime}\right) .
$$

В секторе $a=0$ присутствуют правила слияния с индексами, лежашими в конечном поле $\mathbb{Z} / p q \mathbb{Z}$, как отмечено Капелли с сотрудниками [52]. Однако киральная алгебра $\mathcal{A}$ содержит поля с полуцельми спинами. Выбор киральной алгебры, содержашей лишь поля целого спина, дает правила слияния для полей с индексами из числового поля $\mathbb{Z} / 4 p q \mathbb{Z}$.

Случай четных $p$ или $q$. Обозначим через $\Phi_{\lambda}(a)$ примарное поле с индексами $(a+\lambda, a+\omega \lambda)$. Оно принадлежит зарядовому сектору $a / q$. Из правил слияния для $\widehat{U(1)}$-киральной алгебры мы находим

$$
\Phi_{\lambda}(a) \Phi_{\lambda^{\prime}}\left(a^{\prime}\right)=\Phi_{\lambda+\lambda^{\prime}}\left(a+a^{\prime}\right),
$$

это отражает как сохранение граничного заряда $a / q$, так и то, что мы получили КТП с полями $\mathbb{Z} / 4 p q \mathbb{Z}$. Взяв в качестве исходного поле $\Phi_{\lambda}(a)$, другие поля $\Phi_{\lambda^{\prime}}(a)$ мы получим слияниями с $\Phi_{\mu}(0)$.

Физическая интерпретация. Поле $\Phi_{\lambda}(a)$ не только создает граничное возмущение, но и переводит модель в другой сектор КТП Люттингера. В каком-то смысле эти поля создают возмушения в толще жидкости. Все потомки состояния, рождаемого полем $\Phi_{\lambda}(a)$, удовлетворяют условию $q_{L}+q_{R} \equiv a / q(\bmod 1)$. Мы можем сдвинуть $\lambda$ на фиксированное число $a$ с помошью оператора вида $\Phi_{\mu}(0)$, которьй не меняет зарядового сектора.

Наконец, подчеркнем, что даже в твистованных секторах гильбертово пространство состояний все еше является представлением той же расширенной киральной алгебры $\mathcal{A}$, 
что и в объемном вакуумном секторе. В этом смысле эта киральная алгебра является симметрийной алгеброй неразветвленных краевых возмущений ДКЭХ-жидкости. Отсюда сразу возникает вопрос о физическом смысле полей, порождающих алгебру расширенных симметрий. Оказывается, что этот вопрос имеет отношение к проблеме фермионов, локализованных на одном крае.

В геометрии цилиндра физически можно ввести электроны на одном из краев системы (через туннельный переход, присоединенный к одному из краев системы). Поля, соответствующие рождению или уничтожению этих краевых фермионов, должны присутствовать во всех твистованных КТП Люттингера, отвечающих последовательности $\nu=1 /(2 p+1)$. Более того, они должны нести электрический заряд, локализованньй на одном из краев. Вследствие соотношения между конформными размерностями и краевым зарядом $\widehat{U(1)}$-примарных полей поля, несущие нулевой заряд на левом (соответственно, правом) крае, отвечают голоморфным (соответственно, антиголоморфным) примарным полям и, таким образом, киральной алгебре КТП Люттингера.

Напомним, что для $\alpha=q \in 2 \mathbb{N}+3$ (отвечающей жидкости Лавлина) киральная алгебра порождается двумя полями $\phi_{1 / 2, q}$ и $\phi_{1 / 2,-q}$. В табл. 1 даны параметры $(n, m)$, левые и правые заряды и конформные размерности этих полей и их эрмитово сопряженHых $^{9)}$.

Таблища 1

\begin{tabular}{|c|c|c|c|c|c|c|}
\hline Поле & $n$ & $m$ & $q_{R}$ & $q_{L}$ & $h$ & $\bar{h}$ \\
\hline \hline$\psi_{R, e}^{\dagger}$ & $1 / 2$ & $q$ & 1 & 0 & $q / 2$ & 0 \\
\hline$\psi_{R, e}$ & $-1 / 2$ & $-q$ & -1 & 0 & $q / 2$ & 0 \\
\hline$\psi_{L, e}^{\dagger}$ & $1 / 2$ & $-q$ & 0 & 1 & 0 & $q / 2$ \\
\hline$\psi_{L, e}$ & $-1 / 2$ & $q$ & 0 & -1 & 0 & $q / 2$ \\
\hline
\end{tabular}

Как ожидалось, эти поля несут единичный заряд, локализованный на одном из двух краев, что доказывает их отождествление с краевыми фермионами ДКЭХ-жидкости. Первая колонка в табл. 1 детализует действие этих полей. Они были отождествлены с краевыми фермионами ДКЭХ-жидкости Стоуном и Фишером [53]. Аргументация авторов этой статьи основывалась на проблеме установления соотношения между корреляционными функциями КТП Люттингера и волновой функцией Лавлина.

Наконец, вспомним, что $U(1)$-токи, принадлежашие киральной алгебре КТП Люттингера при всех значениях параметра $\alpha$, порождают нейтральные возмушения, которые являются несжимаемыми деформациями ДКЭХ-жидкости. Таким образом, структура краевых возмушений оказывается весьма простой: все эти возмушения получаются из конечного набора элементарных возмущений, отвечающих примарным полям $\Phi_{\lambda}(a)$ по отношению к расширенной алгебре $\mathcal{A}$ путем прибавления или вычитания фермионов, локализованных на краях, и деформации граничных условий на краях с помошью $U(1)$-токов. Фермионы Люттингера порождают все возмущения в нетвистованной $(a=0)$ КТП Люттингера. Они совпадают с краевыми фермионами только при $\alpha=1$.

\footnotetext{
9) Нижний индекс $е$ напоминает, что мы имеем дело с краевыми фермионами, которые не следует путать с фермионами Люттингера.
} 
Табл. 2 содержит соответствуюшую информацию для фермионов Люттингера.

Таблица 2

\begin{tabular}{|c|c|c|c|c|c|c|}
\hline Поле & $n$ & $m$ & $q_{R}$ & $q_{L}$ & $h$ & $\bar{h}$ \\
\hline \hline$\psi_{R, \text { Lutt }}^{\dagger}$ & $1 / 2$ & 1 & $\left(1+q^{-1}\right) / 2$ & $\left(1-q^{-1}\right) / 2$ & $\left(q^{1 / 2}+q^{-1 / 2}\right)^{2} / 8$ & $\left(q^{1 / 2}-q^{-1 / 2}\right)^{2} / 8$ \\
\hline$\psi_{R, \text { Lutt }}$ & $-1 / 2$ & -1 & $-\left(1+q^{-1}\right) / 2$ & $-\left(1-q^{-1}\right) / 2$ & $\left(q^{1 / 2}+q^{-1 / 2}\right)^{2} / 8$ & $\left(q^{1 / 2}-q^{-1 / 2}\right)^{2} / 8$ \\
\hline$\psi_{L, \text { Lutt }}^{\dagger}$ & $1 / 2$ & -1 & $\left(1-q^{-1}\right) / 2$ & $\left(1+q^{-1}\right) / 2$ & $\left(q^{1 / 2}-q^{-1 / 2}\right)^{2} / 8$ & $\left(q^{1 / 2}+q^{-1 / 2}\right)^{2} / 8$ \\
\hline$\psi_{L, \text { Lutt }}$ & $-1 / 2$ & 1 & $-\left(1-q^{-1}\right) / 2$ & $-\left(1+q^{-1}\right) / 2$ & $\left(q^{1 / 2}-q^{-1 / 2}\right)^{2} / 8$ & $\left(q^{1 / 2}+q^{-1 / 2}\right)^{2} / 8$ \\
\hline
\end{tabular}

\section{4. Дуальность в КТП Люттингера}

Свободная бозонная модулярно-инвариантная теория обладает электромагнитной дуальностью, играющей важную роль в обсуждении КТП с центральным зарядом $c=1$. Поэтому интересно узнать, имеется ли такая дуальность в КТП Люттингера. Нет ничего удивительного в том, что ответ положителен, но мы найдем некоторые отличия от обычной модулярно-инвариантной теории.

Чтобы прояснить свойства дуальности обычного свободного бозона и КТП Люттингера, взаимодействующей с калибровочным полем, мы используем функциональные методы. Эти методы предполагают выполнение преобразования Хаббарда-Стратоновича, которое вводит один-форму $b=b_{\mu} d x^{\mu}$. Интегрирование по исходному бозонному полю дает гармоники, а именно, точную и ко-точную части формы $b$, которые используются для построения дуального поля. Для сохранения целостности изложения все необходимые технические детали собраны в приложении $\mathrm{H}$.

Дуальность действует по-разному на константы связи обычной бозонной теории и теории Люттингера. В обычной модулярно-инвариантной бозонной теории $\alpha$ переходит в $1 / 4 \alpha$, в то время как в КТП Люттингера $\alpha$ становится равной $1 / \alpha$. Теория Дирака теперь отвечает фиксированным точками преобразований дуальности, действуюших на КТП Люттингера, а не теории в точке $\alpha=1 / 2$, отвечающей модулярно-инвариантному сектору $^{10)}$. Разумеется, в обоих случаях дуальность меняет местами электрический и магнитный потенциалы. В КТП Люттингера мы получим

$$
Z_{\text {Lutt }}^{(r)}[\alpha, A]=Z_{\text {Lutt }}^{(0)}\left[\alpha^{-1}, \frac{i A^{*}}{\alpha}+b_{r}\right] \exp \left(\frac{1}{2 \pi \alpha} \int A^{2}\right),
$$

где $b_{r}$ соответствует магнитному потоку поля $r \Phi_{0} / 2$. Это показывает, что данный краевой заряд исходной теории становится магнитным потоком в дуальной теории.

В операторном подходе к КТП Люттингера производная исходного поля по пространственной координате оказывается плотностью заряда. Таким образом, бозонное поле может быть интерпретировано как пространственная деформация. Режим сильной связи - это режим слабых флуктуаций плотности заряда. С другой стороны, в дуальной теории производная дуального поля совпадает с электрическим током. Режим сильной связи в исходной теории отвечает сильным флуктуациям в дуальной теории.

10)Эта теория известна как $S U(2)$-модель Весса-Зумино-Виттена на уровне 1. 
Наконец, исследуем структуру пространства модулей КТП Люттингера. Для модулярно-инвариантных $c=1$ КТП это пространство было описано в восьмидесятых годах (см. обзор [24]). В нашем случае пространство модулей устроено более просто.

При нулевом магнитном поле эффективные теории задаются точками на прямой $\alpha>0$. Мы ишем "маргинальные" интегрируемые операторы. Каждый такой оператор порождает прямую линию орбифолдов в модулярно-инвариантном случае [27]. Однако это не так в случае КТП Люттингера: поиск операторов с конформными размерностями $(1,1)$ дает $n m=0$ и $n^{2} \alpha+m^{2} / 4 \alpha=2$. Кроме того, условие интегрируемости, которое основывается только на правилах слияния в бозонной модели, требует, чтобы оператор, который не присутствует в спектре КТП Люттингера, имел бы $n=0$ и $m=1$. Учет магнитного поля также делает невозможным существование таких операторов, т.к. нет операторов с конформными размерностями $(1,1)$ при всех значениях $\chi$.

Итак, единственными деформационными параметрами КТП Люттингера являются сила взаимодействия $\alpha$, магнитный поток $\chi$ и внешний потенциал. Разумеется, имеются такие значимые возмушения, которые выводят теорию из класса КТП. Например, член $\psi_{R}^{\dagger} \psi_{L}+\psi_{L}^{\dagger} \psi_{R}$ соответствует $\cos \left(2 R^{-1} \varphi\right)$ и становится сушественным при $\alpha>1 / 2$ и при нулевом магнитном поле. Этот оператор соответствует массивным возмушениям в модели Тирринга [58]. Можно также интересоваться другими операторами, отвечающими UMKLAPP-процессам, таким как $\left(\psi_{L}^{\dagger} \psi_{R}\right)^{2}$, которые соответствуют переносу импульса $-4 k_{F}$. Этот оператор возникает в модели решеточных фермионов (см. приложение A), он ответствен за фазовый переход XXZ-антиферромагнитной цепочки спина 1/2 из безмассовой фазы в массивную фазу Изинга при росте анизотропии. Этот оператор становится существенным при $\alpha>2$.

Аналогичные критерии используются при исследовании резонансного туннелирования ЖЛ через примесь. В этой проблеме полная плотность (включающая компоненты фурье-разложения $2 p k_{\mathrm{F}}$ ) взаимодействует с локальным потенциалом $U(\sigma)$, локализованным вблизи некоторого значения $\sigma$ :

$$
H_{\mathrm{imp}}=\int U(\sigma) \rho_{\mathrm{total}}(\sigma) d \sigma
$$

(см. [104] и [82, раздел IV.Е], а также непертурбативные результаты, полученные методом анзаца Бете [83-86] или в формфакторном подходе [87]).

\section{5. Примеры}

9.5.1. Жидкость Ферми. Основной пример отвечает значению $\alpha=1$, т.е. $p=q=$ 1. Эффективная КТП - это $\mathbb{Z} / 4 \mathbb{Z}$-теория, имеюшая четыре базисных примарных поля. Поле $\lambda=2$ имеет размерность $1 / 2$ и отвечает фермиону Дирака. В данном примере эти поля совпадают с фермионами Люттингера и с краевыми фермионами, обсуждаемыми в предыдущем разделе. Поля $\lambda= \pm 1$ имеют размерность $1 / 8$ и являются твистованными полями. Поле $\lambda=0$ - постоянное.

Статистическая сумма теории Дирака в зарядовом секторе $a$ задается формулой

$$
Z_{\mathrm{F}}^{(a)}(\tau, \bar{\tau})=\left|\chi_{a}(\tau)+\chi_{a+2}(\tau)\right|^{2}=\left|\varphi_{a}^{(+)}(\tau)\right|^{2} .
$$


Здесь $\omega=1$ и статистические суммы факторизуются, как и ожидалось, поскольку они являются квадратами модулей фермионных детерминантов [24]. Явные вычисления дают следуюшие формулы:

$$
\begin{aligned}
& \varphi_{0}^{(+)}=\chi_{0}+\chi_{2}=\vartheta_{3} / \eta \\
& \varphi_{1}^{(+)}=\chi_{1}+\chi_{3}=\vartheta_{2} / \eta \\
& \varphi_{0}^{(-)}=\chi_{0}-\chi_{2}=\vartheta_{4} / \eta \\
& \varphi_{1}^{(-)}=\chi_{1}-\chi_{3}=i \vartheta_{1} / \eta
\end{aligned}
$$

которые задают соответствие между $\mathbb{Z} / 4 \mathbb{Z}$-формулировкой данной модели и классическими тета-функциями. Статистическая сумма с $a=1$ есть $\left|\vartheta_{2} / \eta\right|^{2}$, что, в свою очередь, не что иное, как статистическая сумма $Z_{\mathrm{PA}}(\tau)$. Этот результат физически естествен т.к. добавление дополнительного электрона к свободной теории Дирака в АА-секторе эквивалентно рассмотрению этой теории в РА-секторе. Очевидно, что рассматриваемые здесь заряженные возмушения суть свободные электроны.

9.5.2. Лавлиновская жидкость Холла в точке общего положения. Здесь мы положим $\nu=1 / q$, и тогда $\alpha=q$ будет нечетным целым числом. Мы можем выбрать $p=1$ и сразу найти, что $\omega \equiv-1(\bmod 2 q)$. Эффективная $\mathrm{KT \Pi -это} \mathbb{Z} / 4 q \mathbb{Z}$-теория поля. Ее статистическая сумма может быть выражена через обобщенные характеры

$$
Z_{\mathrm{Lutt}}^{(a / q)}(\tau, \bar{\tau})=\sum_{\lambda \in \mathbb{Z} / q \mathbb{Z}} \varphi_{2 \lambda+a}^{(+)}(\tau) \overline{\varphi_{-2 \lambda+a}^{(+)}(\tau)}
$$

Все состояния, отвечающие $\varphi_{2 \lambda+a}^{(+)} \overline{\varphi_{-2 \lambda+a}^{(+)}}$, имеют левые и правые заряды, определенные с точностью до целых квантов заряда формулой

$$
\left(\frac{a}{2 q}+\frac{\lambda}{q}, \frac{a}{2 q}-\frac{\lambda}{q}\right)
$$

Заряд $a / q$ лавлиновского возмушения поделен поровну между двумя краями. Статистическая сумма периодична по $a$ с периодом $2 q$. Множитель 2 имеет простое объяснение: только в этом случае вклад в заряд на каждом краю будет целым.

Проиллюстрируем эти соображения в случае $\nu=1 / 3$ : эффективная теория есть $\mathrm{KT \Pi} \mathrm{с} \mathrm{полями} \mathbb{Z} / 12 \mathbb{Z}$. В ней имеются двенадцать примарных полей по отношению к подалгебре $\mathcal{A}_{+}$. В секторах с четным $а$ перенормированный фермион Люттингера соответствует полям $\chi_{4} \overline{\chi_{2}}$ и $\chi_{10} \overline{\chi_{8}}$, которые имеют конформные размерности $(h, \bar{h})=$ $(2 / 3,1 / 6)$ (и, следовательно, спин $1 / 2)$. Сохраняюшийся ток спина $3 / 2$ соответствует краевым фермионам. Обменные свойства двухточечной функции задаются функцией $(z-\xi)^{3}$ в точности, как для лавлиновской волновой функции. 


\section{0. ЗАКЛЮЧЕНИЕ}

В этой статье мы дали подробное описание эффективной КТП бесспиновой ЖЛ в бесшелевом режиме. Наше исследование верно при конечных размерах системы и при конечных температурах. Статистическая сумма и корреляционные функции плотностей заряда и тока вычислены при всех значениях константы связи в рамках некиральной схемы бозонизации. Сохраняющиеся токи порождают алгебру симметрий для всех КТП Люттингера. Их корреляционные функции позволяют найти функцию отклика системы и постоянные токи, индуцированные внешним магнитным потоком. Примарные по отношению к этой $\widehat{U(1)}_{R} \times \widehat{U(1)}_{L}$-симметрии поля суть вершинные операторы, чьи корреляционные функции точно вычисляются для случая КТП Люттингера.

Используя это описание КТП Люттингера, мы показали, как восстановить физику краевых возмущений дробной квантовой жидкости Холла на кольце. Заполняющая фракция простым образом связана с люттингеровским параметром взаимодействия, который играет роль модулей в КТП Люттингера. Решение КТП Люттингера было обобшено на твистованные секторы при описании краевых возмущений на фоне заданного объемного возмушения. Для КТП Люттингера с центральным зарядом $c=1$ мы обнаружили только лавлиновскую жидкость $\left(\nu^{-1} \in 2 \mathbb{N}+1\right)$, однако расширение нашего анализа на случай нескольких бозонных полей позволяет рассмотреть и другие заполняющие фракции ${ }^{11)}$. Оказывается, что КТП Люттингера, описывающие краевые возмушения ДКЭХ-жидкости, являются РКТП: в них имеются дополнительные сохраняющиеся токи, которые порождают алгебру расширенных симметрий, столь богатую, что гильбертово пространство состояний разлагается в конечную прямую сумму неприводимых представлений. Для ДКЭХ-жидкостей Лавлина эта алгебра порождается операторами рождения-уничтожения краевых фермионов. Это дает простую и красивую картину краевых возмущений в жидкостях Лавлина, все эти возмушения могут быть получены из конечного числа базисных возмущений либо с помощью добавления или вычитания фермионов, локализованных на левом или на правом краю, либо с помощью нейтральных деформаций, индуцированных $U(1)$-токами.

Формализм, развитый в данной статье, теперь может быть использован для исследования других аспектов одномерных сильно взаимодействуюших систем, таких как проблемы примесей (для описания которых большая часть работы уже проделана), взаимодействие круга Люттингера или цилиндра Холла с различными внешними системами типа фононов, квантованного электромагнитного поля, туннельных переходов, других систем Люттингера и т.д. В частности, результаты нашей статьи легко обобшаются на случай спаривания $N$ моделей Люттингера. Новым будет появление нескольких скоростей (приводящее к разделению спина и заряда для электронов спина $1 / 2$ ). При выделенных значениях констант связи можно получить РКТП, которая в некоторых случаях может соответствовать теориям Фрелиха-Керлера и Тирана (см. $[105,106]$ и ссылки в этих работах или $[107,108])$. Вычисления при конечных размерах и конечной температуре позволяют исследовать такие многочисленные эффекты, как дискретность зарядов, явления интерференции и то, как физика модели меняется с ростом температуры.

11) Работа в процессе подготовки. 
Другие сильно взаимодействуюшие системы также могут быть описаны с помошью тех же приемов, хотя структуры эффективного гильбертова пространства могут отличаться. Наиболее естественным примером таких систем является XXZ-цепочка спинов $1 / 2$ в своем безмассовом режиме ${ }^{12)}$. Введение беспорядка при рассеянии назад может приводить к различным физическим картинам, таким как локализация. Однако это трудная задача, решение которой конформными методами кажется весьма нетривиальным. Возможно, исследование симметрий квантовых групп в массивной фазе или использование квантового метода обратной задачи рассеяния может помочь в понимании перехода из бесшелевого режима в режим изолятора и его связи с влиянием эффектов, обусловленных конечными размерами образца.

Благодарности. Мы благодарны Б. Дуко за многочисленные интересные и стимулируюшие замечания, вопросы и предложения при выполнении этой работы. Мы также благодарны М. Бауэру, М. Блау и $Ф$. Дельдюку за полезные обсуждения роли тождеств Уорда в применении к бозонизации, а А. Капелли и Д. Карпентьеру за проясняющие объяснения и ссылки, касающиеся дробного квантового эффекта Холла. С. Дюзюель помог прояснить часть текста, посвяшенную описанию действия сдвигов по голономиям на статистические суммы. Мы искренне благодарны А. Изергину за частные обсуждения XY-цепочки спина $1 / 2$. Часть этой работы была сделана во время визита П. Деджованни и Р. Мелина в Исследовательский институт NEC в Принстоне. Во время этого визита авторы имели приятную возможность вести обсуждения с Б. Альтшулером, О. Агамом, А. Андреевым, И. Смоляренко, П. Чандрой, Н. Вингрином и С. Тангом, которые также снабдили нас многими полезными ссылками. Часть этой работы была также выполнена Р. Мелином в Интернациональной школе высших исследований (SISSA) в Триесте. Наконец, М. Фабрицио, М. Флор, Р. Стора и Дж. Земба снабдили нас многочисленными полезными ссылками.

Работа П. Деджованни поддержана грантом TMR FMRX-CT96-0012, а работа P. Мелина - грантом UPR 5001 от CNRS.

\section{ГАМИЛЬТОНИАНЫ В ИНФРАКРАСНОМ ПРЕДЕЛЕ \\ ПРИЛОЖЕНИЕ А}

В этом приложении мы напомним вывод гамильтониана одномерного фермионного газа в инфракрасном пределе. Начнем со свободного гамильтониана, а затем рассмотрим случай со взаимодействием.

\section{А.1. Свободный гамильтониан}

В начале раздела 2 мы объяснили, как линеаризовать дисперсионное соотношение меж ду двумя точками Ферми. Выведем выражение $(2.1)$ гамильтониана Дирака $H^{(0)}$ с помошью право- и леводвижушихся полей. Это хорошо известная процедура, которую мы воспроизведем из педагогических соображений.

\footnotetext{
12) Деджованни и Дюзюэль, работа в процессе подготовки.
} 
A.1.1. Решеточные фермионы. Начнем с гамильтониана вторичноквантованных решеточных фермионов

$$
H^{(0)}=\frac{t}{2} \sum_{n=1}^{N}\left(c_{n}^{+} c_{n+1}+c_{n+1}^{+} c_{n}\right)
$$

с циклическими граничными условиями. Это также гамильтониан фермионов Йордана-Вигнера антиферромагнитной XX0-спиновой цепочки с обменной постоянной $t=J$. Дисперсионное соотношение имеет вид $\epsilon(k)=t \cos (k)$. Непрерывный предел получает-

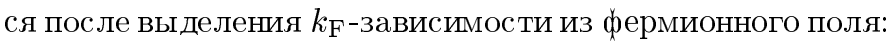

$$
c_{n}=\sqrt{\frac{a}{2}}\left(e^{i k_{\mathrm{F}} \sigma} \psi_{R}(\sigma)+e^{-i k_{\mathrm{F}} \sigma} \psi_{L}(\sigma)\right)
$$

где $\psi_{R, L}(\sigma)$ - медленно меняюшиеся поля, зависящие от переменной $\sigma=n a$. Для безразмерной постоянной $c_{n}$ поля $\psi_{R}$ и $\psi_{L}$ имеют скейлинговую размерность $1 / 2$. Более того, когда $\sigma$ становится непрерывной переменной, канонические антикоммутационные соотношения $\left\{\psi_{R, L}^{+}(\sigma), \psi_{R, L}\left(\sigma^{\prime}\right)\right\}=\delta\left(\sigma-\sigma^{\prime}\right)$ будут совместными с соотношениями для решеточных фермионов, имеющими вид $\left\{c_{n}^{+}, c_{m}\right\}=\delta_{n, m}$, если мы постулируем следуюшую связь между непрерывной и решеточной дельта-функциями: $\delta\left(\sigma-\sigma^{\prime}\right)=\delta_{n, n^{\prime}} / a$.

Разлагая (А.1) в терминах право- и леводвижушихся полей уравнения (А.2), получим

$$
2 H^{(0)}=v_{\mathrm{F}} \int_{0}^{L} d \sigma\left(\psi_{R}^{+}(\sigma) i \partial_{\sigma} \psi_{R}(\sigma)-\psi_{L}^{+}(\sigma) i \partial_{\sigma} \psi_{L}(\sigma)\right),
$$

где $v_{\mathrm{F}}=\partial \epsilon\left(k_{\mathrm{F}}\right) / \partial k=a t \sin \left(k_{\mathrm{F}} a\right)$. Правая часть уравнения (А.3) - это гамильтониан теории Дирака. При выводе (А.3) мы, во-первых, пренебрегли быстро меняющимися полями с множителями $\exp \left( \pm 2 i k_{\mathrm{F}} \sigma\right)$, т.к. эти процессы имеют пренебрежимо малую вероятность, а $2 k_{\mathrm{F}}$ не является вектором обратной решетки. В присутствии димеризации (т.е. при $t_{2 i}=t(1-\delta)$ и $\left.t_{2 i+1}=t(1+\delta)\right)$ эти процессы не будут пренебрежимо ма-

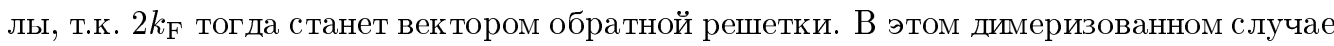
$2 k_{\mathrm{F}}$-процессы ответственны за появление массовой шели. Во-вторых, мы рассматривали переменную $\sigma$ как непрерывную, в-третьих, в разложении $\psi_{R, L}^{+}(\sigma+a)=\psi_{R, L}^{+}(\sigma)+$ $a \partial_{\sigma} \psi_{R, L}^{+}(\sigma)$ пренебрегли производными старших порядков, которые не дают вклада в инфракрасном пределе, и, в-четвертых, опустили постоянную энергии наинизшего состояния.

A.1.2. Нерелятивистские фермионы. Заметим, что тот же анализ может быть выполнен, если начинать с микроскопического гамильтониана

$$
H^{(0)}=\int_{0}^{L} \Psi^{+}(\sigma) \frac{\Delta^{2}}{2 m} \Psi(\sigma) d \sigma .
$$

Фермионное поле $\Psi(\sigma)$ относится к киральным полям как

$$
\Psi(\sigma)=\frac{1}{\sqrt{2}}\left(e^{i k_{\mathrm{F}} \sigma} \psi_{R}(\sigma)+e^{-i k_{\mathrm{F}} \sigma} \psi_{L}(\sigma)\right),
$$

а низкоэнергетический эффективный гамильтониан имеет вид (А.3).

3 Теоретическая и математическая физика, т. 117, № 1, 1998 г. 


\section{А.2. Решеточная модель со взаимодействиями $g_{2}$ и $g_{4}$.}

В этом разделе мы покажем, что, как правило, гамильтониан (2.15), содержаший лишь взаимодействия $g_{2}$ и $g_{4}$, не является наиболее обшим низкоэнергетическим гамильтонианом одномерных бесспиновых фермионов. Возникновение дополнительных взаимодействий может быть обусловлено возможностью UMKLAPP-процессов в присутствии эффективной решетки. Эти UMKLAPP-процессы отвечают событиям рассеяния, в которых переданный импульс пропорционален обратному вектору решетки. Эти члены порождают взаимодействия типа Гросса-Невё, которые могут радикально изменить физику модели. Например, в XXZ-цепочке эти UMKLAPP-процессы ответственны за поток ренормгруппы к фиксированной точке массивной модели Изинга при $J_{z}>J_{x}=J_{y}$. Этими членами можно пренебречь при заполнении уровней, далеком от половинного, поскольку при выполнении этого условия процессы рассеяния на фоне моря Ферми не могут переносить импульс, принадлежащий обратной решетке. Однако, если выбрать очень специальные условия, эти члены могут исчезать даже при половинном заполнении и тогда физика больших расстояний будет в точности совпадать с физикой, описанной в данной статье.

Более конкретно мы рассматриваем решеточные бесспиновые одномерные фермионные системы с членом перескока (А.1) и с членом взаимодействия, включающим взаимодействия с ближайшими и следуюшими за ближайшими соседними узлами:

$$
H^{(1)}(U, V)=U \sum_{n=1}^{N} c_{n}^{+} c_{n} c_{n+1}^{+} c_{n+1}+V \sum_{n=1}^{N} c_{n}^{+} c_{n} c_{n+2}^{+} c_{n+2} .
$$

Используя разложение (А.2) на быстро и медленно меняющиеся моды, мы получим

$$
\begin{aligned}
c_{n}^{+} c_{n}= & \frac{a}{2}\left(\psi_{R}^{+}(\sigma) \psi_{R}(\sigma)+\psi_{L}^{+}(\sigma) \psi_{L}(\sigma)+\right. \\
& \left.+e^{-2 i k_{\mathrm{F}} \sigma} \psi_{R}^{+}(\sigma) \psi_{L}(\sigma)+e^{2 i k_{\mathrm{F}} \sigma} \psi_{L}^{+}(\sigma) \psi_{R}(\sigma)\right) .
\end{aligned}
$$

Используя это последнее уравнение, мы получим низкоэнергетический гамильтониан взаимодействия

$$
\begin{aligned}
2 H^{(1)}(U, V)= & \frac{(U+V) v_{\mathrm{F}}}{2 t \sin \left(k_{\mathrm{F}} a\right)} \int_{0}^{L}\left(\mathcal{J}^{2}(\sigma)+\overline{\mathcal{J}}^{2}(\sigma)\right) d \sigma+\frac{(U+V) v_{\mathrm{F}}}{t \sin \left(k_{\mathrm{F}} a\right)} \int_{0}^{L} \mathcal{J}(\sigma) \overline{\mathcal{J}}(\sigma) d \sigma+ \\
& +\left\{\frac{v_{\mathrm{F}} e^{-2 i k_{\mathrm{F}} a}\left(U+V e^{-2 i k_{\mathrm{F}} a}\right)}{2 t \sin \left(k_{\mathrm{F}} a\right)} \int e^{-4 i k_{\mathrm{F}} \sigma}\left(\psi_{R}^{+}(\sigma) \psi_{L}(\sigma)\right)^{2}+\text { h.c. }\right\},
\end{aligned}
$$

где токи $\mathcal{J}$ и $\overline{\mathcal{J}}$ определены в пункте 2.1.1. Последний член этого гамильтониана соответствует UMKLAPP-процессам с переносом импульса $4 k_{\mathrm{F}}$. При половинном заполнении $\left(k_{\mathrm{F}}=\pi / 2 a\right)$ эти члены равны нулю, если $U=V$. При этих условиях остаюшийся гамильтониан взаимодействия включает в себя только взаимодействия $g_{2}$ и $g_{4}$ :

$$
2 H^{(1)}(U, U)=\frac{U v_{\mathrm{F}}}{t} \int_{0}^{L}\left(\mathcal{J}^{2}(\sigma)+\overline{\mathcal{J}}^{2}(\sigma)\right) d \sigma+\frac{2 U v_{\mathrm{F}}}{t} \int_{0}^{L} \mathcal{J}(\sigma) \overline{\mathcal{J}}(\sigma) d \sigma .
$$




\section{ЯВНЫЕ ВЫЧИСЛЕНИЯ В ТЕОРИИ ДИРАКА}

Здесь мы вычислим свободные фермионные статистические суммы при наличии магнитного потока и химического потенциала. Отождествление фермионных детерминантов с бозонными статистическими суммами также заслуживает более подробного обсуждения. Упор делается на модулярные свойства всех статистических сумм и на их поведение при различных сдвигах магнитного потока и химического потенциала.

\section{В.1. Антипериодические фермионы, взаимодействующие с магнитным полем и химическим потенциалом}

В.1.1. Операторные вычисления с фермионами. Вычисление статистической суммы в теории Дирака в присутствии магнитного потока и химического потенциала проводится в рамках дзета-функциональной процедуры перенормировки. Вклад левых и правых мод в вакуумную энергию имеет вид

$$
E_{R}=-\frac{\pi v_{\mathrm{F}}}{L}\left(\frac{1}{12}-(\lambda+a)^{2}\right), \quad E_{L}=-\frac{\pi v_{\mathrm{F}}}{L}\left(\frac{1}{12}-(\lambda-a)^{2}\right),
$$

где $\lambda=-L \mu / 2 \pi v_{\mathrm{F}}$. Вклад возмушения легко вычисляется с помошью частично-дырочной картины, а вводя эффективную переменную $y=e^{\beta \mu}$, мы получим окончательный ответ

$$
\begin{aligned}
Z_{\mathrm{AA}}^{(0)}(a, \mu)= & q^{\frac{(\lambda+a)^{2}}{2}} \bar{q}^{\frac{(\lambda-a)^{2}}{2}}(q \bar{q})^{-\frac{1}{24}} \prod_{n=0}^{+\infty}\left(1+y q^{n+a+1 / 2}\right)\left(1+y^{-1} q^{n-a+1 / 2}\right) \times \\
& \times \prod_{n=0}^{+\infty}\left(1+y \bar{q}^{n-a+1 / 2}\right)\left(1+y^{-1} \bar{q}^{n+a+1 / 2}\right) .
\end{aligned}
$$

Сразу отметим, что вклад $(q \bar{q})^{\lambda^{2} / 2}$ отвечает объемному вкладу в свободную энергию. Все прочие вклады поступают от энергетических уровней, которые ведут себя при масштабном преобразовании как $L^{-1}$. Таким образом, мы опустим объемный вклад, который будет пониматься как нулевая энергия. Вклад $(q / \bar{q})^{\lambda a}$, который преобразуется как $L^{0}$, будет также опускаться. Таким образом, окончательное выражение для статистической суммы примет вид

$$
\begin{aligned}
Z_{\mathrm{AA}}(a, \mu)= & (q \bar{q})^{\frac{a^{2}}{2}-\frac{1}{24}} \prod_{n=0}^{+\infty}\left(1+y q^{n+a+1 / 2}\right)\left(1+y^{-1} q^{n-a+1 / 2}\right) \times \\
& \times \prod_{n=0}^{+\infty}\left(1+y \bar{q}^{n-a+1 / 2}\right)\left(1+y^{-1} \bar{q}^{n+a+1 / 2}\right) .
\end{aligned}
$$

С помощью тождества тройного произведения Якоби это выражение может быть переписано следующим образом:

$$
Z_{\mathrm{AA}}(a, \mu)=\frac{1}{|\eta(\tau)|^{2}} \sum_{(m, \bar{m}) \in \mathbb{Z}^{2}} q^{\frac{1}{2}(m+a)^{2}} \bar{q}^{\frac{1}{2}(\bar{m}-a)^{2}} y^{m+\bar{m}} .
$$


Полезно сравнить эти выражения с ответами, полученными Саксом и Випфом в [63]. Для вычисления они использовали фермионный функциональный интеграл, с помощью которого также исследовалась дзета-функция для спектра оператора Дирака. Сакс и Випф также сравнивали свой результат с результатом операторных вычислений, приведенных в приложении к основному тексту своей статьи. Их результаты совпадают с нашими ${ }^{13)}$.

B.1.2. Сравнение фермионных и бозонных результатов в секторе АА. Сравним функцию $Z_{\mathrm{AA}}(a, \mu)$ с функцией $K_{\mathrm{AA}}(a, \mu)$, определенной следующим образом:

$$
\begin{aligned}
K_{\mathrm{AA}}(a, \mu)= & \frac{1}{2}\left(Z_{[0,0]}\left(a,-\frac{i \mu \beta}{2 \pi}\right)+Z_{\left[\frac{1}{2}, 0\right]}\left(a,-\frac{i \mu \beta}{2 \pi}\right)+\right. \\
& \left.+Z_{\left[0, \frac{1}{2}\right]}\left(a,-\frac{i \mu \beta}{2 \pi}\right)-Z_{\left[\frac{1}{2}, \frac{1}{2}\right]}\left(a,-\frac{i \mu \beta}{2 \pi}\right)\right) .
\end{aligned}
$$

Это выражение имеет вид (см. раздел 3.1 )

$$
K_{\mathrm{AA}}(a, \mu)=\frac{1}{|\eta(\tau)|^{2}} \sum_{\substack{(l, k) \in \mathbb{Z}^{2} \\ k \equiv l(\bmod 2)}} y^{l} q^{\frac{1}{8}(l+k+2 a)^{2}} \bar{q}^{\frac{1}{8}(l-k-2 a)^{2}} .
$$

Таким образом, с помощью $m=(l+k) / 2$ и $\bar{m}=(l-k) / 2$ можно легко восстановить правую часть уравнения (В.4), т.к. $m$ и $\bar{m}$ принадлежат теперь $\mathbb{Z}^{2}$ из-за условия четности $l \equiv k(\bmod 2)$. Таким образом, окончательное соотношение между фермионной дзета-перенормированной статистической суммой и бозонной статистической суммой ${ }^{14}$ ) выглядит следуюшим образом:

$$
Z_{\mathrm{AA}}^{(0)}(a, \mu)=(q \bar{q})^{\frac{\lambda^{2}}{2}}\left(\frac{q}{\bar{q}}\right)^{\lambda a} K_{\mathrm{AA}}(a, \mu) .
$$

Это доказывает формулу бозонизации для АА-сектора в присутствии магнитного потока и однородного химического потенциала. Обратимся теперь к АР-сектору.

B.1.3. Сектор АP. Этот сектор можно получить из АА-сектора, меняя $y$ на $-y$ в частично-дырочном вкладе в $Z_{\mathrm{AA}}$. Вакуумный вклад не меняется. Таким образом, мы получим

$$
Z_{\mathrm{AP}}^{(0)}(a, \mu)=(q \bar{q})^{\frac{\lambda^{2}}{2}}\left(\frac{q}{\bar{q}}\right)^{\lambda a} K_{\mathrm{AA}}\left(a, \mu+\frac{1 \pi}{\beta}\right)
$$

\section{В.2. Сдвиги и свойства модулярной инвариантности}

Продолжим исследование фермионных детерминантов. Будем сдвигать $a$ и химический потенциал (или эквивалентно $b$ ). Для этого, во-первых, конкретизируем влияние таких сдвигов на бозонные статистические суммы, а затем на фермионные статистические суммы.

\footnotetext{
13) Авторы [63] предполагали, что $\tau$ вещественно, и тоже опускали объемный вклад в энергию.

${ }^{14)}$ Мы оставили объемные множители для удобства, в дальнейшем они будут опушены.
} 
В.2.1. Влияние сдвигов на бозонные статистические суммы. Сначала будем сдвигать бозонные выражения. Введем три новые бозонные функции:

$$
\begin{aligned}
K_{\mathrm{AP}}(a, \mu) & =K_{\mathrm{AA}}\left(a, \mu+\frac{i \pi}{\beta}\right)= \\
& =\frac{1}{|\eta(\tau)|^{2}} \sum_{(m, \bar{m}) \in \mathbb{Z}^{2}}(-y)^{m+\bar{m}} q^{\frac{1}{2}(m+a)^{2}} \bar{q}^{\frac{1}{2}(\bar{m}-a)^{2}}, \\
K_{\mathrm{PA}}(a, \mu) & =K_{\mathrm{AA}}\left(a+\frac{1}{2}, \mu\right)= \\
& =\frac{1}{|\eta(\tau)|^{2}} \sum_{(m, \bar{m}) \in \mathbb{Z}^{2}} y^{m+\bar{m}+1} q^{\frac{1}{2}(m+a+1 / 2)^{2}} \bar{q}^{\frac{1}{2}(\bar{m}-a+1 / 2)^{2}}, \\
K_{\mathrm{PP}}(a, \mu) & =K_{\mathrm{PP}}\left(a+\frac{1}{2}, \mu+\frac{i \pi}{\beta}\right)= \\
& =\frac{1}{|\eta(\tau)|^{2}} \sum_{(m, \bar{m}) \in \mathbb{Z}^{2}}(-y)^{m+\bar{m}+1} q^{\frac{1}{2}(m+a+1 / 2)^{2}} \bar{q}^{\frac{1}{2}(\bar{m}-a+1 / 2)^{2}} .
\end{aligned}
$$

Конечно, эти статистические суммы могут быть также выражены как

$$
\begin{aligned}
K_{\mathrm{AP}}(a, \mu) & =\frac{1}{2}\left(Z_{[0,0]}+Z_{[0,1 / 2]}-Z_{[1 / 2,0]}+Z_{[1 / 2,1 / 2]}\right), \\
K_{\mathrm{PA}}(a, \mu) & =\frac{1}{2}\left(Z_{[0,0]}-Z_{[0,1 / 2]}+Z_{[1 / 2,0]}+Z_{[1 / 2,1 / 2]}\right), \\
K_{\mathrm{PP}}(a, \mu) & =\frac{1}{2}\left(Z_{[0,0]}-Z_{[0,1 / 2]}-Z_{[1 / 2,0]}-Z_{[1 / 2,1 / 2]}\right),
\end{aligned}
$$

что легко следует из очевидных свойств функций $Z_{\left[\epsilon, \epsilon^{\prime}\right]}(a, b)$ :

$$
\begin{aligned}
& Z_{\left[\epsilon, \epsilon^{\prime}\right]}\left(a+\frac{1}{2}, b\right)=e^{2 \pi i \epsilon^{\prime}} Z_{\left[\epsilon, \epsilon^{\prime}\right]}(a, b), \\
& Z_{\left[\epsilon, \epsilon^{\prime}\right]}\left(a, b+\frac{1}{2}\right)=e^{2 \pi i \epsilon} Z_{\left[\epsilon, \epsilon^{\prime}\right]}(a, b) .
\end{aligned}
$$

B.2.2. Свойства совместности. Интересно исследовать поведение системы при последовательных сдвигах $a$ и $b$ и свойства статистических сумм и формул бозонизации при модулярных преобразованиях.

Более точно, введем векторные обозначения, которые одновременно включают в себя четыре фермионных сектора и четыре бозонных сектора. В этих обозначениях некиральная бозонизация может быть выражена следующим образом (верно для вещественньх $(a, b))$ :

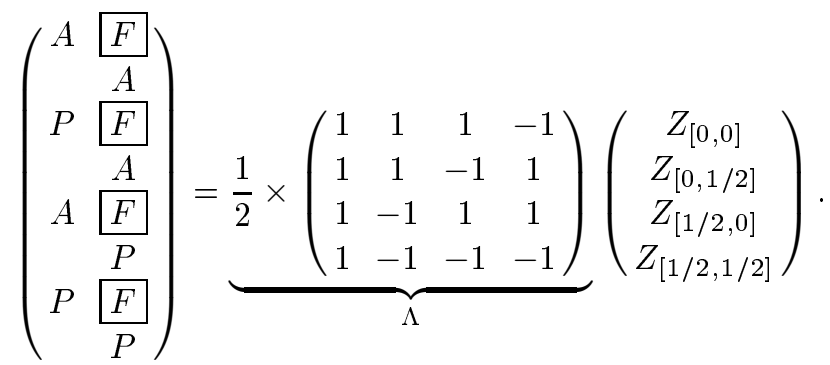


В этом подходе сдвиги на $1 / 2$ величин $a$ и $b$ представлены матрицами, которые мы обозначим через $B_{(a, b)}$ для бозонов ${ }^{15)}$ и $F_{(a, b)}$ для фермионов. Тогда, если $\Lambda$ обозначает матрицу, связываюшую бозонные статистические суммы с фермионными, мы получим

$$
F_{(a)} \times \Lambda=\Lambda \times B_{(a)} \quad \text { и } \quad F_{(b)} \times \Lambda=\Lambda \times B_{(b)} .
$$

Легко проверить, что эти соотношения совместны с модулярными свойствами бозонных статистических сумм $Z_{\left[\epsilon, \epsilon^{\prime}\right]}$. Модулярные преобразования $S$ и $T$ могут быть представлены $4 \times 4$-матрицами бозонных и фермионных статистических сумм. Более явно, матрищы этих модулярных преобразований имеют вид

$$
S^{(B)}=\left(\begin{array}{llll}
1 & 0 & 0 & 0 \\
0 & 0 & 1 & 0 \\
0 & 1 & 0 & 0 \\
0 & 0 & 0 & 1
\end{array}\right) \quad \text { и } \quad S^{(F)}=\left(\begin{array}{llll}
1 & 0 & 0 & 0 \\
0 & 0 & 1 & 0 \\
0 & 1 & 0 & 0 \\
0 & 0 & 0 & 1
\end{array}\right),
$$

а для $T-$

$$
T^{(B)}=\left(\begin{array}{cccc}
1 & 0 & 0 & 0 \\
0 & 1 & 0 & 0 \\
0 & 0 & 0 & 1 \\
0 & 0 & 1 & 0
\end{array}\right) \quad \text { и } \quad T^{(F)}=\left(\begin{array}{llll}
0 & 1 & 0 & 0 \\
1 & 0 & 0 & 0 \\
0 & 0 & 1 & 0 \\
0 & 0 & 0 & 1
\end{array}\right)
$$

Разумеется, мы получим

$$
T^{(B)} \times \Lambda=\Lambda \times T^{(F)} \quad \text { и } \quad S^{(B)} \times \Lambda=\Lambda \times S^{(F)},
$$

что выражает совместность $S L(2, \mathbb{Z})$-действия нашей матрицы бозонизации $\Lambda$ на статистические суммы. Затем мы также проверим, что сдвигающие матрищы совместны с матрицами модулярного преобразования:

$$
\left\{\begin{array} { l } 
{ S ^ { ( B ) } \cdot B _ { ( a ) } = B _ { ( b ) } S ^ { ( B ) } , } \\
{ S \cdot B _ { ( b ) } = B _ { ( a ) } S , }
\end{array} \quad \text { и } \quad \left\{\begin{array}{l}
T^{(B)} B_{(a)}=B_{(a)} T^{(B)}, \\
T^{(B)} B_{(b)}=B_{(a)} B_{(b)} T^{(B)} .
\end{array}\right.\right.
$$

Похожие уравнения верны для $F_{(a, b)}$.

Условие совместности модулярных преобразований и матрицы бозонизации лиш частично фиксирует последнюю. Более точно, накладывая условие (В.22), мы получим, что матрица $\Lambda$ должна иметь следуюший вид:

$$
\Lambda=\left(\begin{array}{llll}
a & b & b & c \\
a & b & c & b \\
a & c & b & b \\
d & e & e & e
\end{array}\right)
$$

Формула бозонизации в РР-секторе оказывается не зависимой от формул бозонизаций в трех других фермионных секторах. Лиш одни уравнения (В.18) дают некоторые ограничения на $\Lambda$, как было ранее отмечено в работе [33]. В обозначениях (В.23) мы получим

15) Диагональная матрица с собственными значениями, задаваемыми уравнениями (В.15) и (В.16). 
$b=-c, e=c$ и $d=a$. Таким образом, $\Lambda$ зависит только от двух коэффициентов, что и следовало ожидать, поскольку $Z_{[0,0]}$ - модулярньй инвариант:

$$
\Lambda=\left(\begin{array}{cccc}
a & b & b & -b \\
a & b & -b & b \\
a & -b & b & b \\
a & -b & -b & -b
\end{array}\right)
$$

Отсюда формула бозонизации для фермионного сектора АА фиксирует формулы бозонизации во всех оставшихся секторах.

Однако эта красивая картина рушится при включении взаимодействия фермионов с химическим потенциалом или электрическим потенциалом. Такие потенциалы соответствуют мнимому твисту для граничных значений $b$. Однако, как заметили Сакс и Випф [63, раздел 3.1], вычисление дзета-перенормированной статистической суммы требует косвенных методов расчета. Дзета-функция оператора Дирака допускает хорошо определенное аналитическое продолжение с помошью формулы пересуммирования Пуассона лишь при вешественных $a$ и $b$ (относительно АА-сектора). Таким образом, введение химического потенциала приводит к необходимости аналитически продолжать полученный результат в предположении, что параметр $b$ комплексен. Однако в этом случае надо внимательно отнестись к вкладам, возникающим из части ответа, зависяшей от аналитического продолжения, т.е. из вкладов энергии вакуума.

Обратимся к секторам РА и РР, которые требуют некоторой осторожности, как мы покажем в следующем разделе.

\section{B.3. Секторы PA и PP}

Сдвиг в фермионных статистических суммах. Фермионные статистические суммы также могут быть вычислены явно. Вычисление в антипериодическом фермионном секторе уже было проделано, и в этом случае дзета-перенормировка вакуумной энергии дает

$$
\begin{aligned}
& E_{R}=-\frac{\pi v_{\mathrm{F}}}{L}\left((a+\lambda)^{2}+a+\lambda+\frac{1}{6}\right), \\
& E_{L}=-\frac{\pi v_{\mathrm{F}}}{L}\left((a-\lambda)^{2}+\lambda-a+\frac{1}{6}\right)
\end{aligned}
$$

Из этих вакуумных энергий вычисление следа по фермионному пространству Фока дает $Z_{\mathrm{PA}}$ как произведение левых и правых вкладов:

$$
\begin{aligned}
& Z_{\mathrm{PA}}^{(R)}(a, \mu)=q^{\frac{1}{2}\left((\lambda+a)^{2}-a-\lambda\right)+\frac{1}{12}} \prod_{n=0}^{+\infty}\left(1+y q^{n+a}\right)\left(1+y^{-1} q^{n+1-a}\right), \\
& Z_{\mathrm{PA}}^{(L)}(a, \mu)=\bar{q}^{\frac{1}{2}\left((\lambda-a)^{2}+a-\lambda\right)+\frac{1}{12}} \prod_{n=0}^{+\infty}\left(1+y \bar{q}^{n-a}\right)\left(1+y^{-1} \bar{q}^{n+1+a}\right) .
\end{aligned}
$$


Тождество Якоби переводит бесконечные произведения в степенные ряды:

$$
\begin{aligned}
Z_{\mathrm{PA}}^{(R)}(a, \mu) Z_{\mathrm{PA}}^{(L)}(a, \mu)= & (q \bar{q})^{\lambda^{2} / 2}\left(\frac{q}{\bar{q}}\right)^{\lambda a}(q \bar{q})^{-\lambda / 2} \times \\
& \times \sum_{(m, \bar{m}) \in \mathbb{Z}^{2}} y^{m+\bar{m}} q^{\frac{1}{2}(m+a-1 / 2)^{2}} \bar{q}^{\frac{1}{2}(\bar{m}-a-1 / 2)^{2}} .
\end{aligned}
$$

Сделав сдвиг $m \mapsto m+1$ и $\bar{m} \mapsto \bar{m}+1$ и учитьвая, что $(q \bar{q})^{-\lambda / 2}=y^{-1}$, окончательно получим

$$
Z_{\mathrm{PA}}^{(0)}(a, \mu)=(q \bar{q})^{\lambda^{2} / 2}(q \bar{q})^{\lambda a} \sum_{(m, \bar{m}) \in \mathbb{Z}^{2}} y^{m+\bar{m}+1} q^{\frac{1}{2}(m+a+1 / 2)^{2}} \bar{q}^{\frac{1}{2}(m-a+1 / 2)^{2}} .
$$

Таким образом, сравнение с бозонными выражениями дает

$$
Z_{\mathrm{PA}}(a, \mu)=(q \bar{q})^{\lambda^{2} / 2}(q \bar{q})^{\lambda a} K_{\mathrm{PA}}(a, \mu)
$$

Полезно теперь найти фермионную статистическую сумму в РР-секторе. В этом случае вакуумный вклад остается таким же, как и для статистической суммы в секторе РА. Нужно лишь перейти от $y$ к $-y$ во вкладах частиц и дырок в статистические суммы. Отсюда имеем

$$
\begin{aligned}
& Z_{\mathrm{PP}}^{(R)}(a, \mu)=q^{\frac{1}{2}\left((\lambda+a)^{2}-a-\lambda\right)+\frac{1}{12}} \prod_{n=0}^{+\infty}\left(1-y q^{n+a}\right)\left(1-y^{-1} q^{n+1-a}\right), \\
& Z_{\mathrm{PP}}^{(L)}(a, \mu)=\bar{q}^{\frac{1}{2}\left((\lambda-a)^{2}+a-\lambda\right)+\frac{1}{12}} \prod_{n=0}^{+\infty}\left(1-y \bar{q}^{n-a}\right)\left(1-y^{-1} \bar{q}^{n+1+a}\right) .
\end{aligned}
$$

Снова используя тождество тройного произведения Якоби, получим

$$
\begin{aligned}
Z_{\mathrm{PP}}^{(R)}(a, \mu) Z_{\mathrm{PP}}^{(L)}(a, \mu)= & (q \bar{q})^{\frac{1}{2}\left(\lambda^{2}-\lambda\right)}\left(\frac{q}{\bar{q}}\right)^{\lambda a} \times \\
& \times \sum_{(m, \bar{m}) \in \mathbb{Z}^{2}}(-y)^{m+\bar{m}} q^{\frac{1}{2}(m+a-1 / 2)^{2}} \bar{q}^{\frac{1}{2}(\bar{m}-a-1 / 2)^{2}}
\end{aligned}
$$

а те же сдвиги, что и раньше, дают

$$
\begin{aligned}
Z_{\mathrm{PP}}^{(0)}(a, \mu)= & (q \bar{q})^{\lambda^{2} / 2}\left(\frac{q}{\bar{q}}\right)^{\lambda a} \times \\
& \times \sum_{(m, \bar{m}) \in \mathbb{Z}^{2}}(-1)^{m+\bar{m}} y^{m+\bar{m}+1} q^{\frac{1}{2}(m+a+1 / 2)^{2}} \bar{q}^{\frac{1}{2}(\bar{m}-a+1 / 2)^{2}} .
\end{aligned}
$$

Это выражение можно сравнить с соответствуюшей бозонной статистической суммой

$$
Z_{\mathrm{PP}}^{(0)}(a, \mu)=-(q \bar{q})^{\lambda^{2} / 2}\left(\frac{q}{\bar{q}}\right)^{2} \mu
$$


Знак минус перед ней важен, хотя мы и не использовали РР-сектор в этой статье, а рассматривали в основном АА-сектор безмассовой модели Тирринга. Во-первых, подчеркнем, что полученные знаки должны гарантировать положительность статистических сумм $Z_{\mathrm{PP}}$ и $Z_{\mathrm{PA}}$. При нулевом магнитном поле старшие асимптотики при нулевой температуре следующие:

$$
\begin{aligned}
& Z_{\mathrm{PA}} \simeq(q \bar{q})^{\frac{1}{12}}\left(y+y^{-1}+2\right)=4(q \bar{q})^{\frac{1}{12}} \operatorname{ch}^{2}\left(\frac{\beta \mu}{2}\right) \\
& Z_{\mathrm{PP}} \simeq(q \bar{q})^{\frac{1}{12}}\left(y+y^{-1}-2\right)=4(q \bar{q})^{\frac{1}{12}} \operatorname{sh}^{2}\left(\frac{\beta \mu}{2}\right)
\end{aligned}
$$

в то время как $K_{\mathrm{PP}}$ имеет вид $2-y-y^{-1}=-\operatorname{sh}^{2}(\beta \mu / 2)$. Технически знак в выражении (В.35) возникает при удалении множителя $|y|$, идущего из вакуумных вкладов при построении функции $K_{\mathrm{PA}}$ и $K_{\mathrm{PP}}$. Более формальный способ это выразить состоит в том, что используются свойства аналитичности дзета-регуляризованных фермионных детерминантов и их поведение вблизи нулей.

Наконец отметим, что этот знак важен для нахождения правильной эффективной KТП на поверхности рода 1, которая описывает XXZ-модель конечного размера при конечной температуре ${ }^{16)}$.

\section{УСЛОВИЯ НОРМИРОВКИ ДЛЯ БОЗОННЫХ ФУНКЦИОНАЛЬНЫХ ИНТЕГРАЛОВ}

ПРИЛОЖКЕНИЕ С

\section{C.1. Нормировки}

Для некомпактифицированного бозонного поля $\varphi$ мера интегрирования $\mathcal{D}_{g}[\varphi]$ обычно (см. [24]) нормируется следующим образом:

$$
\int \mathcal{D}_{g}[\varphi] \exp \left(-\frac{g}{2 \pi} \int \varphi^{2}\right)=1
$$

Разложим $\varphi$ на постоянную часть $\varphi_{0}$ и флуктуируюшую часть $\widetilde{\varphi}$ с нулевым средним: $\varphi=\varphi_{0}+\widetilde{\varphi}$. Мы введем $\mathcal{D}_{\perp, g}-$ ограничение нашей меры на пространство функций с нулевым средним. Тогда уравнение (С.1) дает

$$
\int \mathcal{D}_{\perp, g}[\widetilde{\varphi}] \exp \left(-\frac{g}{2 \pi} \int \widetilde{\varphi}^{2}\right)=\sqrt{\frac{g \mathcal{A}}{2 \pi^{2}}} .
$$

На пространстве функций с нулевым средним меры $\mathcal{D}_{\perp, g}$ соотносятся одна с другой по формуле

$$
\mathcal{D}_{\perp, g}=\sqrt{\frac{g}{g^{\prime}}} \mathcal{D}_{\perp, g^{\prime}}
$$

что следует из уравнения (С.2).

\footnotetext{
16) Деджованни и Дюзюэль, работа в процессе подготовки.
} 
Для поля, компактифицированного на круг радиуса $R$, мы должны задать интегрирование по нулевой моде $\varphi_{0}=\mathcal{A}^{-1} \int \varphi$. Мы выбираем обычный объем $d \varphi_{0}$ на пространстве $\mathbb{R} / 2 \pi R \mathbb{Z}$. Это дает инстантонную сумму, а флуктуационная часть $\xi$ с нулевым средним остается прежней. Таким образом, мера интегрирования разлагается следующим образом:

$$
\mathcal{D}_{R, g}[\varphi]=\sum_{\text {instantons }} d \varphi_{0} \mathcal{D}_{\perp, g}[\xi]
$$

\section{С.2. Вклад флуктуаций}

Напомним, как вычислить вклад фллутуаций:

$$
Z_{\text {fluct }}=\int \mathcal{D}_{\perp, g}[\varphi] \exp \left(-\frac{g}{2 \pi} \int(d \widetilde{\varphi})^{2}\right)
$$

С помощью уравнения (C.2) эта статистическая сумма выражается в терминах детерминанта лапласиана на пространстве функций с нулевым средним:

$$
Z_{\text {fluct }}=\sqrt{\frac{g \mathcal{A}}{2 \pi^{2}}} \operatorname{Det}_{\perp}^{-1 / 2}(-\Delta)
$$

Простейший способ вычислить этот детерминант - использовать дзета-функциональную процедуру перенормировки. Из спектра лапласиана на торе получим

$$
Z_{\text {fluct }}=\frac{1}{\sqrt{2 \pi^{2} \mathcal{A}}} \frac{1}{|\eta(\tau)|^{2}},
$$

где $\eta$-функция Дедекинда имеет вид

$$
\eta(\tau)=e^{i \pi \tau / 12} \prod_{n=1}^{+\infty}\left(1-q^{n}\right) .
$$

Этот результат не зависит от $g$, как и следовало ожидать из якобиана (С.3).

\section{ЯВНЫЕ ВЫЧИСЛЕНИЯ В БОЗОННОЙ ТЕОРИИ}

ПРИЛОЖКЕНИЕ D

Напомним, как вычислить некоторые статистические суммы в теории свободного бозона, компактифицированного на окружность радиуса $R$. Функциональный интеграл задается уравнением (2.60). Чтобы его “определить", требуется задать ренормализацию для функциональных интегралов. Все детали этого содержатся в приложении С, а в данном приложении рассматриваются "инстантонные" вопросы, связанные с этим вычислением. 


\section{D.1. Инстантоны}

Мы ищем инстантонные решения с граничными условиями $\left[\epsilon, \epsilon^{\prime}\right]$. Каждое комплексное число $z$ может быть единственным образом записано как $z=x \omega_{1}+t \omega_{2}$, где $(x, t) \in \mathbb{R}^{2}$ определены

$$
\begin{aligned}
& x=\Re\left(\frac{z}{\omega_{1}}\right)-\frac{\Re(\tau)}{\Im(\tau)} \Im\left(\frac{z}{\omega_{1}}\right), \\
& t=\frac{\Im\left(z / \omega_{1}\right)}{\Im(\tau)} .
\end{aligned}
$$

Инстантоны - это классические решения, т.е. гармонические функции на торе с граничными условиями $\left[\epsilon, \epsilon^{\prime}\right]$ :

$$
\varphi_{n+\epsilon, m+\epsilon^{\prime}}^{(I)}(x, t)=2 \pi R\left((n+\epsilon) x+\left(m+\epsilon^{\prime}\right) t\right),
$$

где $(m, n) \in \mathbb{Z}^{2}$. Решение (D.3) - единственное решение с монодромией $(2 \pi(n+\epsilon) R$, $\left.2 \pi\left(m+\epsilon^{\prime}\right) R\right)$. Для краткости записи обозначим $v=2 \pi(n+\epsilon) R$ и $w=2 \pi\left(m+\epsilon^{\prime}\right) R$, так что $\varphi_{I}(x, t)=x v+t w$. Действие для конфигурации (D.3) имеет вид

$$
\begin{aligned}
S\left[\varphi_{n+\epsilon, m+\epsilon^{\prime}}^{(I)}\right] & =\frac{g}{2 \pi} \int_{\mathbf{T}_{\Gamma}}\left(\nabla \varphi_{n+\epsilon, m+\epsilon^{\prime}}^{(I)}\right)^{2}= \\
& =\frac{2 g}{\pi} \int_{D_{\Gamma}}\left(\partial_{z} \varphi_{n+\epsilon, m+\epsilon^{\prime}}^{(I)}\right)\left(\partial_{\bar{z}} \varphi_{n+\epsilon, m+\epsilon^{\prime}}^{(I)}\right) \frac{d \bar{z} \wedge d z}{2 i},
\end{aligned}
$$

где $D_{\Gamma}$ - элементарная ячейка решетки Г. Получим

$$
\begin{aligned}
& \partial_{z} \varphi_{n+\epsilon, m+\epsilon^{\prime}}^{(I)}=\frac{1}{2 i \omega_{1}}\left(i v+\frac{w}{\Im(\tau)}-\frac{\Re(\tau)}{\Im(\tau)} v\right), \\
& \partial_{\bar{z}} \varphi^{(I)}=\frac{1}{2 i \bar{\omega}_{1}}\left(i v-\frac{w}{\Im(\tau)}+\frac{\Re(\tau)}{\Im(\tau)} v\right) .
\end{aligned}
$$

Наконец, действие для инстантона $\left(n+\epsilon, m+\epsilon^{\prime}\right)$ имеет вид

$$
S\left[\varphi_{\epsilon+n, \epsilon^{\prime}+m}^{(I)}\right]=2 \pi R^{2} g \Im(\tau)\left((n+\epsilon)^{2}+\left(\frac{m+\epsilon^{\prime}-\Re(\tau)(n+\epsilon)}{\Im(\tau)}\right)^{2}\right) .
$$

\section{D.2. Вычисление статистической суммы}

Если $\varphi$ и $\varphi_{\epsilon+n, \epsilon^{\prime}+m}^{(I)}$ имеют одинаковые монодромии, то $\phi=\varphi-\varphi_{\epsilon+n, \epsilon^{\prime}+m}^{(I)}-$ нулевую монодромию. Более того, т.к. действие квадратично по полям и достигает экстремума на поле $\varphi^{(I)}$, то

$$
S\left[\varphi_{\epsilon+n, \epsilon^{\prime}+m}^{(I)}+\phi\right]=S[\phi]+S\left[\varphi_{\epsilon+n, \epsilon^{\prime}+m}^{(I)}\right] .
$$

Поэтому статистическая сумма факторизуется:

$$
Z_{\left[\epsilon, \epsilon^{\prime}\right]}(g, R)=Z_{f} \sum_{(m, n) \in \mathbb{Z}^{2}} \exp \left\{-S\left[\varphi_{\epsilon+n, \epsilon^{\prime}+m}^{(I)}\right]\right\},
$$

где $Z_{f}$ содержит вклад флуктуаций при нулевой монодромии, а мы сейчас намерены вычислить инстантонный вклад, который даст добавку к фллуктуационному вкладу, вычисленному в приложении С. 


\section{D.3. Введение калибровочного поля и инстантонный вклад}

Используем следуюшее полезное свойство: пусть $A$ и $B$ - замкнутые 1-формы на торе, тогда

$$
\int_{\mathbf{T}} A \wedge B=\int_{(a)} A \int_{(b)} B-\int_{(b)} A \int_{(a)} B
$$

где $(a)$ и $(b)$ - следующие циклы:

$$
(a): t \in[0,1] \rightarrow t, \quad(b): t \in[0,1] \rightarrow \tau t .
$$

Используя (D.9) и беря голономии (3.2):

$$
\int_{(a)} A=2 \pi a, \quad \int_{(b)} A=2 \pi b
$$

и монодромии (3.15) поля $\varphi$ вдоль $(a)$ - и $(b)$-циклов, мы получим

$$
S[\varphi, A]=\frac{g}{2 \pi} \int(\nabla \varphi)^{2}+4 \pi i\left((\epsilon+n) b-\left(\epsilon^{\prime}+m\right) a\right)
$$

Очевидно, что взаимодействие с калибровочным полем добавляет в действие топологический член.

Вклад инстантонов. Если $f$ - квадратично-интегрируемая функция на $\mathbb{R}$, зададим преобразование Фурье:

$$
(\mathcal{F} \cdot f)(y)=\int_{-\infty}^{+\infty} d x e^{2 i \pi x y} f(x) .
$$

Тогда формула пересуммирования Пуассона примет вид

$$
\sum_{n=-\infty}^{+\infty} f(n)=\sum_{n=-\infty}^{+\infty}(\mathcal{F} \cdot f)(n)
$$

Используя (D.14), мы выведем выражение для суммирования по $m$ в формуле (D.8), и окончательно вклад инстантонов будет иметь вид

$$
\left(\frac{\Im(\tau)}{2 R^{2} g}\right)^{1 / 2} \sum_{(m, n) \in \mathbb{Z}^{2}} e^{-2 i \pi m \epsilon^{\prime}} q^{\frac{1}{2}\left((n+\epsilon) R \sqrt{g}+\frac{m}{2 R \sqrt{g}}\right)^{2}} \bar{q}^{\frac{1}{2}\left((n+\epsilon) R \sqrt{g}-\frac{m}{2 R \sqrt{g}}\right)^{2}}
$$

\section{ЭЛЛИПТИЧЕСКИЕ И ТЕТА-ФУНКЦИИ}

ПРИЛОЖКЕНИЕ Е

В этом приложении мы приводим базисные определения и формулы для эллиптических и тета-функций. Отметив многочисленные работы на эту тему, скажем, что [91, 109] и [62] содержат весь необходимый материал. 


\section{Е.1. Основные определения}

Обозначим через $\tau$ элемент верхней полуплоскости, $q=e^{2 \pi i \tau}$. Пусть $\Gamma=\omega_{1} \mathbb{Z} \oplus$ $\omega_{2} \mathbb{Z}\left(\Im\left(\omega_{2} / \omega_{1}\right)>0\right)$ будет решеткой в $\mathbb{C}$, обозначим модулярный параметр через $\tau=$ $\omega_{2} / \omega_{1}$. Эллиптическая кривая, ассоциированная с такой решеткой, имеет вид тора $\mathbb{T}_{\Gamma}=\mathbb{C} /\left(\omega_{1} \mathbb{Z} \oplus \omega_{2} \mathbb{Z}\right)$. Его комплексная структура параметризована модулярным параметром $\tau$ по модулю действия группы $P S L(2, \mathbb{Z})$.

Функция Вейерштрасса, ассоциированная с решеткой $\Gamma$, определена следуюшим образом:

$$
\wp_{\Gamma}(z)=\frac{1}{z^{2}}+\sum_{\omega \in \Gamma \backslash\{0\}}\left(\frac{1}{(z-\omega)^{2}}-\frac{1}{\omega^{2}}\right) .
$$

Она дважды периодична и задает мероморфную функцию на торе $\mathbb{T}_{\Gamma}$. Интересно рассмотреть предел $\tau \rightarrow+i \infty$ :

$$
\lim _{\tau \rightarrow+i \infty}\left(\wp_{\mathbb{Z} \oplus \tau \mathbb{Z}}(z)\right)=\frac{1}{z^{2}}+\sum_{n \in \mathbb{Z} \backslash\{0\}} \frac{1}{(z-n)^{2}}=\frac{\pi^{2}}{\sin (\pi z)^{2}}
$$

Римановы тета-функции с характеристиками определены следующим образом:

$$
\vartheta\left[\begin{array}{l}
a \\
b
\end{array}\right](z, \tau)=\sum_{n \in \mathbb{Z}+a} q^{n^{2} / 2} e^{2 \pi i n(z+b)} .
$$

При $(a, b) \in\{0,1 / 2\}^{2}$ мы получим функции Якоби. Используя обозначения работы [25, приложение $9 . \mathrm{A}]$, имеем

$$
\begin{aligned}
& \vartheta_{1}(z, \tau)=\vartheta\left[\begin{array}{c}
1 / 2 \\
1 / 2
\end{array}\right](z, \tau), \\
& \vartheta_{2}(z, \tau)=\vartheta\left[\begin{array}{c}
1 / 2 \\
0
\end{array}\right](z, \tau), \\
& \vartheta_{3}(z, \tau)=\vartheta\left[\begin{array}{l}
0 \\
0
\end{array}\right](z, \tau), \\
& \vartheta_{4}(z, \tau)=\vartheta\left[\begin{array}{c}
0 \\
1 / 2
\end{array}\right](z, \tau) .
\end{aligned}
$$

Технически это голоморфные сечения спиновых расслоений над тором $\mathbb{T}_{(\mathbb{Z} \oplus \tau \mathbb{Z})}$, которые возникают в теории киральных фермионов на торе [33].

\section{Е.2. Соотношения между эллиптическими и} тета-функциями и обратный лапласиан на торе

Обратный лапласиан на торе $\mathbb{T}_{\Gamma}$ может быть выражен в терминах $\vartheta_{1}$ [25]:

$$
\Delta^{-1}(z)=\frac{1}{2 \pi} \log \left(\left|\omega_{1} e^{-i \pi \frac{z}{\omega_{1}}} \frac{\vartheta_{1}\left(\frac{z}{\omega_{1}}, \tau\right)}{\vartheta_{1}^{\prime}(0, \tau)}\right|\right)-\frac{1}{2}\left(\Im\left(\frac{z}{\omega_{1}}\right)+\frac{\Im\left(\frac{z}{\omega_{1}}\right)^{2}}{\Im(\tau)}\right)
$$


Обратный лапласиан удовлетворяет следуюшему уравнению Грина:

$$
\Delta\left(\Delta^{-1}\right)=\delta-\frac{1}{\mathcal{A}}
$$

где $\mathcal{A}=\Im\left(\omega_{1} \bar{\omega}_{2}\right)-$ плошадь тора. Связь с ю-функцией Вейерштрасса имеет вид

$$
\begin{aligned}
& \left(\partial_{z}^{2} \Delta^{-1}\right)(z)=\frac{1}{4 \omega_{1}^{2} \Im(\tau)}-\frac{1}{4 \pi} \wp_{\Gamma}(z), \\
& \left(\partial_{\bar{z}}^{2} \Delta^{-1}\right)(z)=\frac{1}{4 \bar{\omega}_{1}^{2} \Im(\tau)}-\frac{1}{4 \pi} \overline{\wp_{\Gamma}(z)} .
\end{aligned}
$$

Чтобы вычислить корреляторы в операторном формализме, нам надо уметь переставлять $\Re(z)$ и $\Im(z)$, которые суть пространственная координата и координата мнимого времени на торе. Таким образом, полезно использовать фурье-разложение для функции Вейерштрасса. Обозначая $x=\exp (2 \pi i z)$, получим при $|q|<|x|<|q|^{-1}[109]$

$$
-\frac{\wp(z)}{4 \pi^{2}}=\frac{1}{12}-2 \sum_{n=1}^{+\infty} \frac{q^{n}}{\left(q^{n}-1\right)^{2}}+\frac{x}{(1-x)^{2}}+\sum_{n=1}^{+\infty} \frac{n q^{n}}{1-q^{n}}\left(x^{n}+x^{-n}\right) .
$$

Функция

$$
\frac{x}{(1-x)^{2}}=\frac{-1}{4 \sin ^{2}(\pi z)}
$$

может быть разложена в ряд по $x$ в каждой из областей $|x|>1$ и $|x|<1$.

Функция Вейерштрасса имеет комплексную первообразную $\xi$-функцию: $\xi^{\prime}=-\wp$. Ее разложение дается формулой

$$
\xi(z)=\frac{1}{z}+\sum_{\omega \in \Gamma \backslash\{0\}}\left(\frac{1}{z-\omega}+\frac{z+\omega}{\omega^{2}}\right) .
$$

Эта функция многозначна на торе. Обозначив через $\eta_{1,2}=\xi\left(z+\omega_{1,2}\right)-\xi(z)$ ее монодромии, получим

$$
\omega_{1} \eta_{2}-\omega_{2} \eta_{1}=2 \pi i
$$

Используя разложение Фурье для $\wp$, мы выразим $\eta_{1}$ как функцию $\omega_{1}$ и $\omega_{2}$ :

$$
\eta_{1}=\frac{4 \pi^{2}}{\omega_{1}}\left(\frac{1}{12}-2 \sum_{n=1}^{+\infty} \frac{q^{n}}{\left(1-q^{n}\right)^{2}}\right)
$$

В подходе функционального интеграла в КТП Люттингера производные $\Delta^{-1}$ являются обобщенными функциями. Для обобшенной функции $T$ и пробной функции $f$ производная $\partial_{\mu} T$ определена следующим образом:

$$
\left(\partial_{\mu} T\right) \cdot f=-T \cdot\left(\partial_{\mu} f\right)
$$

Для точности будем обозначать через [ $\varphi$ ] регулярную обобщенную функцию, задаваемую интегрируемой функцией $\varphi$,

$$
[\varphi] \cdot f=\int \varphi f
$$


На плоскости функция Грина оператора Лапласа имеет вид

$$
\Delta_{\mathbb{C}}^{-1}(z)=\frac{1}{2 \pi} \log (|z|)
$$

Ее первая производная $\partial_{z}\left[\Delta_{\mathbb{C}}^{-1}\right]$ как обобшенная функция имеет форму

$$
\left[\frac{1}{4 \pi z}\right]
$$

Хотя $1 / z^{2}$, будучи неинтегрируемой функцией, не задает регулярной обобщенной функции, тем не менее вторую производную обратного лапласиана $\left[\Delta_{\mathbb{C}}^{-1}\right]$ можно соотнести с отображением $z \mapsto z^{-2}$ через формулу интегрирования по частям. Пусть $K$ - ограниченная область $\mathbb{C}$ с гранишей $\partial K$, тогда

$$
\int_{K}\left(\partial_{z} f\right) g=-\int_{K} f\left(\partial_{z} g\right)+\frac{i}{2} \int_{\partial K}(f g)(z, \bar{z}) d \bar{z} .
$$

Теперь расщепляя интеграл $\int\left(\partial_{z} f\right) / z$ на два вклада $(|z|>\varepsilon$ и $|z|<\varepsilon)$ и применяя вышеприведенные формулы, получим для любой пробной функции $f$

$$
\partial_{z}\left[\frac{1}{z}\right] \cdot f=\lim _{\varepsilon \rightarrow 0^{+}}\left(\int_{|z|>\varepsilon} \frac{f(z, \bar{z})}{z^{2}} d^{2} z\right) .
$$

Эта формула “интеграла по главному значению” устанавливает связь между $\partial_{z}^{2}\left[\Delta_{\mathbb{C}}^{-1}\right]$ и $z \mapsto z^{-2}$. Формула (Е.18) также дает сразу хорошо известное тождество

$$
\partial_{z}\left[\frac{1}{\bar{z}}\right]=\pi \delta
$$

где правая часть появляется из $\partial\{z,|z|>\varepsilon\}$. Эти формулы легко обобшаются на случай тора $\mathbb{C} /(\mathbb{Z} \oplus \tau \mathbb{Z})$. Из периодичности по решетке и анализа сингулярности получим

$$
\partial_{z}\left[\Delta^{-1}\right]=\frac{1}{4 \pi}\left[\frac{\vartheta_{1}^{\prime}(z, \tau)}{\vartheta_{1}(z, \tau)}+2 \pi i \frac{\Im(z)}{\Im(\tau)}\right]=\frac{1}{4 \pi}\left[\xi(z)-\eta_{1} z+2 \pi i \frac{\Im(z)}{\Im(\tau)}\right] .
$$

Дифференцируя еше раз по $z$, получим, что для любой пробной функции $f$ на торе ${ }^{17)}$

$$
\partial_{z}^{2}\left[\Delta^{-1}\right] \cdot f=\lim _{\varepsilon \rightarrow 0^{+}}\left(\int_{|z|>\varepsilon} d^{2} z\left(\frac{1}{4 \Im(\tau)}-\frac{1}{4 \pi} \wp(z)\right) f(z, \bar{z})\right)
$$

где интеграл идет по единичной клетке решетки $\mathbb{Z} \oplus \tau \mathbb{Z}$ с ограничением $|z|>\varepsilon$. Похожим образом найдем, что

$$
\partial_{z} \partial_{\bar{z}}\left[\Delta^{-1}\right]=\frac{1}{4}\left(\delta-\frac{1}{\mathcal{A}}\right)
$$

\footnotetext{
17) Интегрирование идет по фундаментальной области (клетке) решетки $\mathbb{Z} \oplus \tau \mathbb{Z}$, центр которой предполагается находящимся в точке 0.
} 


\section{E.3. Модулярные свойства тета-функций}

Модулярная группа $S L(2, \mathbb{Z})$ порождается двумя матрицами [110, гл. 7$]$ :

$$
t=\left(\begin{array}{ll}
1 & 1 \\
0 & 1
\end{array}\right) \quad \text { и } \quad s=\left(\begin{array}{cc}
0 & -1 \\
1 & 0
\end{array}\right) .
$$

Тогда получим

$$
\begin{aligned}
& \vartheta\left[\begin{array}{l}
a \\
b
\end{array}\right](z+1, \tau+1)=e^{i \pi a(1-a)} \vartheta\left[\begin{array}{c}
a \\
b+a+1 / 2
\end{array}\right](z, \tau), \\
& \vartheta\left[\begin{array}{l}
a \\
b
\end{array}\right]\left(\frac{-z}{\tau},-\frac{1}{\tau}\right)=(-i \tau)^{1 / 2} e^{-2 \pi i z b} e^{i \pi z^{2} / \tau} \vartheta\left[\begin{array}{c}
b \\
-a
\end{array}\right](z, \tau) .
\end{aligned}
$$

$\eta$-Функция Дедекинда, определенная в (C.8), преобразуется так:

$$
\begin{aligned}
& \eta(\tau+1)=e^{i \pi / 12} \eta(\tau), \\
& \eta(-1 / \tau)=(-i \tau)^{1 / 2} \eta(\tau) .
\end{aligned}
$$

Комбинируя функцию Дедекинда и тета-функции Римана, мы получим конечномерные унитарные представления модулярной группы. При $N \geqslant 1$ и $n \in \mathbb{Z} / N \mathbb{Z}$ имеем

$$
\chi_{n}(\tau)=\frac{\vartheta\left[\begin{array}{c}
n / N \\
0
\end{array}\right](0, N \tau)}{\eta(\tau)} .
$$

Тогда получим

$$
\begin{aligned}
& \chi_{n}(\tau+1)=\exp \left\{2 \pi i\left(\frac{n^{2}}{2 N}-\frac{1}{24}\right)\right\} \chi_{n}(\tau), \\
& \chi_{n}(-1 / \tau)=\frac{1}{\sqrt{N}} \sum_{m \in \mathbb{Z} / N \mathbb{Z}} e^{-2 \pi i n m / N} \chi_{m}(\tau) .
\end{aligned}
$$

Это унитарное линейное представление $S L(2, \mathbb{Z})$ появляется во многих конформных теориях поля типа рациональной гауссовой модели [111], активно используемой в данной работе, а также в $S U(N)$-моделях Весса-Зумино-Виттена на уровне 1 [112]. Ядро этого представления содержит ядро конгруэнц-подгруппы $\Gamma(12 N)$ группы $S L(2, \mathbb{Z})$. Напомним, что

$$
\Gamma(N)=\left\{M \in S L(2, \mathbb{Z}), M \equiv\left(\begin{array}{ll}
1 & 0 \\
0 & 1
\end{array}\right)(\bmod N)\right\},
$$

и, таким образом, это представление возникает как представление фактор-групшы $S L(2, \mathbb{Z}) / \Gamma(12 N) \simeq S L(2, \mathbb{Z} / 12 N \mathbb{Z})$.

\section{НЕРЕЛЯТИВИСТСКИЕ ФЕРМИОНЫ}

В этом приложении мы вычислим корреляции плотность-плотность газа нерелятивистских фермионов. Рассмотрим теорию при нулевой температуре, когда важны детали фермионной динамики. Наша цель состоит в том, чтобы прояснить результаты КТП Люттингера, полученные в разделе 5.1 . 


\section{F.1. Отклик на внешний потенциал}

Вычислим линейный отклик плотности заряда на внешний потенциал для нерелятивистских свободных фермионов. Внешний потенциал может рассматриваться как стационарное возмущение. Стандартная стационарная теория возмушений дает поправку первого порядка к собственному значению $\left|\psi_{n}\right\rangle$ :

$$
\left|\psi_{n}^{(1)}\right\rangle=\left|\psi_{n}\right\rangle+\sum_{m \neq n} \frac{V_{m n}}{E_{n}-E_{m}}\left|\psi_{m}\right\rangle,
$$

где $V$ - матрица возмущения. Кроме того, мы получим поправки первого порядка к матричному элементу $A_{m n}=\left\langle\psi_{n}^{(1)}|A| \psi_{m}^{(1)}\right\rangle$ :

$$
\sum_{l \neq m} \frac{V_{l m} A_{n l}}{E_{m}-E_{l}}+\sum_{l \neq n} \frac{V_{n l} A_{l m}}{E_{n}-E_{l}}
$$

Фурье-образ отклика плотности $\rho(q)$ на моду внешнего потенциала $V(q)$ с волновым вектором $q$ может быть тем самым выражен в линейном приближении в терминах плотности состояния возмушения частища-дырка как $\rho(q)=\nu(q) V(q)$, где

$$
\nu(q)=\int_{0}^{+\infty} \frac{d \omega}{\omega} \nu(q, \omega)
$$

Плотность $\nu(q, \omega)$ возмушения частица-дырка имеет вид

$$
\nu(q, \omega)=\sum_{q} n(q)(1-n(q)) \delta(\omega-\epsilon(k)-\epsilon(q+k)) .
$$

Вычисляя это выражение, мы получим явное выражение для отклика плотности на внешний потенциал:

$$
\nu(q)=-\frac{L}{\pi v_{\mathrm{F}}} \frac{1}{2 x} \log \left(\left|\frac{1+x}{1-x}\right|\right), \quad x=\frac{q}{2 k_{\mathrm{F}}} .
$$

Это выражение интересно по двум причинам. Во-первых, в точке $q=2 k_{\mathrm{F}}$ имеется логарифмическая сингулярность (сингулярность Кона), которая свидетельствует о наличие так называемой нестабильности Пайерлса [110]. Эта нестабильность ответственна за появление щели в спектре и за переход энергии в волну плотности заряда, если, например, система взаимодействует с неоднородностями решетки. Электронные взаимодействия также могут приводить к такому переходу. Во-вторых, при малых импульсах мы получим, что $\nu(q) \simeq-L / \pi v_{\mathrm{F}}$, это и есть результат, следующий из $\mathrm{KT \Pi} \mathrm{(5.12).}$ 


\section{F.2. Корреляции плотность-плотность}

Рассмотрим фермионные моды импульсов $2 \pi n / L$. Оператор плотности заряда выражается через операторы рождения-уничтожения следующим образом:

$$
\hat{\rho}(\sigma)=\frac{1}{L} \sum_{k, q} e^{i q \sigma} \hat{c}_{k+q}^{\dagger} \hat{c}_{k}
$$

При нулевой температуре система находится в вакууме Ферми $|F\rangle$, где заняты все фермионные состояния с импульсами $k$ такими, что $|k|<k_{\mathrm{F}}$, а все остальные состояния пусты. В системе имеются $N=2 n_{\mathrm{F}}+1$ частиц, волновой вектор Ферми $k_{\mathrm{F}}=$ $2 \pi\left(n_{\mathrm{F}}+1 / 2\right) / L$ и вводится ультрафиолетовое обрезание: импульсы ограничены по абсолютной величине числом $\pi / a$, где $a$ - шаг решетки. Мы собираемся вычислить одновременную двухточечную функцию

$$
\langle F|\hat{\rho}(\sigma) \hat{\rho}(0)| F\rangle=\frac{1}{L^{2}} \sum_{k, k^{\prime}, q, q^{\prime}} e^{i q^{\prime} \sigma}\left\langle F\left|c_{k^{\prime}+q^{\prime}}^{+} c_{k^{\prime}} c_{k+q}^{+} c_{k}\right| F\right\rangle
$$

и будем различать два случая: $q=0$ и $q \neq 0$.

F.2.1. Случай $q=0$. Неисчезающие вклады будут наблюдаться при $\left|k^{\prime}\right|<k_{\mathrm{F}}$ и $q^{\prime}=0$. Вклад в коррелятор (F.7) равен $(N / L)^{2}=\left(k_{\mathrm{F}} / \pi\right)^{2}$, т.е. квадрату среднего от величины $\rho(\sigma)$. При переходе к нормальным произведениям этот вклад исчезает и поэтому несушествен для нашего обсуждения.

F.2.2. Случай $q \neq 0$. Теперь $|k+q|>k_{\mathrm{F}}$, и мы получим $k^{\prime}=k+q$ и $q^{\prime}=-q$, иначе матричный элемент в (F.7) обрашается в нуль. Прямое вычисление различных сумм дает явное выражение для связных корреляций плотность-плотность:

$$
\begin{aligned}
\langle F|\hat{\rho}(\sigma) \hat{\rho}(0)| F\rangle_{c}= & \frac{2 k_{\mathrm{F}}}{\pi L} \frac{\cos \left(\left(\frac{\pi}{2 a}+k_{\mathrm{F}}-\frac{\pi}{L}\right) \sigma\right) \sin \left(\left(\frac{\pi}{2 a}-k_{\mathrm{F}}\right) \sigma\right)}{\sin \left(\frac{\pi \sigma}{L}\right)}+ \\
& +\frac{k_{\mathrm{F}}}{\pi L} \frac{\sin \left(\left(2 k_{\mathrm{F}}-\frac{\pi}{L}\right) \sigma\right)}{\sin \left(\frac{\pi \sigma}{L}\right)}-\frac{1}{L^{2}} \frac{\sin ^{2}\left(k_{\mathrm{F}} \sigma\right)}{\sin ^{2}\left(\frac{\pi \sigma}{L}\right)} .
\end{aligned}
$$

Первый член возникает из вклада $|q| \geqslant 2 k_{\mathrm{F}}$ (все $k$-матричные элементы отличны от нуля, если $k$ лежит внутри моря Ферми), а два последних члена - из вклада $|q|<2 k_{\mathrm{F}}$ (в этом случае только некоторые из матричных элементов отличны от нуля).

Чтобы восстановить результаты КТП, надо взять термодинамический предел и рассмотреть корреляции только при больших расстояниях: $1 / k_{\mathrm{F}} \ll \sigma \ll L$ и $1 / a \ll \sigma \ll L$, причем $k_{\mathrm{F}}$ и $a$ фиксированы. Связная корреляционная функция плотность-плотность в этом пределе имеет вид

$$
\langle F|\hat{\rho}(\sigma) \hat{\rho}(0)| F\rangle_{c} \sim \frac{2 k_{\mathrm{F}}}{\pi} \delta(\sigma)-\frac{1}{2 L^{2}} \sin ^{-2}\left(\frac{\pi \sigma}{L}\right) .
$$

Для вычисления вклада $\delta(\sigma)$ потребуется следуюшее выражение:

$$
\frac{1}{L} \frac{\sin \left(\frac{\pi \lambda \sigma}{L}\right)}{\sin \left(\frac{\pi \sigma}{L}\right)} \longrightarrow \delta(\sigma)
$$


выполненное в пределе $\lambda \rightarrow+\infty$, где $\lambda$ выбирается нечетным иельмм числом. Другой вклад в (F.9) получается взятием среднего по шкале длины $l$ такой, что $1 / k_{\mathrm{F}} \ll l \ll \sigma$ : $\sin ^{2}\left(k_{\mathrm{F}} \sigma\right)$ заменяется на $1 / 2$.

Ответы из КТП для этой корреляционной функции даются уравнениями (5.4) и (5.5). В отсутствие внешнего потенциала КТП имеем

$$
\langle\rho(\sigma, t) \rho(0,0)\rangle_{c}=\frac{-1}{2 \pi^{2} \alpha} \Re\left(\wp\left(\sigma+i v_{S} t\right)\right)+\frac{1}{2 \pi \alpha} \delta(\sigma) \delta(t) .
$$

В пределе нулевой температуры мы используем асимптотическое поведение (Е.2) ю-функции Вейерштрасса в пределе $\tau \rightarrow+i \infty$, откуда получим одновременной коррелятор при нулевой температуре:

$$
-\frac{1}{2 \alpha L^{2}} \sin ^{-2}\left(\pi \frac{\sigma}{L}\right)+\frac{1}{2 \pi \alpha} \delta(\sigma) \delta(0) .
$$

Второй член плохо определен: вклад $\delta(0)$ появляется из условия (Е.2) при $t=0$. В действительности эта обобшенная дельта-функция должна быть регуляризована. Детальное вычисление в задаче нерелятивистских фермионов показывает, что эта расходимость размазьвается по конечной области длины порядка $k_{\mathrm{F}}^{-1}$. Первый член в (F.12)это среднее от нерелятивистского коррелятора по области длины, гораздо большей чем микроскопический масштаб длины $k_{\mathrm{F}}^{-1}$, что и завершает отождествление результатов КТП в пределе отсутствия взаимодействий с результатами теории нерелятивистских свободных фермионов.

\section{ПРИЛОЖЕНИЕ G \\ ОПЕРАТОРНЫЕ ВЫЧИСЛЕНИЯ И СРАВНЕНИЕ С РЕЗУЛЬТАТАМИ КТП}

В этом приложении мы получим некоторые из результатов КТП в терминах бозонизации киральных мод. В отличие от приложения $\mathrm{F}$, мы сразу обсуждаем релятивистские фермионы, но при вычислении в теории со взаимодействием будет использовано преобразование Боголюбова (2.16) и (2.17). Например, в этом киральном подходе мы рассмотрим корреляции плотность-плотность. Более точно, мы вычислим производяший функционал для одновременных корреляторов плотности, в результате чего будет воспроизведен результат КТП.

После введения внешнего потенциала, линейно присоединенного к плотности, или эквивалентно поля источника для корреляций плотности задача сводится к вычислению бозонного гамильтониана обшего вида $h=\omega a^{+} a+\lambda\left(a^{+}+a\right)$, где второй член отвечает за спаривание с потенциалом. Этот гамильтониан затем диагонализуется унитарным преобразованием. Соответствующий унитарньй оператор $U[V]$ имеет вид

$$
U[V(\sigma)]=\exp \left(\frac{i}{2 v_{S} \sqrt{\alpha}} \int_{0}^{L} d \sigma \eta(\sigma)(J(\sigma)+\bar{J}(\sigma))\right),
$$

где $\eta^{\prime}(\sigma)=\mathcal{V}(\sigma)$. Он действует на $U(1)$-токи по формуле

$$
\begin{aligned}
& J^{\prime}(\sigma)=U[V(\sigma)] J(\sigma) U[V(\sigma)]^{-1}=J(\sigma)+\frac{\mathcal{V}(\sigma)}{2 \pi v_{S} \sqrt{\alpha}} \\
& \bar{J}^{\prime}(\sigma)=U[V(\sigma)] \bar{J}(\sigma) U[V(\sigma)]^{-1}=\bar{J}(\sigma)+\frac{\mathcal{V}(\sigma)}{2 \pi v_{S} \sqrt{\alpha}}
\end{aligned}
$$


Эти преобразованные операторы удовлетворяют $\widehat{U(1)}$-коммутационным соотношениям. Полный гамильтониан (включаюший кинетический член, фермион-фермионные взаимодействия и спаривание плотности с внешним потенциалом) преобразуется следуюшим образом при унитарном преобразовании (G.1):

$$
H[V(\sigma), J, \bar{J}]=H\left[0, J^{\prime}, \bar{J}^{\prime}\right]+\frac{1}{2 \pi \alpha v_{S}} \int_{0}^{L} \mathcal{V}(\sigma)^{2} d \sigma,
$$

где мы использовали формулу (2.20), в которой приведены свободный член и член фермион-фермионного взаимодействия. Мы также находим

$$
\int_{0}^{L} b(\sigma) \rho_{[J, \bar{J}]}(\sigma)=\int_{0}^{L} b(\sigma) \rho_{\left[J^{\prime}, \bar{J}^{\prime}\right]}(\sigma)-\frac{1}{\pi \alpha v_{S}} \int_{0}^{L} \mathcal{V}(\sigma) b(\sigma) d \sigma .
$$

Таким образом, используя уравнения (G.2)-(G.5) и цикличность следа, мы получим выражение для производящего функционала (5.1)

$$
W_{[V(\sigma), \chi, q]}^{(0)}[b(\sigma)]=W_{[0, \chi, q]}^{(0)}[b(\sigma)] \exp \left\{-\frac{1}{\pi \alpha} \int_{0}^{L} b(\sigma) \mathcal{V}(\sigma) d \sigma\right\} .
$$

Среднее $b_{0}$ поля $b$ спаривается с полньм зарядом и дает следующий вклад в линеаризованный член (5.3):

$$
\int_{0}^{L} \rho(\sigma) b_{0} d \sigma=\frac{q}{L} \int_{0}^{L} b(\sigma) d \sigma
$$

Таким образом, мы восстановили линейный член, отвечающий (5.3). Член

$$
\frac{1}{2 \pi \alpha v_{S}} \int \mathcal{V}(\sigma)^{2}
$$

которьй появляется в правой части уравнения (G.4), соответствует аномалии киральных калибровочных преобразований с точностью до множителя $v_{S}$, который был положен равным единице в (3.13).

Квадратичный вклад в производяший функционал вычисляется, если $\exp \left\{\int b(\sigma) \times\right.$ $\rho(\sigma) d \sigma\}$ имеет вид $e^{A+A^{\dagger}}$, где величина $\left[A, A^{\dagger}\right]$ пропорциональна единице, более точно ${ }^{18)}$,

$$
A=\frac{1}{\sqrt{\alpha}} \sum_{n=1}^{+\infty}\left(b_{-n} J_{n}+b_{n} \bar{J}_{n}\right),
$$

и искомый коммутатор имеет вид

$$
\left[A, A^{\dagger}\right]=\frac{2}{\alpha} \sum_{n=1}^{+\infty} n\left|b_{n}\right|^{2}
$$

\footnotetext{
18) Здесь среднее от $b$ по окружности равно нулю.
} 
Формула Глаубера позволяет вычислить среднее при конечной температуре от $\left\langle e^{A^{\dagger}} e^{A}\right\rangle$. Однако для отдельного гармонического осциллятора выполняется следующее тождество между средними при конечной температуре:

$$
\left\langle e^{\lambda a^{\dagger}} e^{\mu a}\right\rangle=e^{\lambda \mu\left\langle a^{\dagger} a\right\rangle}
$$

В нашем случае это дает

$$
W_{[0, \chi, q]}^{(0)}[b(\sigma)]=\exp \left(\frac{1}{\alpha} \sum_{n=1}^{+\infty} n b_{n} b_{-n} \frac{1+q^{n}}{1-q^{n}}\right),
$$

где $q=\exp \left(-2 \pi v_{S} / L\right)$. В этом выражении легко узнать фурье-разложение функции Вейерштрасса. Более точно, формально мы получаем

$$
\lim _{\varepsilon \rightarrow 0^{+}}\left(\frac{\wp(\sigma+i \varepsilon)+\wp(\sigma-i \varepsilon)}{2}\right)=-\eta_{1}+2 \pi^{2} \sum_{n=1}^{+\infty} n \frac{1+q^{n}}{1-q^{n}}\left(x^{n}+x^{-n}\right),
$$

где $\eta_{1}$ обозначает монодромии $\xi$, как и в приложении Е. Однако, поскольку постоянная составляюшая поля $b$ не была учтена в уравнении (G.9), получаются уравнения (5.4) и $(5.5)$.

\section{ДУАЛЬНОСТЬ ДЛЯ СВОБОДНОГО БОЗОНА}

Явное выражение для статистической суммы свободного бозона на торе показывает, что теории при $\alpha=g R^{2}$ и $\alpha^{-1} / 4$ имеют совпадающие статистические суммы. Эта симметрия в конформной теории называется дуальностью [27]. Мы проводим анализ взаимоотношений меж ду этой дуальностью и граничными условиями, наложенными на бозонное поле, а также рассматриваем производящий функционал, которьй соответствует безмассовой модели Тирринга, взаимодействующей с калибровочным полем, как описано в разделе 3 . Наш метод основан на явных преобразованиях функциональных интегралов. В этих вычислениях важно учитьвать нормировочное предписание.

\section{Н.1. Введение вспомогательных полей}

Введем векторное поле такое, что

$$
\exp \left(-\frac{g}{2 \pi} \int\left(\partial_{\mu} \varphi\right)^{2}\right)=\int \mathcal{D}\left[b_{\mu}\right] \exp \left(-\frac{\pi}{2 g} \int b_{\mu} b^{\mu}+i \int b_{\mu} \epsilon^{\mu \nu} \partial_{\nu} \varphi\right)
$$

Затем проинтегрируем по $\varphi$. Получится эффективное действие для поля $b_{\mu}$. Более точно, рассмотрим интеграл

$$
Z_{\mathcal{C}}[A]=\int_{\mathcal{C}} \mathcal{D}_{R, g}[\varphi] \int \mathcal{D}\left[b_{\mu}\right] \exp \left(-\frac{\pi}{2 g} \int\left(b-\frac{A}{\pi R}\right)^{2}+i \int b \wedge d \varphi\right) .
$$


Разложим теперь поле $\varphi$ на классическое решение $\varphi_{c}$, имеющее требуемую монодромию, постоянную составляющую (нулевая мода) и флуктуирующую часть $\xi: \varphi=\varphi_{c}+$ $\varphi_{0}+\xi$. Интегрирование по частям дает

$$
\int b \wedge d \varphi=\int b \wedge d \varphi_{c}+\int \xi d b
$$

Интегрирование по $\xi$ показывает, что $b$ - плоская связность. Тогда мы можем ее разложить как $b=h+d \alpha$, где $h$ - постоянная 1-форма на торе и $\alpha-$ нулевая форма, из которой возникает член, соответствующий дуальному бозонному полю. Ниже эта идея будет выражена более явно.

\section{Н.2. Компактификация дуального поля}

Из формулы (D.9) следует равенство нулю интеграла от формы $d \alpha \wedge d \varphi_{c}$, поскольку форма $d \alpha$ точна. Таким образом, если $b$ замкнута, то интеграл

$$
\int b \wedge d \varphi_{c}
$$

может быть выражен в терминах монодромии $\varphi_{c}$ и голономий $b$ или эквивалентно через голономии формы $h$. Обозначим через $h_{(a, b)}$ эти голономии соответственно вдоль циклов $(a)$ и $(b)$. Введем также величины $n_{(a, b)}$ такие, что монодромии $\varphi_{c}$ выражаются как $2 \pi R n_{(a, b)} \cdot$ Тогда получим

$$
\int b \wedge d \varphi_{c}=2 \pi R\left(h_{(a)} n_{(b)}-h_{(b)} n_{(a)}\right)
$$

Суммируя по дискретному набору значений $n_{(a, b)}$, мы дискретизуем величины $h_{(b, a)}$ :

$$
\sum_{n \in \mathbb{Z}} \exp (2 \pi i R h n)=\frac{1}{R} \sum_{m \in \mathbb{Z}} \delta\left(h-\frac{m}{R}\right) .
$$

Это является причиной, по которой в дуальной теории также возникает компактифищированное дискретизованное поле.

Исследуем теперь меры интегрирования. Определения даны в приложении С. Предположим, что $A$ - постоянное калибровочное поле. Случай наиболее общего калибровочного поля будет рассмотрен в разделе Н.4.

Мы будем рассматривать модулярно-инвариантную статистическую сумму

$$
\begin{aligned}
Z[A]= & 2 \pi R \sum_{(n, m) \in \mathbb{Z}^{2}} \int \mathcal{D}[b] \exp \left\{-\frac{\pi}{2 g} \int b^{2}\right\} \times \\
& \times \int \mathcal{D}_{\perp, g}[\xi] \exp \left(i \int\left(b+\frac{A}{\pi R}\right) \wedge d\left(\varphi_{n, m}+\xi\right)\right)= \\
= & 2 \pi \int \mathcal{D}[b] \exp \left\{-\frac{\pi}{2 g} \int\left(b-\frac{A}{\pi R}\right)^{2}\right\} W_{\text {inst }}[b] \times \\
& \times \int \mathcal{D}_{\perp, g}[\xi] \exp \left\{i \int b \wedge d \xi\right\} .
\end{aligned}
$$


Функционал $W_{\text {inst }}[b]$ возникает из суммы по инстантонам. Рассмотрим разложение $b=$ $h+\tilde{b}$, где постоянная 1-форма $h$ есть форма $b$, усредненная по тору. Мера интегрирования по вспомогательным полям факторизуется: $\mathcal{D}[b]=d^{2} h \mathcal{D}_{\perp}[\tilde{b}]$. Тогда мы получаем

$$
W_{\mathrm{inst}}[h]=\frac{1}{R^{2}} \sum_{\left(m_{a}, m_{b}\right) \in \mathbb{Z}^{2}} \delta\left(\int_{(a)} h-\frac{m_{a}}{R}\right) \delta\left(\int_{(b)} h-\frac{m_{b}}{R}\right) .
$$

Таким образом, обозначая через $h_{\left(l_{a}, l_{b}\right)}$ постоянную 1 -форму голономий $\left(l_{a}, l_{b}\right)$, мы имеeм

$$
\int d^{2} h W_{\mathrm{inst}}[h] \Phi[h]=\frac{1}{\mathcal{A} R^{2}} \sum_{\left(m_{a}, m_{b}\right) \in \mathbb{Z}^{2}} \Phi\left[h_{R^{-1}\left(m_{a}, m_{b}\right)}\right] .
$$

Теперь вычислим интегралы по $\tilde{b}$ и $\xi$. Пусть

$$
F[A, h]=\int \mathcal{D}_{\perp}[\tilde{b}] \int \mathcal{D}_{\perp, g}[\xi] \exp \left\{i \int \tilde{b} \wedge d \xi\right\} \exp \left(-\frac{\pi}{2 g} \int\left(h-\frac{A}{\pi R}+\tilde{b}\right)^{2}\right) .
$$

Интегрируя по $\tilde{b}$, учитывая нормировочное условие для $b$ и используя то, что поле $A$ постоянно, мы получаем

$$
F[A, h]=\frac{\mathcal{A}}{2 g} \int \mathcal{D}_{\perp, g}[\xi] \exp \left(-\frac{g}{2 \pi} \int(d \xi)^{2}-\frac{\pi}{2 g} \int\left(h-\frac{A}{\pi R}\right)^{2}\right) .
$$

После замены переменных $\tilde{\chi}=g \xi$, используя формулу (С.3) для якобиана, находим

$$
F[A, h]=\frac{\mathcal{A}}{2} \int \mathcal{D}_{\perp, g^{-1}}[\widetilde{\chi}] \exp \left(-\frac{1}{2 \pi g} \int\left(d \widetilde{\chi}+\pi h-\frac{A}{R}\right)^{2}\right) .
$$

Собирая все части вместе, получим окончательный ответ:

$$
Z[A]=\int \mathcal{D}_{\frac{1}{2 R}, \frac{1}{g}}[\chi] \exp \left(-\frac{1}{2 \pi g} \int\left(d \chi-\frac{A}{R}\right)^{2}\right),
$$

где поле $\chi$ компактифицировано на окружность радиуса $R^{\prime}=1 / 2 R$. Мы найдем новый параметр $\alpha^{\prime}=R^{\prime 2} g^{-1}=1 / 4 \alpha$, как и следовало ожидать.

\section{Н.3. Дуальность для КТП Люттингера}

Проделаем вычисление в КТП Люттингера. Здесь граничные условия для функшионального интеграла задаются уравнениями (9.2). Вьполним сначала суммирование по монодромиям при фиксированном $\left(\varepsilon, \varepsilon^{\prime}\right)$. Голономии $h_{(a, b)}$ должны быть при этом целыми в единицах $1 / R$. Затем просуммируем по $\left(\varepsilon, \varepsilon^{\prime}\right)$ и получим следуюший ответ:

$$
\begin{aligned}
Z_{\text {Lutt }}^{(r)}[\alpha, A]= & \sum_{\left(u_{a}, u_{b}\right) \in\{0,1\}^{2}}(-1)^{u_{a} u_{b}} e^{i \pi r u_{b}} \times \\
& \times \int_{\left(\pi R^{-1} u_{a}, \pi R^{-1} u_{b}\right)} \mathcal{D}_{\frac{1}{2 R}, \frac{1}{g}}[\chi] \exp \left\{-\frac{1}{2 \pi g} \int\left(\partial_{\mu} \chi-\frac{A}{R}\right)^{2}\right\}
\end{aligned}
$$


Затем переходя к полю, компактифицированному на окружность радиуса $1 / R$, мы восстановим множитель $1 / 2$, возникающий из меры интегрирования по нулевой моде. Из разложения экспоненты получим

$$
Z_{\text {Lutt }}^{(r)}[\alpha, A]=Z_{\text {Lutt }}^{(0)}\left[\alpha^{-1}, \frac{i A^{*}}{\alpha}+b_{r}\right] \exp \left(\frac{1}{2 \pi \alpha} \int A^{2}\right),
$$

где $b_{r}$ отвечает магнитному потоку поля $r \Phi_{0} / 2$. Это следует из формулы

$$
e^{i \pi r u_{b}}=\exp \left\{\frac{i}{\pi R^{-1}} \int b_{r} \wedge d \chi\right\},
$$

где

$$
b_{r}=\pi \frac{r}{L} d \sigma .
$$

Интересно отметить, что дуальность все еше наблюдается, но $\alpha^{\prime}=\alpha^{-1}$ вместо $4 / \alpha$. В отличие от модулярно-инвариантного случая, свободная теория Дирака находится в самодуальной точке ${ }^{19)}$. Заметим также, что электрическое и магнитное поля поменялись местами. Наличие заряда в системе отвечает магнитному потоку в дуальной теории. Таким образом, хотя статистическая сумма в секторе $r=0$ в отсутствие внешнего поля инвариантна при замене $\alpha \mapsto 1 / \alpha$, физика модели меняется.

\section{Н.4. Тождества Уорда в дуальной теории}

Таким образом, мы получили формулы дуальности для компактифицированного бозона и теории Люттингера в ее бозонной формулировке, взаимодействующей с постоянным калибровочным полем. Распространим эти формулы на произвольное калибровочное поле. Напомним, что исходная теория калибровочно-инвариантна относительно преобразований

$$
\begin{aligned}
& A \mapsto A+d \beta, \\
& \varphi \mapsto \varphi .
\end{aligned}
$$

Однако тогда дуальная теория также калибровочно-инвариантна относительно преобразований

$$
\begin{gathered}
A \mapsto A+d \beta, \\
\chi \mapsto \chi+\pi \beta .
\end{gathered}
$$

В дуальной теории киральные калибровочные преобразования отвечают замене

$$
\begin{aligned}
& A \mapsto A+d^{*} \beta, \\
& \chi \mapsto \chi,
\end{aligned}
$$

откуда в дуальной теории $\varphi$ заменяется на $\varphi+i \beta / g R$. Очевидно, что при киральных калибровочных преобразованиях как исходная, так и дуальная теории обладают одинаковыми трансформационными свойствами (3.13). Они также инвариантны при обычных калибровочных преобразованиях. Таким образом, используя теорему Ходжа, мы можем обобщить формулы (Н.9) и (Н.11) на случай произвольного векторного потенциала $A$.

19) Это небольшое отличие от обычного модулярно-инвариантного случая было, на самом деле, замечено Классеном и др. [37]. 


\section{Список литературы}

[1] Л. Д. Ландау. ЖЭТФ. 1957. Т. 32. № 1. С. 59.

[2] Л. Д. Ландау. ЖЭТФ. 1959. Т. 35. № 1. С. 97.

[3] P. W. Anderson. Basic Notions of Condensed Matter Physics. Frontiers in Physics. London: Benjamin, 1984.

[4] А. А. Абрикосов, Л. П. Горьков, И. Е. Дзялоиинский. Методы квантовой теории поля в статистической физике. М.: Наука, 1963.

[5] P. Nozières, J. M. Luttinger. Phys. Rev. 1962. V. 127. P. 1423.

[6] P. Nozières, J. M. Luttinger. Phys. Rev. 1962. V. 127. P. 1431.

[7] И. Е. Дзялошинский. ЖЖЭТФ. 1974. Т. 65. № 1. С. 411.

[8] F.D. M. Haldane. J. Phys. C. Solid State Phys. 1981. V. 14. P. 2585.

[9] P. W. Anderson. Phys. Rev. Lett. 1967. V. 18. P. 1049.

[10] A. Luther, I. Peschel. Phys. Rev. Lett. B. 1974. V. 9. P. 2911.

[11] A. Luther, V. J. Emery. Phys. Rev. Lett. 1974. V. 33. P. 589.

[12] F. D. M Haldane. J. Phys. C. 1979. V. 12.

[13] J. Voit. Phys. Rev. B. 1993. V. 47. P. 6740.

[14] H. J. Schulz. Int. J. Mod. Phys. B. 1991. V. 5. P. 57.

[15] S. Tomonaga. Progr. Theor. Phys. 1950. V. 5. P. 544.

[16] J. M. Luttinger. J. Math. Phys. 1963. V. 4. P. 1154.

[17] D. C. Mattis, E. H. Lieb. J. Math. Phys. 1965. V. 6. P. 304

[18] F.D. M. Haldane. Phys. Rev. Lett. 1980. V. 45. P. 1358.

[19] H. J. Schulz. Interacting fermions in one dimension: from weak to strong correlation. In: Lecture notes at the Jerusalem winter school on theoretical physics, Dec. 1991-Jan. 1992. cond-mat/9302006.

[20] A. A. Belavin, A. B. Polyakov, A. B. Zamolodchikov. Nucl. Phys. B. 1984. V. 241. P. 333.

[21] Р. П. Фейнман, А. Хиббс. Квантовая механика и интегралы по путям. М.: Мир, 1968.

[22] J. Cardy. Nucl. Phys. B. 1986. V. 270. P. 186

[23] J. Cardy. Conformal invariance and statistical mechanics. In: Fields, Strings and Critical phenomena (XLIX Les Houches Session). Ed. E. Brézin, J. Zinn-Justin. Amsterdam-New York: North-Holland, 1990. P. 169.

[24] P. Ginsparg. Applied conformal field theory. In: Fields, Strings and Critical phenomena, (XLIX Les Houches session). Ed. E. Brézin, J. Zinn-Justin. Amsterdam-New York: North-Holland, 1990. P. 1.

[25] C. Itzykson and J. M. Drouffe. Statistical Field Theory. Cambridge: Cambridge Univ. Press, 1989.

[26] S. V. Ketov. Conformal field theory. Singapore: World Scientific, 1995.

[27] R. Dijkgraaf, E. Verlinde, H. Verlinde. Commun. Math. Phys. 1987. V. 115. P. 649.

[28] J. Solyom. Adv. Phys. 1979. V. 28. P. 201.

[29] P. W. Anderson. J. Phys. C. 1970. V. 3. P. 2436.

[30] R. Shankar. Rev. Mod. Phys. 1994. V. 66. P. 129

[31] I. Affleck. Field theory methods and quantum critical phenomena. In: Fields, Strings, and Critical Phenomena (XLIX Les Houches Session). Ed. E. Brézin, J. Zinn-Justin. Amsterdam-New York: North-Holland, 1988. P. 565.

[32] L. Alvarez-Gaume, G. Moore, C. Vafa. Commun. Math. Phys. 1986. V. 106. P. 1.

[33] L. Alvarez-Gaume, J. B. Bost, G. Moore, C. Vafa. Commun. Math. Phys. 1987. V. 112. P. 503.

[34] Ph. Di Francesco, H. Saleur, J. B. Zuber. Nucl. Phys. B. 1987. V. 290. P. 527.

[35] Y.-S. Wu, Y. Yu. Phys. Rev. Lett. 1995. V. 75. P. 890.

[36] F.D. M. Haldane. Phys. Rev. Lett. 1991. V. 67. P. 937.

[37] Th. R. Klassen, E. Melzer. Int. J. Mod. Phys. A. 1993. V. 8. P. 4131.

[38] K. Gawedzki, E. Charpentier. J. Math. Phys. 1993. V. 34. P. 381.

[39] T. Jolicoeur, J. C. Le Guillou. Int. J. Mod. Phys. A. 1993. V. 8. P. 1923.

[40] S. Coleman. Phys. Rev. D. 1975. V. 11. P. 2088. 
[41] S. Mandelstam. Phys. Rev. D. 1975. V. 11. P. 3026.

[42] S. Qin, M. Fabrizio, L. Yu, M. Oshikawa, I. Affleck. Impurity in a Luttinger liquid away from half-filling: A numerical study. cond-mat/9705269.

[43] R. Mélin, B. Douçot, P. Butaud. J. Phys. 1995. V. 4. P. 737.

[44] T. Giamarchi, H. J. Schulz. Phys. Rev. B. 1988. V. 37. P. 325.

[45] X. G. Wen. Phys. Rev. B. 1990. V. 41. P. 12838.

[46] X. G. Wen. Int. J. Mod. Phys. B. 1992. V. 6. P. 1711.

[47] F. D. M. Haldane, E. H. Rezayi. Phys. Rev. B. 1994. V. 50. P. 17199.

[48] B. I. Halperin. Phys. Rev. B. 1982. V. 25. P. 2185.

[49] A. Cappelli, G. V. Dunne, C.A. Truegenberger, G. R. Zemba. Nucl. Phys. B. 1993. V. 398. P. 531.

[50] R. B. Laughlin. Phys. Rev. B. 1982. V. 23. P. 5632

[51] D. Friedan, S. Shenker. Nucl. Phys. B. 1987. V. 281. P. 509.

[52] A. Cappelli, G. R. Zemba. Modular invariant partition functions in the quantum Hall effect. hep-th/9605127.

[53] M. Stone, M. P. A. Fisher. Int. J. Mod. Phys. B. 1994. V. 8. P. 2539.

[54] E. Fradkin. Field theories of condensed matter systems. In: Frontiers in Physics. V. 82. Reading: Addison Wesley, 1991.

[55] В. Г. Кац. Бесконечномерные алгебры Ли. М.: Мир, 1993.

[56] J. Scherk. Rev. Mod. Phys. 1975. V. 47. P. 429.

[57] К. Ициксон, Ж.-Б. Зюбер. Квантовая теория поля. Т. 1,2. М.: Мир, 1984.

[58] W.E. Thirring. Ann. Phys. 1958. V. 3. P. 91.

[59] B. Klaiber. The Thirring model. Talk presented at the Theoretical Physics Institute. Colorado: Univ. of Colorado, 1967.

[60] V. Glaser. Nuovo Cimento. 1958. V. 9. P. 2812

[61] P. Cartier. An Introduction to Zeta Functions. From Number Theory to Physics. (Les Houches, 1989). Berlin-Heidelberg: Springer-Verlag, 1992.

[62] Е. Т. Уиттекер, Дж. Н. Ватсон. Курс современного анализа. Изд. 2-е, в двух частях. М.: Физматгиз, 1962 (часть 1), 1963 (часть 2).

[63] I. Sachs, A. Wipf. Ann. Phys. 1996. V. 249. P. 380.

[64] V. E. Korepin. Commun. Math. Phys. 1980. V. 76. P. 165.

[65] J. Schwinger. Phys. Rev. 1962. V. 128. P. 2425.

[66] В. Л. Березинский. ЖЭТФ. 1973. Т. 65. № 3. С. 1251.

[67] А. А. Гоголин, В. И. Мельников, Е.И. Рашба. ЖЭТФ. 1975. Т. 69. № 1. С. 327.

[68] L. P. Gorkov, O. N. Dorokhov. Solid State Commun. 1976. V. 20. P. 789.

[69] А.А. Овчинников, Н. С. Эрикман. ЖЭТФ. 1977. Т. 73. № 2. С. 650.

[70] А. А. Гоголин, В. И. Мельников. ЖЭТФ. 1977. Т. 73. № 2. С. 706.

[71] В. Л. Березинский, Л. П. Горьков. ЖЭТФ. 1979. Т. 77. № 6. С. 2498.

[72] Т. Н. Анцыгина, Л. А. Пастур, В. А. Слюсарев. ФНТ. 1981. Т. 7. № 1. С. 5.

[73] Л. П. Горьков, О. Н. Дорохов, Ф. В. Пригара. ЖЭЭТ. 1983. Т. 85. № 4. С. 1470.

[74] D. Bernard. Perturbed conformal field theory applied to $2 D$ disordered models: an introduction, low dimensional applications of quantum field theory (Cargese), 1995. hep-th/9509137.

[75] D. Bernard. Nucl. Phys. B. 1995. V. 441. P. 471.

[76] V. G. Knizhnik, A. B. Zamolodchikov. Nucl. Phys. B. 1984. V. 247. P. 83.

[77] L. P. Levy, G. Dolan, J. Dunsmuir, H. Bouchiat. Phys. Rev. Lett. 1990. V. 64. P. 2074.

[78] D. Mailly, C. Chapelier, A. Benoît. Phys. Rev. Lett. 1993. V. 70. P. 2020.

[79] J.-B. Bost. Introduction to compact Riemann surfaces, Jacobians and Abelian varieties, number theory and physics (Les Houches, 1989). Berlin-Heidelberg: Springer-Verlag, 1992.

[80] R. Tao, Y.-S. Wu. Phys. Rev. B. 1984. V. 30. P. 1097.

[81] C. L. Kane, M.P. A. Fisher. Phys. Rev. Lett. 1994. V. 72. P. 724.

[82] M.P. A. Fisher, L. I. Glazman. Transport in a one-dimensional Luttinger liquid. In: Mesoscopic electron transport. Ed. L. Kowenhoven, G. Schoen, L. Sohn. Bruxelles: NATO ASI Series, 1997.

[83] P. Fendley, A.W. W. Ludwig, H. Saleur. Phys. Rev. Lett. 1995. V. 74. P. 3005. 
[84] P. Fendley, A.W.W. Ludwig, H. Saleur. Phys. Rev. Lett. 1995. V. 75. P. 4492.

[85] P. Fendley, A.W.W. Ludwig, H. Saleur. Phys. Rev. B. 1995. V. 52. P. 8934.

[86] P. Fendley, H. Saleur. Non-equilibrium DC noise in a Luttinger liquid with an impurity. cond-mat/9601117.

[87] F. Lesage, S. Saleur, S. Skorik. Nucl. Phys. B. 1996. V. 474. P. 602.

[88] F. A. Smirnov. Quasi-classical study of form factors in finite volume. hep-th/9802132.

[89] L. Saminadayar, D. C. Glattli, Y. Jin, B. Etienne. Phys. Rev. Lett. 1997. V. 79. P. 2526.

[90] G. Moore and N. Seiberg. Lectures on RCFT. In: Physics, Geometry, and Topology. Proc. of the Trieste Spring School, 1988. Singapore: World Scientific, 1990.

[91] ЖК.-П. Серр. Курс арифметики. М.: Мир, 1972.

[92] Н. Коблич. Введение в эллиптические кривые и модулярные формы. М.: Мир, 1988.

[93] P. Degiovanni. Commun. Math. Phys. 1990. V. 127. P. 71.

[94] T. Gannon. Nucl. Phys. B. 1996. V. 491. P. 659.

[95] F. D. M. Haldane, E. H. Rezayi. Phys. Rev. Lett. 1988. V. 60. P. 956.

[96] M. Flohr. Mod. Phys. Lett. A. 1996. V. 11. P. 55.

[97] J.-C. Lee, X. G. Wen. Electron and quasiparticle exponents of Haldane-Rezayi states in non-abelian fractional quantum Hall theory. cond-mat/9705303.

[98] V. Gurarie, M. Flohr, C. Nayak. Nucl. Phys. B. 1997. V. 498. P. 513.

[99] R. B. Laughlin. Phys. Rev. Lett. 1983. V. 50. P. 1395.

[100] F. Wilczek. Fractional Statistics and Anyon Superconductivity. Singapore: World Scientific, 1990.

[101] G. Moore, N. Seiberg. Nucl. Phys. B. 1989. V. 313. P. 16.

[102] E. Verlinde. Nucl. Phys. B. 1988. V. 300. № 22. P. 360.

[103] R. Dijkgraaf, E. Verlinde, H. Verlinde. Modular invariance and the fusion algebra, Conformal Field Theories and Related Topics. In: Nucl. Phys. B. (Proc. Suppl). Ed. P. Binétruy, P. Sorba, R. Stora. Amsterdam: North-Holland, 1988. V. 5. P. 87.

[104] C. L. Kane, M. Fisher. Phys. Rev. B. 1992. V. 46. P. 15233.

[105] J. Frőhlich, T. Kerler. Nucl. Phys. B. 1991. V. 354. P. 369

[106] J. Frőhlich, E. Thiran. J. Stat. Phys. 1994. V. 76. P. 209.

[107] B. Blok, X. G. Wen. Phys. Rev. B. 1990. V. 42. P. 8133.

[108] N. Read. Phys. Rev. Lett. 1990. V. 65. P. 1502.

[109] S. Lang. Elliptic functions. Reading: Addison-Wesley, 1973.

[110] R. E. Peierls. Quantum Theory of Solids. Oxford: Oxford University Press, 1995.

[111] R. Dijkgraaf. A Geometric Approach to $2 D$ Conformal Field Theory. Ph.D. Thesis. Utrecht: Utrecht University, 1989.

[112] C. Itzykson. Level one Kac-Moody characters and modular invariance, Conformal Field Theories and related topics. In: Nucl. Phys. B. (Proc Suppl.). Ed. P. Binétruy, P. Sorba, R. Stora. Amsterdam: North-Holland, 1988. V. 5. P. 150. 